\title{
The Dirichlet problem in Lipschitz domains with boundary data in Besov spaces for higher order elliptic systems with rough coefficients *
}

\author{
V. Maz'ya, M. Mitrea and T. Shaposhnikova
}

\begin{abstract}
We settle the issue of well-posedness for the Dirichlet problem for a higher order elliptic system $\mathcal{L}\left(x, D_{x}\right)$ with complex-valued, bounded, measurable coefficients in a Lipschitz domain $\Omega$, with boundary data in Besov spaces.

The main hypothesis under which our principal result is established is in the nature of best possible and requires that, at small scales, the mean oscillations of the unit normal to $\partial \Omega$ and of the coefficients of the differential operator $\mathcal{L}\left(x, D_{x}\right)$ are not too large.
\end{abstract}

\section{Introduction}

A fundamental theme in the theory of partial differential equations, which has profound and intriguing connections with many other subareas of analysis, is the well-posedness of various classes of boundary value problems under sharp smoothness assumptions on the boundary of the domain and on the coefficients of the corresponding differential operator. In this paper we initiate a program broadly aimed at extending the scope of the agenda set forth by Agmon, Douglis, Nirenberg and Solonnikov (cf. [4], [45], [46]) in connection with general elliptic boundary value problems on Sobolev-Besov scales, as to allow minimal smoothness assumptions (on the underlying domain and on the coefficients of the differential operator). Our main result is the solvability of the Dirichlet problem for general higher order elliptic systems in divergence form, with complex-valued, bounded, measurable coefficients in Lipschitz domains, and for boundary data in Besov spaces. In order to be more specific we need to introduce some notation.

Let $m, l \in \mathbb{N}$ be two fixed integers and, for a bounded Lipschitz domain $\Omega$ in $\mathbb{R}^{n}$ (a formal definition is given in $\S 6.1)$ with outward unit normal $\nu=\left(\nu_{1}, \ldots, \nu_{n}\right)$ consider the Dirichlet problem for the operator

$$
\mathcal{L}\left(X, D_{X}\right) \mathcal{U}:=\sum_{|\alpha|=|\beta|=m} D^{\alpha}\left(A_{\alpha \beta}(X) D^{\beta} \mathcal{U}\right)
$$

i.e.,

*2000 Math Subject Classification. Primary: 35G15, 35J55, 35J40 Secondary 35J67, 35E05, 46 E39.

Key words: higher order elliptic systems, Besov spaces, weighted Sobolev spaces, mean oscillations, BMO, VMO, Lipschitz domains, Dirichlet problem

The work of authors was supported in part by from NSF DMS and FRG grants as well as from the Swedish National Science Research Council 


$$
\left\{\begin{array}{l}
\sum_{|\alpha|=|\beta|=m} D^{\alpha}\left(A_{\alpha \beta}(X) D^{\beta} \mathcal{U}\right)=0 \quad \text { for } X \in \Omega, \\
\frac{\partial^{k} \mathcal{U}}{\partial \nu^{k}}=g_{k} \quad \text { on } \partial \Omega, \quad 0 \leq k \leq m-1 .
\end{array}\right.
$$

Here and elsewhere, $D^{\alpha}=\left(-i \partial / \partial x_{1}\right)^{\alpha_{1}} \ldots\left(-i \partial / \partial x_{n}\right)^{\alpha_{n}}$ if $\alpha=\left(\alpha_{1}, \ldots, \alpha_{n}\right)$. The coefficients $A_{\alpha \beta}$ are $l \times l$ matrix-valued functions with complex entries satisfying

$$
\sum_{|\alpha|=|\beta|=m}\left\|A_{\alpha \beta}\right\|_{L_{\infty}(\Omega)} \leq \kappa_{1}
$$

for some finite constant $\kappa_{1}$, and such that the coercivity condition

$$
\Re \int_{\Omega} \sum_{|\alpha|=|\beta|=m}\left\langle A_{\alpha \beta}(X) D^{\beta} \mathcal{U}(X), D^{\alpha} \mathcal{U}(X)\right\rangle d X \geq \kappa_{0} \sum_{|\alpha|=m}\left\|D^{\alpha} \mathcal{U}\right\|_{L_{2}(\Omega)}^{2}
$$

with $\kappa_{0}=$ const $>0$ holds for all $\mathbb{C}^{l}$-valued functions $\mathcal{U} \in C_{0}^{\infty}(\Omega)$. Throughout the paper, $\Re z$ denotes the real part of $z \in \mathbb{C}$ and $\langle\cdot, \cdot\rangle$ stands for the canonical inner product in $\mathbb{C}^{l}$.

Since, generally speaking, $\nu$ is merely bounded and measurable, care should be exercised when defining iterated normal derivatives. For the setting we have in mind it is natural to take $\partial^{k} / \partial \nu^{k}:=$ $\left.\left(\sum_{j=1}^{n} \xi_{j} \partial / \partial x_{j}\right)^{k}\right|_{\xi=\nu}$ or, more precisely,

$$
\frac{\partial^{k} \mathcal{U}}{\partial \nu^{k}}:=i^{k} \sum_{|\alpha|=k} \frac{k !}{\alpha !} \nu^{\alpha} \operatorname{Tr}\left[D^{\alpha} \mathcal{U}\right], \quad 0 \leq k \leq m-1
$$

where $\mathrm{Tr}$ is the boundary trace operator and $\nu^{\alpha}:=\nu_{1}^{\alpha_{1}} \cdots \nu_{n}^{\alpha_{n}}$ if $\alpha=\left(\alpha_{1}, \ldots, \alpha_{n}\right)$. With $\rho(X):=$ $\operatorname{dist}(X, \partial \Omega)$ and $p \in(1, \infty), a \in(-1 / p, 1-1 / p)$ fixed, a solution for (1.2) is sought in $W_{p}^{m, a}(\Omega)$, defined as the space of vector-valued functions for which

$$
\left(\sum_{0 \leq|\alpha| \leq m} \int_{\Omega}\left|D^{\alpha} \mathcal{U}(X)\right|^{p} \rho(X)^{p a} d X\right)^{1 / p}<\infty .
$$

In particular, as explained later on, the traces in (1.5) exist in the Besov space $B_{p}^{s}(\partial \Omega)$, where $s:=1-a-1 / p \in(0,1)$, for any $\mathcal{U} \in W_{p}^{m, a}(\Omega)$. Recall that, with $d \sigma$ denoting the area element on $\partial \Omega$

$$
g \in B_{p}^{s}(\partial \Omega) \Leftrightarrow\|g\|_{B_{p}^{s}(\partial \Omega)}:=\|f\|_{L_{p}(\partial \Omega)}+\left(\int_{\partial \Omega} \int_{\partial \Omega} \frac{|g(X)-g(Y)|^{p}}{|X-Y|^{n-1+s p}} d \sigma_{X} d \sigma_{Y}\right)^{1 / p}<\infty
$$

The above definition takes advantage of the Lipschitz manifold structure of $\partial \Omega$. On such manifolds, smoothness spaces of index $s \in(0,1)$ can be defined in an intrinsic, invariant fashion by lifting their Euclidean counterparts onto the manifold itself via local charts. We shall, nonetheless, find it useful to consider higher order smoothness spaces on $\partial \Omega$ in which case the above approach is no longer effective. An alternative point of view has been developed by $\mathrm{H}$. Whitney in [52] where he considered what amounts to higher order Lipschitz spaces on arbitrary closed sets. A far-reaching extension of this circle of ideas pertaining to the full scale of Besov and Sobolev spaces on irregular subsets of $\mathbb{R}^{n}$ can be found in the book [26] by A. Jonsson and H. Wallin. 
For the purpose of this introduction we note that one possible description of these higher order Besov spaces on the boundary of a Lipschitz domain $\Omega \subset \mathbb{R}^{n}$ and for $m \in \mathbb{N}, p \in(1, \infty), s \in(0,1)$, reads

$$
\dot{B}_{p}^{m-1+s}(\partial \Omega)=\text { the closure of }\left\{\left(\left.D^{\alpha} \mathcal{V}\right|_{\partial \Omega}\right)_{|\alpha| \leq m-1}: \mathcal{V} \in C_{0}^{\infty}\left(\mathbb{R}^{n}\right)\right\} \text { in } B_{p}^{s}(\partial \Omega)
$$

(we shall often make no notational distinction between a Banach space $\mathfrak{X}$ and $\mathfrak{X}^{N}=\mathfrak{X} \oplus \cdots \oplus \mathfrak{X}$ for a finite, positive integer $N$ ). A formal definition along with other equivalent characterizations of $\dot{B}_{p}^{m-1+s}(\partial \Omega)$ can be found in $\S 6.4$.

Given (1.5)-(1.6), a necessary condition for the boundary data $\left\{g_{k}\right\}_{0 \leq k \leq m-1}$ in (1.2) is that

$$
\begin{aligned}
& \text { there exists } \dot{f}=\left\{f_{\alpha}\right\}_{|\alpha| \leq m-1} \in \dot{B}_{p}^{m-1+s}(\partial \Omega) \text { such that } \\
& g_{k}=i^{k} \sum_{|\alpha|=k} \frac{k !}{\alpha !} \nu^{\alpha} f_{\alpha}, \quad \text { for each } 0 \leq k \leq m-1 .
\end{aligned}
$$

To state the (analytical and geometrical) conditions under which the problem (1.2), formulated as above, is well-posed, we need one final piece of terminology. By the infinitesimal mean oscillation of a function $F \in L_{1}(\Omega)$ we shall understand the quantity

$$
\{F\}_{\operatorname{Osc}(\Omega)}:=\limsup _{\varepsilon \rightarrow 0}\left(\sup _{\left\{B_{\varepsilon}\right\}_{\Omega}} f_{B_{\varepsilon} \cap \Omega} f_{B_{\varepsilon} \cap \Omega}|F(X)-F(Y)| d X d Y\right),
$$

where $\left\{B_{\varepsilon}\right\}_{\Omega}$ stands for the set of arbitrary balls centered at points of $\Omega$ and of radius $\varepsilon$, and the barred integral is the mean value. In a similar fashion, the infinitesimal mean oscillation of a function $f \in L_{1}(\partial \Omega)$ is defined by

$$
\{f\}_{\mathrm{Osc}(\partial \Omega)}:=\limsup _{\varepsilon \rightarrow 0}\left(\sup _{\left\{B_{\varepsilon}\right\}_{\partial \Omega}} f_{B_{\varepsilon} \cap \partial \Omega} f_{B_{\varepsilon} \cap \partial \Omega}|f(X)-f(Y)| d \sigma_{X} d \sigma_{Y}\right),
$$

where $\left\{B_{\varepsilon}\right\}_{\partial \Omega}$ is the collection of $n$-dimensional balls with centers on $\partial \Omega$ and of radius $\varepsilon$.

Our main result reads as follows; see also Theorem 6.10 for a more general version.

Theorem 1.1 In the above setting, for each $p \in(1, \infty)$ and $s \in(0,1)$, the problem (1.2) with boundary data as in (1.9) has a unique solution $\mathcal{U}$ for which (1.6) holds with $a=1-s-1 / p$ provided the coefficient matrices $A_{\alpha \beta}$ and the exterior normal vector $\nu$ to $\partial \Omega$ satisfy

$$
\{\nu\}_{\operatorname{Osc}(\partial \Omega)}+\sum_{|\alpha|=|\beta|=m}\left\{A_{\alpha \beta}\right\}_{\operatorname{Osc}(\Omega)} \leq C s(1-s)\left(p p^{\prime}+s^{-1}(1-s)^{-1}\right)^{-1}
$$

where $p^{\prime}=p /(p-1)$ is the conjugate exponent of $p$. Above, $C$ is a sufficiently small constant which depends on $\kappa_{0}, \kappa_{1}$ and the Lipschitz constant of $\Omega$, and is independent of $p$ and $s$. Furthermore, the bound (1.12) can be improved for second order operators, i.e. when $m=1$, when the factor $s(1-s)$ in (1.12) can be removed.

Let BMO and VMO stand, respectively, for the John-Nirenberg space of functions of bounded mean oscillations and the Sarason space of functions of vanishing mean oscillations (considered either on $\Omega$ or on $\partial \Omega$ ). Since for an arbitrary function $F$ we have (with the dependence on the 
domain dropped) $\{F\}_{\text {Osc }} \leq 2$ dist $(F, \mathrm{VMO})$ where the distance is taken in BMO, the smallness condition (1.12) in Theorem 1.1 is satisfied if

$$
\operatorname{dist}(\nu, \operatorname{VMO}(\partial \Omega))+\sum_{|\alpha|=|\beta|=m} \operatorname{dist}\left(A_{\alpha \beta}, \operatorname{VMO}(\Omega)\right) \leq C s(1-s)\left(p p^{\prime}+s^{-1}(1-s)^{-1}\right)^{-1} .
$$

In particular, this is trivially the case when $\nu \in \operatorname{VMO}(\partial \Omega)$ and the $A_{\alpha \beta}$ 's belong to $\operatorname{VMO}(\Omega)$, irrespective of $p, s, \kappa_{0}, \kappa_{1}$ and the Lipschitz constant of $\Omega$.

While the Lipschitz character of a domain $\Omega$ controls the infinitesimal mean oscillation of its unit normal, the inequality in the opposite direction is false in general, as seen by considering $\Omega:=\left\{(x, y) \in \mathbb{R}^{2}: y>\varphi_{\varepsilon}(x)\right\}$ with $\varphi_{\varepsilon}(x):=x \sin \left(\varepsilon \log |x|^{-1}\right)$. Indeed, a simple calculation gives $\left\|\varphi_{\varepsilon}^{\prime}\right\|_{\mathrm{BMO}(\mathbb{R})} \leq C \varepsilon$, yet $\left\|\varphi_{\varepsilon}^{\prime}\right\|_{L_{\infty}(\mathbb{R})} \sim 1$ uniformly for $\varepsilon \in(0,1 / 2)$.

An essentially equivalent reformulation of (1.2) is

$$
\left\{\begin{array}{l}
\sum_{|\alpha|=|\beta|=m} D^{\alpha}\left(A_{\alpha \beta}(X) D^{\beta} \mathcal{U}\right)=0 \quad \text { in } \Omega, \\
\operatorname{Tr}\left[D^{\gamma} \mathcal{U}\right]=g_{\gamma} \quad \text { on } \partial \Omega, \quad|\gamma| \leq m-1,
\end{array}\right.
$$

where $\mathcal{U}$ satisfies (1.6) and

$$
\dot{g}:=\left\{g_{\gamma}\right\}_{|\gamma| \leq m-1} \in \dot{B}_{p}^{m-1+s}(\partial \Omega),
$$

though an advantage of the classical formulation (1.2) is that the number of the data is minimal. For a domain $\Omega \subset \mathbb{R}^{2}$ of class $C^{r}, r>\frac{1}{2}$, and for constant coefficient operators, the Dirichlet problem (1.14) has been considered by S. Agmon in [2] where he proved that there exists a unique solution $\mathcal{U} \in C^{m-1+s}(\bar{\Omega}), 0<s<r$, whenever $g_{\gamma}=\left.D^{\gamma} \mathcal{V}\right|_{\partial \Omega},|\gamma| \leq m-1$, for some function $\mathcal{V} \in C^{m-1+s}(\bar{\Omega})$. See also [3] for a related version.

The innovation that allows us to consider, for the first time, boundary data in Besov spaces as in (1.9) and (1.15), is the systematic use of weighted Sobolev spaces such as those associated with the norm in (1.6). In relation to the standard Besov scale in $\mathbb{R}^{n}$, we would like to point out that, thanks to Theorem 4.1 in [25] on the one hand, and Theorem 1.4.2.4 and Theorem 1.4.4.4 in [20] on the other, we have

$$
\begin{aligned}
& a=1-s-\frac{1}{p} \in(0,1-1 / p) \Longrightarrow W_{p}^{m, a}(\Omega) \hookrightarrow B_{p}^{m-1+s+1 / p}(\Omega), \\
& a=1-s-\frac{1}{p} \in(-1 / p, 0) \Longrightarrow B_{p}^{m-1+s+1 / p}(\Omega) \hookrightarrow W_{p}^{m, a}(\Omega) .
\end{aligned}
$$

Of course, $W_{p}^{m, a}(\Omega)$ is just a classical Sobolev space when $a=0$.

Remarkably, the classical trace theory for unweighted Sobolev spaces turns out to have a most satisfactory analogue in this weighted context; for the upper half-space this has been worked out by S.V. Uspenskil in [49], a paper preceded by the significant work of E. Gagliardo in [18] in the unweighted case. As a consequence, we note that under the assumptions made in Theorem 1.1 .

$$
\sum_{|\alpha| \leq m-1}\left\|\operatorname{Tr}\left[D^{\alpha} \mathcal{U}\right]\right\|_{B_{p}^{s}(\partial \Omega)} \sim\left(\sum_{0 \leq|\alpha| \leq m} \int_{\Omega} \rho(X)^{p(1-s)-1}\left|D^{\alpha} \mathcal{U}(X)\right|^{p} d X\right)^{1 / p}
$$


uniformly in $\mathcal{U}$ satisfying $\mathcal{L}\left(X, D_{X}\right) \mathcal{U}=0$ in $\Omega$. The estimate (1.17) can be viewed as a far-reaching generalization of a well-known characterization of the membership of a function to a Besov space in $\mathbb{R}^{n-1}$ in terms of weighted Sobolev norm estimates for its harmonic extension to $\mathbb{R}_{+}^{n}$ (see, e.g., Proposition $7^{\prime}$ on p. 151 of [47]).

Theorem 1.1 is new even in the case when $m=1$ and $A_{\alpha \beta} \in \mathbb{C}^{l \times l}$ (i.e., for second order, constant coefficient systems) and provides a complete answer to the issue of well-posedness of the problem (1.2), (1.9), (1.6) in the sense that the small mean oscillation condition, depending on $p$ and $s$, is in the nature of best possible if one insists of allowing arbitrary indices $p$ and $s$ in (1.9). This can be seen by considering the following Dirichlet problem for the Laplacian in a domain $\Omega \subset \mathbb{R}^{n}$ :

$$
\left\{\begin{array}{l}
\Delta \mathcal{U}=0 \text { in } \Omega, \\
\operatorname{Tr} \mathcal{U}=g \in B_{p}^{s}(\partial \Omega), \\
D^{\alpha} \mathcal{U} \in L_{p}\left(\Omega, \rho(X)^{p(1-s)-1} d X\right), \quad \forall \alpha:|\alpha| \leq 1 .
\end{array}\right.
$$

It has long been known that, already in the case when $\partial \Omega$ exhibits one cone-like singularity, the well-posedness of (1.18) prevents the indices $(s, 1 / p)$ from taking arbitrary values in $(0,1) \times(0,1)$. At a more sophisticated level, the work of D. Jerison and C. Kenig in [25] shows that (1.18) is well-posed in an arbitrary, given Lipschitz domain $\Omega$ if and only if the point $(s, 1 / p)$ belongs to a certain open region $\mathcal{R}_{\Omega} \subseteq(0,1) \times(0,1)$, determined exclusively by the geometry of the domain $\Omega$ (cf. [25] for more details). Let us also mention here that, even when $\partial \Omega$ is smooth and $m=l=1$, a well-known example due to N. Meyers (cf. [35]) shows that the well-posedness of (1.2) in the class of operators with bounded, measurable coefficients confines $p$ to a small neighborhood of 2 .

Broadly speaking, there are two types of questions pertaining to the well-posedness of the Dirichlet problem in a Lipschitz domain $\Omega$ for a divergence form, elliptic system (1.1) of order $2 m$ with boundary data in Besov spaces.

Question I. Granted that the coefficients of $\mathcal{L}$ exhibit a certain amount of smoothness, identifying the Besov spaces for which this boundary value problem is well-posed.

Question II. Alternatively, for a given Besov space characterize the class of Lipschitz domains $\Omega$ and elliptic operators $\mathcal{L}$ for which the aforementioned boundary value problem is well-posed.

These, as well as other related issues, have been a driving force behind many exciting, recent developments in partial differential equations and allied fields. Ample evidence of their impact can be found in C. Kenig's excellent account [27] which describes the state of the art in this field of research up to mid 1990's, with a particular emphasis on the role played by harmonic analysis techniques.

One generic problem which falls under the scope of Question I is to determine the optimal scale of spaces on which the Dirichlet problem for an elliptic system of order $2 m$ is solvable in an arbitrary Lipschitz domain $\Omega$ in $\mathbb{R}^{n}$. The most basic case, that of the constant coefficient Laplacian in arbitrary Lipschitz domains in $\mathbb{R}^{n}$, is now well-understood thanks to the work of B. Dahlberg and C. Kenig [14], in the case of $L_{p}$-data, and D. Jerison and C. Kenig [25], in the case of Besov data. The case of (1.18) for boundary data exhibiting higher regularity (i.e., $s>1$ ) has been recently dealt with by V. Maz'ya and T. Shaposhnikova in [34] where nearly optimal smoothness conditions for $\partial \Omega$ are found in terms of the properties of $\nu$ as a Sobolev space multiplier. Generalizations of (1.18) to the case of variable-coefficient, single, second order elliptic equations have been obtained by M. Mitrea and M. Taylor in [38], 39], [40]. 
In spite of substantial progress in recent years, there remain many basic open questions, particularly for $l>1$ and/or $m>1$ (corresponding to genuine systems and/or higher order equations), even in the case of constant coefficient operators in Lipschitz domains. In this context, one significant problem (as mentioned in, e.g., [16]) is to determine the sharp range of $p$ 's for which the Dirichlet problem for elliptic systems with $L_{p}$-boundary data is well-posed. In [42], J. Pipher and G. Verchota have developed a $L_{p}$-theory for real, constant coefficient, higher order systems $L=\sum_{|\alpha|=2 m} A_{\alpha} D^{\alpha}$ when $p$ is near 2, i.e. $2-\varepsilon<p<2+\varepsilon$ with $\varepsilon>0$ depending on the Lipschitz character of $\Omega$ but this range is not optimal. Recently, more progress for the biharmonic equation and for general constant coefficient, second order systems with real coefficients, which are elliptic in the sense of Legendre-Hadamard was made by Z. Shen in [44], where he further extended the range of $p$ 's from $(2-\varepsilon, 2+\varepsilon)$ to $\left(2-\varepsilon, \frac{2(n-1)}{n-3}+\varepsilon\right)$ for a general Lipschitz domain $\Omega$ in $\mathbb{R}^{n}, n \geq 4$, where as before $\varepsilon=\varepsilon(\partial \Omega)>0$. Let us also mention here the work [1] of V. Adolfsson and J. Pipher who have dealt with the Dirichlet problem for the biharmonic operator in arbitrary Lipschitz domains and with data in Besov spaces, [51] where G. Verchota formulates and solves a Neumann-type problem for the bi-Laplacian in Lipschitz domains and with boundary data in $L_{2}$, [37] where the authors treat the Dirichlet problem for variable coefficient symmetric, real, elliptic systems of second order in an arbitrary Lipschitz domain $\Omega$ and with boundary data in $B_{p}^{s}(\partial \Omega)$, when $2-\varepsilon<p<2+\varepsilon$ and $0<s<1$, as well as the paper [28] by V. Kozlov and V. Maz'ya, which contains an explicit description of the asymptotic behavior of null-solutions of constant coefficient, higher order, elliptic operators near points on the boundary of a domain with a sufficiently small Lipschitz constant.

A successful strategy for dealing with Question II consists of formulating and solving the analogue of the original problem in a standard case, typically when $\Omega=\mathbb{R}_{+}^{n}$ and $\mathcal{L}$ has constant coefficients, and then deviating from this most standard setting by allowing perturbations of a certain magnitude. A paradigm result in this regard, going back to the work of Agmon, Douglis, Nirenberg and Solonnikov in the 50's and 60's is that the Dirichlet problem is solvable in the context of Sobolev-Besov spaces if $\partial \Omega$ is sufficiently smooth and if $\mathcal{L}$ has continuous coefficients. The latter requirement is an artifact of the method of proof (based on Korn's trick of freezing the coefficients) which requires measuring the size of the oscillations of the coefficients in a pointwise sense (as opposed to integral sense, as in (1.10) ). For a version of Question II, corresponding to boundary data of higher regularity, optimal results have been obtained by V. Maz'ya and T. Shaposhnikova in [32. In this context, the natural language for describing the smoothness of the domain $\Omega$ is that of Sobolev space multipliers.

While the study of boundary value problems in a domain $\Omega \subset \mathbb{R}^{n}$ for elliptic differential operators with discontinuous coefficients goes a long way back (for instance, C. Miranda has considered in [36] operators with coefficients in the Sobolev space $W_{n}^{1}$ ), a lot of attention has been devoted lately to the class of operators with coefficients in VMO (it is worth pointing out here that $W_{n}^{1} \hookrightarrow$ VMO on Lipschitz subdomains of $\mathbb{R}^{n}$ ). Much of the impetus for the recent surge of interest in this particular line of work stems from an observation made by F. Chiarenza, M. Frasca and P. Longo in the early 1990's. More specifically, while investigating interior estimates for the solution of a scalar, second-order elliptic differential equation of the form $\mathcal{L U}=F$, these authors have noticed in [10] that $\mathcal{U}$ can be related to $F$ via a potential theoretic representation formula in which the residual terms are commutators between operators of Calderón-Zygmund type, on the one hand, and operators of multiplication by the coefficients of $\mathcal{L}$, on the other hand. This made it possible to control these terms by invoking the commutator estimate of Coifman-Rochberg-Weiss ([12]). Various partial extensions of this result can be found in [5], [6], [7], [1], [19], [21], [43], and the references therein. Here we would just like to mention that, in the whole Euclidean space, a different approach (based on estimates for the Riesz transforms) has been devised by T. Iwaniec 
and C. Sbordone in [24].

Compared to the aforementioned works, our approach is more akin to that of F. Chiarenza and collaborators $([\mathbf{1 0}],[\mathbf{1 1}])$, though there are fundamental differences between solving boundary problems for higher order and for second order operators. One difficulty inherently linked with the case $m>1$ arises from the way the norm in (1.6) behaves under a change of variables $\varkappa: \Omega=$ $\left\{\left(X^{\prime}, X_{n}\right): X_{n}>\varphi\left(X^{\prime}\right)\right\} \rightarrow \mathbb{R}_{+}^{n}$ designed to flatten the Lipschitz surface $\partial \Omega$. When $m=1$, a simple bi-Lipschitz changes of variables such as the inverse of the map $\mathbb{R}_{+}^{n} \ni\left(X^{\prime}, X_{n}\right) \mapsto\left(X^{\prime}, \varphi\left(X^{\prime}\right)+X_{n}\right) \in$ $\Omega$ will do, but matters are considerable more subtle in the case $m>1$. In this latter situation, we employ a special global flattening map first introduced by J. Nečas (in a different context; cf. p. 188 in [41]) and then independently rediscovered and/or further adapted to new settings by several authors, including V. Maz'ya and T. Shaposhnikova in [32], B. Dahlberg, C. Kenig J. Pipher, E. Stein and G. Verchota (cf. [13] and the discussion in [15]), and S. Hofmann and J. Lewis in [22]. Our main novel contribution in this regard is adapting this circle of ideas to the context when one seeks pointwise estimates for higher order derivatives of $\varkappa$ and $\lambda:=\varkappa^{-1}$ in terms of $[\nabla \varphi]_{\mathrm{BMO}\left(\mathbb{R}^{n-1}\right)}$.

Another ingredient of independent interest is deriving estimates for $D_{x}^{\alpha} D_{y}^{\beta} G(x, y)$ where $G$ is the Green function associated with a constant (complex) coefficient system $L(D)$ of order $2 m$ in the upper half space, which are sufficiently well-suited for deriving commutator estimates in the spirit of [12]. The methods employed in earlier work are largely based on explicit representation formulas for $G(x, y)$ and, hence, cannot be adapted easily to the case of non-symmetric, complex coefficient, higher order systems. By way of contrast, our approach consists of proving directly that the residual part $R(x, y):=G(x, y)-\Phi(x-y)$, where $\Phi$ is a fundamental solution for $L(D)$, has the property that $D_{x}^{\alpha} D_{y}^{\beta} R(x, y)$ is a Hardy-type kernel whenever $|\alpha|=|\beta|=m$.

The layout of the paper is as follows. Section 2 contains estimates for the Green function in the upper-half space. Section 3 deals with integral operators (of Calderón-Zygmund and Hardy type) as well as commutator estimates on weighted Lebesgue spaces. In the last part of this section we also revisit Gagliardo's extension operator and establish estimates in the context of BMO. Section 4 contains a discussion of the Dirichlet problem for higher order, variable coefficient elliptic systems in the upper-half space. Then the adjustments necessary to treat the case of an unbounded domain lying above the graph of a Lipschitz function are presented in Section 5. Finally, in Section 6, we explain how to handle the case of a bounded Lipschitz domain, and state and prove Theorem 6.10 (from which Theorem 1.1 follows). This section also contains further complements and extensions of our main result.

\section{Green's matrix estimates in the half-space}

In this section we prove a key estimate for derivatives of Green's matrix associated with the Dirichlet problem for homogeneous, higher-order constant coefficient elliptic systems in the half-space $\mathbb{R}_{+}^{n}$.

\subsection{Statement of the main result}

Let $L\left(D_{x}\right)$ be a matrix-valued differential operator

$$
L\left(D_{x}\right)=\sum_{|\alpha|=2 m} A_{\alpha} D_{x}^{\alpha}
$$


where the $A_{\alpha}$ 's are constant $l \times l$ matrices with complex entries. Throughout the paper, $D_{x}^{\alpha}:=$ $i^{-|\alpha|} \partial_{x_{1}}^{\alpha_{1}} \partial_{x_{2}}^{\alpha_{2}} \cdots \partial_{x_{n}}^{\alpha_{n}}$ if $\alpha=\left(\alpha_{1}, \alpha_{2}, \ldots, \alpha_{n}\right) \in \mathbb{N}_{0}^{n}$. Here and elsewhere, $\mathbb{N}$ stands for the collection of all positive integers and $\mathbb{N}_{0}:=\mathbb{N} \cup\{0\}$.

We assume that $L$ is strongly elliptic, i.e. there exists $\kappa>0$ such that $\sum_{|\alpha|=m}\left\|A_{\alpha}\right\|_{\mathbb{C}^{l \times l}} \leq \kappa^{-1}$ and

$$
\Re\langle L(\xi) \eta, \eta\rangle_{\mathbb{C}^{l}} \geq \kappa|\xi|^{2 m}\|\eta\|_{\mathbb{C}^{l}}^{2}, \quad \forall \xi \in \mathbb{R}^{n}, \quad \forall \eta \in \mathbb{C}^{l} .
$$

In what follows, in order to simplify notations, we shall denote the norms in different finitedimensional real Euclidean spaces by $|\cdot|$ irrespective of their dimensions. Also, quite frequently, we shall make no notational distinction between a space of scalar functions, call it $\mathfrak{X}$, and the space of vector-valued functions (of a fixed, finite dimension) whose components are in $\mathfrak{X}$.

We denote by $F(x)$ a fundamental matrix of the operator $L\left(D_{x}\right)$, i.e. a $l \times l$ matrix solution of the system

$$
L\left(D_{x}\right) F(x)=\delta(x) I_{l} \quad \text { in } \mathbb{R}^{n},
$$

where $I_{l}$ is the $l \times l$ identity matrix and $\delta$ is the Dirac function.

Consider the Dirichlet problem

$$
\left\{\begin{array}{lc}
L\left(D_{x}\right) u=f & \text { in } \mathbb{R}_{+}^{n}, \\
\operatorname{Tr}\left[\partial^{j} u / \partial x_{n}^{j}\right]=f_{j} & j=0,1, \ldots, m-1, \quad \text { on } \mathbb{R}^{n-1}
\end{array}\right.
$$

where $\mathbb{R}_{+}^{n}:=\left\{x=\left(x^{\prime}, x_{n}\right): x^{\prime} \in \mathbb{R}^{n-1}, x_{n}>0\right\}$ and $\mathrm{Tr}$ is the boundary trace operator. Hereafter, we shall identify $\partial \mathbb{R}_{+}^{n}$ with $\mathbb{R}^{n-1}$ in a canonical fashion.

For each $y^{\prime} \in \mathbb{R}^{n-1}$ we introduce the Poisson matrices $P_{0}, \ldots, P_{m-1}$ for problem (2.4), i.e. the solutions of the boundary-value problems

$$
\left\{\begin{array}{l}
L\left(D_{x}\right) P_{j}\left(x, y^{\prime}\right)=0 I_{l} \quad \text { on } \mathbb{R}_{+}^{n}, \\
\left(\frac{\partial^{k}}{\partial x_{n}^{k}} P_{j}\right)\left(\left(x^{\prime}, 0\right), y^{\prime}\right)=\delta_{j k} \delta\left(x^{\prime}-y^{\prime}\right) I_{l} \text { for } x^{\prime} \in \mathbb{R}^{n-1}, 0 \leq k \leq m-1,
\end{array}\right.
$$

where $\delta_{j k}$ is the usual Kronecker symbol and $0 \leq j \leq m-1$. The matrix-valued function $P_{j}\left(x, 0^{\prime}\right)$ is positive homogeneous of degree $j+1-n$, i.e.

$$
P_{j}\left(x, 0^{\prime}\right)=|x|^{j+1-n} P_{j}\left(x /|x|, 0^{\prime}\right), \quad x \in \mathbb{R}^{n},
$$

where $0^{\prime}$ denotes the origin of $\mathbb{R}^{n-1}$. The restriction of $P_{j}\left(\cdot, 0^{\prime}\right)$ to the upper half-sphere $S_{+}^{n-1}$ is smooth and vanishes on the equator along with all of its derivatives up to order $m-1$ (see for example, $\S 10.3$ in [30]). Hence,

$$
\left\|P_{j}\left(x, 0^{\prime}\right)\right\|_{\mathbb{C}^{l \times l}} \leq C \frac{x_{n}^{m}}{|x|^{n+m-1-j}}, \quad x \in \mathbb{R}_{+}^{n},
$$

and, consequently,

$$
\left\|P_{j}\left(x, y^{\prime}\right)\right\|_{\mathbb{C}^{l \times l}} \leq C \frac{x_{n}^{m}}{\left|x-\left(y^{\prime}, 0\right)\right|^{n+m-1-j}}, \quad x \in \mathbb{R}_{+}^{n}, \quad y^{\prime} \in \mathbb{R}^{n-1} .
$$

By $G(x, y)$ we shall denote the Green's matrix of the problem (2.4), i.e. the unique solution of the boundary-value problem 


$$
\left\{\begin{array}{l}
L\left(D_{x}\right) G(x, y)=\delta(x-y) I_{l} \quad \text { for } x \in \mathbb{R}^{n}, \\
\left(\frac{\partial^{j}}{\partial x_{n}^{j}} G\right)\left(\left(x^{\prime}, 0\right), y\right)=0 I_{l} \quad \text { for } x^{\prime} \in \mathbb{R}^{n-1}, \quad 0 \leq j \leq m-1,
\end{array}\right.
$$

where $y \in \mathbb{R}_{+}^{n}$ is regarded as a parameter.

We now introduce the matrix

$$
R(x, y):=F(x-y)-G(x, y), \quad x, y \in \mathbb{R}_{+}^{n},
$$

so that, for each fixed $y \in \mathbb{R}_{+}^{n}$,

$$
\begin{cases}L\left(D_{x}\right) R(x, y)=0 & \text { for } x \in \mathbb{R}^{n}, \\ \left(\frac{\partial^{j}}{\partial x_{n}^{j}} R\right)\left(\left(x^{\prime}, 0\right), y\right)=\left(\frac{\partial^{j}}{\partial x_{n}^{j}} F\right)\left(\left(x^{\prime}, 0\right)-y\right) & \text { for } x^{\prime} \in \mathbb{R}^{n-1}, 0 \leq j \leq m-1 .\end{cases}
$$

Our goal is to prove the following result.

Theorem 2.1 For all multi-indices $\alpha, \beta$ of length $m$

$$
\left\|D_{x}^{\alpha} D_{y}^{\beta} R(x, y)\right\|_{\mathbb{C}^{l \times l}} \leq C|x-\bar{y}|^{-n},
$$

for $x, y \in \mathbb{R}_{+}^{n}$, where $\bar{y}:=\left(y^{\prime},-y_{n}\right)$ is the reflection of the point $y \in \mathbb{R}_{+}^{n}$ with respect to $\partial \mathbb{R}_{+}^{n}$.

In the proof of Theorem 2.1 we distinguish two cases, $n>2 m$ and $n \leq 2 m$, which we shall treat separately. Our argument pertaining to the situation when $n>2 m$ is based on a lemma to be proved in the subsection below.

\subsection{Estimate for a parameter dependent integral}

As a preamble to the proof of Theorem 2.1] here we dispense with the following technical result.

Lemma 2.2 Let $a$ and $b$ be two non-negative numbers and assume that $\zeta \in \mathbb{R}^{N}$. Then for every $\varepsilon>0$ and $0<\delta<N$ there exists a constant $c(N, \varepsilon, \delta)>0$ such that

$$
\int_{\mathbb{R}^{N}} \frac{d \eta}{(|\eta|+a)^{N+\varepsilon}(|\eta-\zeta|+b)^{N-\delta}} \leq \frac{c(N, \varepsilon, \delta)}{a^{\varepsilon}(|\zeta|+a+b)^{N-\delta}}
$$

Proof. Write $\mathcal{J}=\mathcal{J}_{1}+\mathcal{J}_{2}$ where $\mathcal{J}$ stands for the integral in the left side of (2.13) , whereas $\mathcal{J}_{1}$ and $\mathcal{J}_{2}$ denote the integrals obtained by splitting the domain of integration in $\mathcal{J}$ into $B_{a}=\{\eta \in$ $\left.\mathbb{R}^{N}:|\eta|<a\right\}$ and $\mathbb{R}^{n} \backslash B_{a}$, respectively. If $|\zeta|<2 a$, then

$$
\mathcal{J}_{1} \leq a^{-N-\varepsilon} \int_{B_{a}} \frac{d \eta}{(|\eta-\zeta|+b)^{N-\delta}} \leq c a^{-N-\varepsilon} \int_{B_{4 a}} \frac{d \xi}{(|\xi|+b)^{N-\delta}}
$$

Hence

$$
\mathcal{J}_{1} \leq\left\{\begin{array}{l}
c a^{-N-\varepsilon} a^{N} / b^{N-\delta} \quad \text { if } a<b \\
c a^{-N-\varepsilon+\delta} \quad \text { if } a>b
\end{array}\right.
$$


so that, in particular,

$$
|\zeta|<2 a \Longrightarrow \mathcal{J}_{1} \leq c a^{-\varepsilon}(|\zeta|+a+b)^{\delta-N}
$$

Let us now assume that $|\zeta|>2 a$. Then

$$
\mathcal{J}_{1} \leq \int_{B_{a}} \frac{d \eta}{(|\eta|+a)^{N+\varepsilon}} \frac{c}{(|\zeta|+b)^{N-\delta}} \leq c a^{-\varepsilon}(|\zeta|+a+b)^{\delta-N}
$$

which is of the right order. As for $\mathcal{J}_{2}$, we write

$$
\mathcal{J}_{2} \leq \int_{\mathbb{R}^{n} \backslash B_{a}} \frac{d \eta}{|\eta|^{N+\varepsilon}(|\eta-\zeta|+b)^{N-\delta}}=\mathcal{J}_{2,1}+\mathcal{J}_{2,2}
$$

where $\mathcal{J}_{2,1}, \mathcal{J}_{2,2}$ are obtained by splitting the domain of integration in the above integral into the set $\{\eta:|\eta|>\max \{a, 2|\zeta|\}\}$ and its complement in $\mathbb{R}^{n} \backslash B_{a}$. We have

$$
\begin{aligned}
\mathcal{J}_{2,1} & \leq \int_{|\eta|>\max \{a, b, 2|\zeta|\}} \frac{d \eta}{|\eta|^{N+\varepsilon}(|\eta|+b)^{N-\delta}}+\int_{b>|\eta|>\max \{a, b, 2|\zeta|\}} \frac{d \eta}{|\eta|^{N+\varepsilon}(|\eta|+b)^{N-\delta}} \\
& \leq c\left(\int_{|\eta|>\max \{a, b, 2|\zeta|\}} \frac{d \eta}{|\eta|^{2 N+\varepsilon-\delta}}+\frac{1}{b^{N-\delta}} \int_{b>|\eta|>\max \{a, b, 2|\zeta|\}} \frac{d \eta}{|\eta|^{N+\varepsilon}}\right) \\
& \leq \frac{c}{(a+b+|\zeta|)^{N+\varepsilon-\delta}}+\frac{c}{a^{\varepsilon}(a+b+|\zeta|)^{N-\delta}} \\
& \leq \frac{c}{a^{\varepsilon}(a+b+|\zeta|)^{N-\delta}} .
\end{aligned}
$$

There remains to estimate the integral

$$
\mathcal{J}_{2,2}=\int_{B_{2|\zeta|} \backslash B_{a}} \frac{d \eta}{|\eta|^{N+\varepsilon}(|\eta-\zeta|+b)^{N-\delta}}=\mathcal{J}_{2,2}^{(1)}+\mathcal{J}_{2,2}^{(2)},
$$

where $\mathcal{J}_{2,2}^{(1)}$ and $\mathcal{J}_{2,2}^{(2)}$ are obtained by splitting the domain of integration in $\mathcal{J}_{2,2}$ into $B_{|\zeta| / 2} \backslash B_{a}$ and its complement (relative to $B_{2|\zeta|} \backslash B_{a}$ ). On the one hand,

$$
\mathcal{J}_{2,2}^{(1)} \leq \frac{c}{(|\zeta|+b)^{N-\delta}} \int_{B_{|\zeta| / 2} \backslash B_{a}} \frac{d \eta}{|\eta|^{N+\varepsilon}} \leq \frac{c}{a^{\varepsilon}(|\zeta|+a+b)^{N-\delta}} .
$$

On the other hand, whenever $|\zeta|>a / 2$, the integral $\mathcal{J}_{2,2}^{(2)}$, which extends over all $\eta$ 's such that $|\eta|>a, 2|\zeta|>|\eta|>|\zeta| / 2$, can be estimated as

$$
\begin{aligned}
\mathcal{J}_{2,2}^{(2)} & \leq \frac{c}{|\zeta|^{N+\varepsilon}} \int_{B_{2|\zeta| \backslash B_{a}}} \frac{d \eta}{(|\eta-\zeta|+b)^{N-\delta}} \leq \frac{c}{|\zeta|^{N+\varepsilon}} \int_{B_{4|\zeta|}} \frac{d \xi}{(|\xi|+b)^{N-\delta}} \\
& \leq \frac{c}{|\zeta|^{N+\varepsilon}}\left(\int_{\substack{|\xi|<4|\zeta| \\
|\xi|<b}} \frac{d \xi}{(|\xi|+b)^{N-\delta}}+\int_{\substack{|\xi|<4|\zeta| \\
|\xi|>b}} \frac{d \xi}{(|\xi|+b)^{N-\delta}}\right) .
\end{aligned}
$$

Consequently, 


$$
\mathcal{J}_{2,2}^{(2)} \leq \frac{c \min \{|\zeta|, b\}^{N}}{|\zeta|^{N+\varepsilon} b^{N-\delta}}
$$

Using $|\zeta|>a / 2$ and the obvious inequality

$$
\min \{|\zeta|, b\}^{N} \max \left\{|\zeta|, b^{N-\delta}\right\} \leq|\zeta|^{N} b^{N-\delta}
$$

we arrive at

$$
\mathcal{J}_{2,2}^{(2)} \leq c a^{-\varepsilon}(|\zeta|+a+b)^{\delta-N}
$$

The estimate (2.24), along with (2.21) and (2.20), gives the upper bound $c a^{-\varepsilon}(|\zeta|+a+b)^{\delta-N}$ for $\mathcal{J}_{2,2}$. Combining this with (2.19) we obtain the same majorant for $\mathcal{J}_{2}$ which, together with a similar result for $\mathcal{J}_{1}$ already obtained leads to (2.13). The proof of the lemma is therefore complete.

\subsection{Proof of Theorem 2.1 for $n>2 m$}

In the case when $n>2 m$ there exists a unique fundamental matrix $F(x)$ for the the operator (2.1) which is positive homogeneous of degree $2 m-n$. We shall use the integral representation formula

$$
R(x, y)=R_{0}(x, y)+\ldots+R_{m-1}(x, y), \quad x, y \in \mathbb{R}_{+}^{n},
$$

where $R(x, y)$ has been introduced in (2.10) and, with $P_{j}$ as in (2.5), we set

$$
R_{j}(x, y):=\int_{\mathbb{R}^{n-1}} P_{j}\left(x, \xi^{\prime}\right)\left(\frac{\partial^{j}}{\partial x_{n}^{j}} F\right)\left(\left(\xi^{\prime}, 0\right)-y\right) d \xi^{\prime}, \quad 0 \leq j \leq m-1 .
$$

Then, thanks to (2.7) we have

$$
\left\|R_{j}(x, y)\right\|_{\mathbb{C}^{l \times l}} \leq C \int_{\mathbb{R}^{n-1}} \frac{x_{n}^{m}}{\left|x-\left(\xi^{\prime}, 0\right)\right|^{n+m-1-j}} \cdot \frac{d \xi^{\prime}}{\left|\left(\xi^{\prime}, 0\right)-y\right|^{n-2 m+j}} .
$$

Next, putting

$$
\begin{aligned}
N=n-1, & a=x_{n}, \\
\varepsilon=m-j, & b=y_{n}, \\
\delta=2 m-j-1, & \zeta=y^{\prime}-x^{\prime},
\end{aligned}
$$

in the formulation of Lemma 2.2, we obtain from (2.27)

$$
\left\|R_{j}(x, y)\right\|_{\mathbb{C}^{l \times l}} \leq \frac{C x_{n}^{j}}{\left(\left|y^{\prime}-x^{\prime}\right|+x_{n}+y_{n}\right)^{n-2 m+j}}, \quad 0 \leq j \leq m-1 .
$$

Summing up over $j=0, \ldots, m-1$ gives, by virtue of (2.25), the estimate

$$
\|R(x, y)\|_{\mathbb{C}^{l \times l}} \leq C|x-\bar{y}|^{2 m-n}, \quad x, y \in \mathbb{R}_{+}^{n} .
$$

In order to obtain pointwise estimates for derivatives of $R(x, y)$, we make use of the following local estimate for a solution of problem (2.4) with $f=0$. Recall that $W_{p}^{s}$ stands for the classical $L_{p}$-based Sobolev space of order $s$. The particle $l o c$ is used to brand the local versions of these (and other similar) spaces. 
Lemma 2.3 [see [4] Let $\zeta$ and $\zeta_{0}$ be functions in $C_{0}^{\infty}\left(\mathbb{R}^{n}\right)$ such that $\zeta_{0}=1$ in a neighborhood of supp $\zeta$. Then the solution $u \in W_{2}^{m}\left(\mathbb{R}_{+}^{n}\right.$, loc $)$ of problem 2.4) with $f=0$ and $\varphi_{j} \in$ $W_{p}^{k+1-j-1 / p}\left(\mathbb{R}^{n-1}\right.$, loc $)$, where $k \geq m$ and $p \in(1, \infty)$, belongs to $W_{p}^{k+1}\left(\mathbb{R}_{+}^{n}\right.$, loc $)$ and satisfies the estimate

$$
\|\zeta u\|_{W_{p}^{k+1}\left(\mathbb{R}_{+}^{n}\right)} \leq C\left(\sum_{j=0}^{m-1}\left\|\zeta_{0} \varphi_{j}\right\|_{W_{p}^{k+1-j-1 / p}\left(\mathbb{R}^{n-1}\right)}+\left\|\zeta_{0} u\right\|_{L_{p}\left(\mathbb{R}_{+}^{n}\right)}\right),
$$

where $C$ is independent of $u$ and $\varphi_{j}$.

Let $B(x, r)$ denote the ball of radius $r>0$ centered at $x$.

Corollary 2.4 Assume that $u \in W_{2}^{m}\left(\mathbb{R}_{+}^{n}\right.$, loc $)$ is a solution of problem (2.4) with $f=0$ and $\varphi_{j} \in C^{k+1-j}\left(\mathbb{R}^{n-1}\right.$, loc $)$. Then for any $z \in \overline{\mathbb{R}_{+}^{n}}$ and $\rho>0$

$$
\sup _{\mathbb{R}_{+}^{n} \cap B(z, \rho)}\left|\nabla_{k} u\right| \leq C\left(\rho^{-k} \sup _{\mathbb{R}_{+}^{n} \cap B(z, 2 \rho)}|u|+\sum_{j=0}^{m-1} \sum_{s=0}^{k+1-j} \rho^{s+j-k} \sup _{\mathbb{R}^{n-1} \cap B(z, 2 \rho)}\left|\nabla_{s}^{\prime} \varphi_{j}\right|\right),
$$

where $\nabla_{s}^{\prime}$ is the gradient of order $s$ in $\mathbb{R}^{n-1}$. Here $C$ is a constant independent of $\rho, z$, $u$ and $f_{j}$.

Proof. Given the dilation invariant nature of the estimate we seek, it suffices to assume that $\rho=1$. Given $\phi \in C^{k+1-j}\left(\mathbb{R}^{n-1}\right)$ supported in $\mathbb{R}^{n-1} \cap B(z, 2)$, we observe that, for a suitable $\theta \in(0,1)$,

$$
\begin{aligned}
\|\phi\|_{W_{p}^{k+1-j-1 / p}\left(\mathbb{R}^{n-1}\right)} & \leq C \quad\|\phi\|_{L_{p}\left(\mathbb{R}^{n-1}\right)}^{\theta}\|\phi\|_{W_{p}^{k+1-j}\left(\mathbb{R}^{n-1}\right)}^{1-\theta} \\
& \leq C \sum_{s=0}^{k+1-j} \sup _{\mathbb{R}^{n-1} \cap B(z, 2)}\left|\nabla_{s}^{\prime} \phi\right| .
\end{aligned}
$$

Also, if $p>n$,

$$
\sup _{\mathbb{R}_{+}^{n}}\left|\nabla_{k} v\right| \leq C\|v\|_{W_{p}^{k+1}\left(\mathbb{R}_{+}^{n}\right)},
$$

by virtue of the classical Sobolev inequality. Combining (2.32), (2.33) with Lemma 2.3 now readily gives (2.31).

Given $x, y \in \mathbb{R}_{+}^{n}$, set $\rho:=|x-\bar{y}| / 5$ and pick $z \in \partial \mathbb{R}_{+}^{n}$ such that $|x-z|=\rho / 2$. It follows that for any $w \in \mathbb{R}_{+}^{n} \cap B(z, 2 \rho)$ we have $|x-\bar{y}| \leq|x-z|+|z-w|+|w-\bar{y}| \leq \rho / 2+2 \rho+|w-\bar{y}| \leq|x-\bar{y}| / 2+|w-\bar{y}|$. Consequently, $|x-\bar{y}| / 2 \leq|w-\bar{y}|$ for every $w \in \mathbb{R}_{+}^{n} \cap B(z, 2 \rho)$, so that, ultimately,

$$
\rho^{\nu-k} \sup _{w \in \mathbb{R}^{n-1} \cap B(z, 2 \rho)}\left\|\nabla_{\nu}^{\prime} F(w-y)\right\|_{\mathbb{C}^{l \times l}} \leq \frac{C}{|x-\bar{y}|^{n-2 m+k}},
$$

for each $\nu \in \mathbb{N}_{0}$. Granted (2.29) and our choice of $\rho$, we altogether obtain that

$$
\left\|D_{x}^{\alpha} R(x, y)\right\|_{\mathbb{C}^{l \times l}} \leq C_{k}|x-\bar{y}|^{2 m-n-k}, \quad x, y \in \mathbb{R}_{+}^{n},
$$

for each multi-index $\alpha \in \mathbb{N}_{0}^{n}$ of length $k$. 
In the following two formulas, it will be convenient to use the notation $R_{\mathcal{L}}$ for the matrix $R$ associated with the operator $\mathcal{L}\left(D_{x}\right)$ as in (2.10). By Green's formula

$$
R_{\mathcal{L}}(y, x)=\left[R_{\mathcal{L}^{*}}(x, y)\right]^{*}, \quad x, y \in \mathbb{R}_{+}^{n},
$$

where the superscript star indicates adjunction.

In order to estimate mixed partial derivatives, we observe that (2.36) entails

$$
\left(D_{y}^{\beta} R_{\mathcal{L}}\right)(x, y)=\left[\left(D_{x}^{\beta} R_{\mathcal{L}^{*}}\right)(y, x)\right]^{*}
$$

and remark that $\mathcal{L}^{*}$ has properties similar to $\mathcal{L}$. This, in concert with (2.35) and

$$
|x-\bar{y}|=|\bar{x}-y|, \quad x, y \in \mathbb{R}_{+}^{n} .
$$

yields

$$
\left\|D_{y}^{\beta} R(x, y)\right\|_{\mathbb{C}^{l \times l}} \leq C_{\beta}|x-\bar{y}|^{2 m-n-|\beta|} .
$$

Let us also point out that by formally differentiating (2.11) with respect to $y$ we obtain

$$
\begin{cases}\mathcal{L}\left(D_{x}\right)\left[D_{y}^{\beta} R_{\mathcal{L}}(x, y)\right]=0 & \text { for } x \in \mathbb{R}^{n}, \\ \left(\frac{\partial^{j}}{\partial x_{n}^{j}} D_{y}^{\beta} R\right)\left(\left(x^{\prime}, 0\right), y\right)=\left(\frac{\partial^{j}}{\partial x_{n}^{j}}(-D)^{\beta} F\right)\left(\left(x^{\prime}, 0\right)-y\right), x^{\prime} \in \mathbb{R}^{n-1}, 0 \leq j \leq m-1 .\end{cases}
$$

With (2.39) and (2.40) in place of (2.29) and (2.11), respectively, we can now run the same program as above and obtain the estimate

$$
\left\|D_{x}^{\alpha} D_{y}^{\beta} R(x, y)\right\|_{\mathbb{C}^{l \times l}} \leq C_{\alpha \beta}|x-\bar{y}|^{2 m-n-|\alpha|-|\beta|},
$$

for all multi-indices $\alpha$ and $\beta$.

\subsection{Proof of Theorem 2.1 for $n \leq 2 m$}

When $n \leq 2 m$ we shall use the method of descent. To get started, fix an integer $N$ such that $N>2 m$ and let $(x, z) \mapsto \mathcal{G}(x, y, z-\zeta)$ denote the Green matrix with singularity at $(y, \zeta) \in \mathbb{R}^{n} \times \mathbb{R}^{N-n}$ of the Dirichlet problem for the operator $\mathcal{L}\left(D_{x}\right)+\left(-\Delta_{z}\right)^{m}$ in the $N$-dimensional half-space

$$
\mathbb{R}_{+}^{N}:=\left\{(x, z): z \in \mathbb{R}^{N-n}, x=\left(x^{\prime}, x_{n}\right), x^{\prime} \in \mathbb{R}^{n-1}, x_{n}>0\right\} .
$$

Also, recall that $G(x, y)$ stands for the Green matrix of the problem (2.4). Our immediate goal is to establish the following.

Lemma 2.5 For all multi-indices $\alpha$ and $\beta$ of order $m$ and for all $x$ and $y$ in $\mathbb{R}_{+}^{n}$

$$
D_{x}^{\alpha} D_{y}^{\beta} G\left(x^{\prime}, y\right)=\int_{\mathbb{R}^{N-n}} D_{x}^{\alpha} D_{y}^{\beta} \mathcal{G}(x, y,-\zeta) d \zeta
$$


Proof. The strategy is to show that

$$
\int_{\mathbb{R}_{+}^{n}} D_{x}^{\alpha} D_{y}^{\beta} G(x, y) f_{\beta}(y) d y=\int_{\mathbb{R}_{+}^{n}} \int_{\mathbb{R}^{N-n}} D_{x}^{\alpha} D_{y}^{\beta} \mathcal{G}(x, y,-\zeta) d \zeta f_{\beta}(y) d y
$$

for each $f_{\beta} \in C_{0}^{\infty}\left(\mathbb{R}_{+}^{n}\right)$, from which (2.43) clearly follows. To justify (2.44) for a fixed, arbitrary $f_{\beta} \in C_{0}^{\infty}\left(\mathbb{R}_{+}^{n}\right)$, we let $u$ be the unique vector-valued function satisfying $D^{\alpha} u \in L^{2}\left(\mathbb{R}_{+}^{n}\right)$ for all $\alpha$ with $|\alpha|=m$, and such that

$$
\left\{\begin{array}{l}
\mathcal{L}\left(D_{x}\right) u=D_{x}^{\beta} f_{\beta} \quad \text { in } \mathbb{R}_{+}^{n}, \\
\left(\frac{\partial^{j} u}{\partial x_{n}^{j}}\right)\left(x^{\prime}, 0\right)=0 \quad \text { on } \mathbb{R}^{n-1}, 0 \leq j \leq m-1 .
\end{array}\right.
$$

It is well-known that for each $\gamma \in \mathbb{N}_{0}^{n}$

$$
\left|D^{\gamma} u(x)\right| \leq C_{\gamma}|x|^{m-n-|\gamma|} \quad \text { for }|x|>1 .
$$

This follows, for instance, from Theorem 6.1.4 [29] combined with Theorem 10.3.2 [30]. Also, as a consequence of Green's formula, the solution of the problem (2.45) satisfies

$$
D_{x}^{\alpha} u(x)=\int_{\mathbb{R}_{+}^{n}} D_{x}^{\alpha}\left(-D_{y}\right)^{\beta} G(x, y) f_{\beta}(y) d y .
$$

We shall now derive yet another integral representation formula for $D_{x}^{\alpha} u$ in terms of (derivatives of) $\mathcal{G}$ which is similar in spirit to (2.47). To get started, we note that since $N>2 m$ the estimate (2.41) implies

$$
\left\|D_{x}^{\alpha} D_{y}^{\beta} \mathcal{G}(x, y,-\zeta)\right\|_{\mathbb{C}^{l \times l}} \leq c(|x-y|+|\zeta|)^{-N} .
$$

Let us now fix $x \in \mathbb{R}_{+}^{n}, \rho>0$ and introduce a cut-off function $H \in C^{\infty}\left(\mathbb{R}^{N-n}\right)$ which satisfies $H(z)=1$ for $|z| \leq 1$ and $H(z)=0$ for $|z| \geq 2$. We may then write

$$
u(x)=\int_{\mathbb{R}^{N}} \mathcal{G}(x, y,-\zeta)\left[H(\zeta / \rho) D^{\beta} f_{\beta}(y)+\left(-\Delta_{\zeta}\right)^{m}(H(\zeta / \rho) u(y))\right] d y d \zeta,
$$

which further implies

$$
\begin{aligned}
& \left|D_{x}^{\alpha} u(x)-\int_{\mathbb{R}^{N}} D_{x}^{\alpha}\left(-D_{y}\right)^{\beta} \mathcal{G}(x, y,-\zeta) H(\zeta / \rho) f_{\beta}(y) d y d \zeta\right| \\
& \quad \leq c \sum_{|\gamma|=m} \int_{\mathbb{R}_{+}^{N}}\left\|D_{x}^{\alpha} D_{\zeta}^{\gamma} \mathcal{G}(x, y,-\zeta)\right\|_{\mathbb{C}^{l \times l}}\left|u(y) D_{\zeta}^{\gamma}(H(\zeta / \rho))\right| d \zeta
\end{aligned}
$$

By (2.46) and (2.48), the expression in the right-hand side of (2.50) does not exceed

$$
\begin{aligned}
& c \rho^{-m} \int_{\rho<|\zeta|<2 \rho} d \zeta \int_{\mathbb{R}^{n-1}}(|x-y|+|\zeta|)^{-N}|y|^{m-n} d y \\
& \quad \leq c \rho^{N-n-m} \int_{\mathbb{R}^{n-1}}(|y|+\rho)^{-N}|y|^{m-n} d y=c \rho^{-n}
\end{aligned}
$$


This estimate, in concert with (2.48), allows us to obtain, after making $\rho \rightarrow \infty$, that

$$
D_{x}^{\alpha} u(x)=\int_{\mathbb{R}_{+}^{n}} \int_{\mathbb{R}^{N-n}} D_{x}^{\alpha}\left(-D_{y}\right)^{\beta} \mathcal{G}(x, y,-\zeta) d \zeta f_{\beta}(y) d y .
$$

Now (2.44) follows readily from this and (2.47).

Having disposed of Lemma 2.5] we are ready to present the

End of Proof of Theorem 2.1. Assume that $2 m \geq n$ and let $N$ be again an integer such that $N>2 m$. Denote by $\mathcal{F}(x, z)$ the fundamental solution of the operator $\mathcal{L}\left(D_{x}\right)+\left(-\Delta_{z}\right)^{m}$, which is positive homogeneous of degree $2 m-N$ and is singular at $(0,0) \in \mathbb{R}^{n} \times \mathbb{R}^{N-n}$. Then the identity

$$
D_{x}^{\alpha+\beta} F(x)=\int_{\mathbb{R}^{N-n}} D_{x}^{\alpha+\beta} \mathcal{F}(x,-\zeta) d \zeta
$$

can be established by proceeding as in the proof of Lemma 2.5. Combining (2.52) with Lemma 2.5 we arrive at

$$
D_{x}^{\alpha} D_{y}^{\beta} R\left(x^{\prime}, y\right)=\int_{\mathbb{R}^{N-n}} D_{x}^{\alpha} D_{y}^{\beta} \mathcal{R}(x, y,-\zeta) d \zeta,
$$

where $\mathcal{R}(x, y, z):=\mathcal{G}(x, y, z)-\mathcal{F}(x-y, z)$. Consequently,

$$
\left\|D_{x}^{\alpha} D_{y}^{\beta} \mathcal{R}(x, y,-\zeta)\right\|_{\mathbb{C}^{l \times l}} \leq C(|x-\bar{y}|+|\zeta|)^{-N}
$$

by (2.35) with $k=0$ and $N$ in place of $n$. This estimate, together with (2.53), yields (2.12) and the proof of Theorem 2.1 is therefore complete.

\section{Properties of integral operators in a half-space}

In $\S 3.1$ and $\S 3.2$ we prove estimates for commutators (and certain commutator-like operators) between integral operators in $\mathbb{R}_{+}^{n}$ and multiplication operators with functions of bounded mean oscillations, in weighted Lebesgue spaces on $\mathbb{R}_{+}^{n}$. Subsection 3.3 contains BMO and pointwise estimates for extension operators from $\mathbb{R}^{n-1}$ onto $\mathbb{R}_{+}^{n}$.

Throughout this section, given two Banach spaces $E, F$, we let $\mathfrak{L}(E, F)$ stand for the space of bounded linear operators from $E$ into $F$, and abbreviate $\mathfrak{L}(E):=\mathfrak{L}(E, E)$. Also, given $p \in[1, \infty]$, an open set $\mathcal{O} \subset \mathbb{R}^{n}$ and a measurable nonnegative function $w$ on $\mathcal{O}$, we let $L_{p}(\mathcal{O}, w(x) d x)$ denote the usual Lebesgue space of (classes of) functions which are $p$-th power integrable with respect to the weighted measure $w(x) d x$ on $\mathcal{O}$. Finally, following a well-established tradition, $A(r) \sim B(r)$ will mean that each quantity is dominated by a fixed multiple of the other, uniformly in the parameter $r$.

\subsection{Kernels with singularity at $\partial \mathbb{R}_{+}^{n}$}

Recall $L_{p}\left(\mathbb{R}_{+}^{n}, x_{n}^{a p} d x\right)$ stands for the weighted Lebesgue space of $p$-th power integrable functions in $\mathbb{R}_{+}^{n}$ corresponding to the weight $w(x):=x_{n}^{a p}, x=\left(x^{\prime}, x_{n}\right) \in \mathbb{R}_{+}^{n}$.

Proposition 3.1 Let $a \in \mathbb{R}, 1<p<\infty$, and assume that $\mathcal{Q}$ is a non-negative measurable function on $\left\{\zeta=\left(\zeta^{\prime}, \zeta_{n}\right) \in \mathbb{R}^{n-1} \times \mathbb{R}: \zeta_{n}>-1\right\}$, which also satisfies 


$$
\int_{\mathbb{R}_{+}^{n}} \mathcal{Q}\left(\zeta^{\prime}, \zeta_{n}-1\right) \zeta_{n}^{-a-1 / p} d \zeta<\infty
$$

Then the operator

$$
Q f(x):=x_{n}^{-n} \int_{\mathbb{R}_{+}^{n}} \mathcal{Q}\left(\frac{y-x}{x_{n}}\right) f(y) d y, \quad x=\left(x^{\prime}, x_{n}\right) \in \mathbb{R}_{+}^{n},
$$

initially defined on functions $f \in L_{p}\left(\mathbb{R}_{+}^{n}\right)$ with compact support in $\mathbb{R}_{+}^{n}$, can be extended by continuity to an operator acting from $L_{p}\left(\mathbb{R}_{+}^{n}, x_{n}^{a p} d x\right)$ into itself, with the norm satisfying

$$
\|Q\|_{\mathcal{L}\left(L_{p}\left(\mathbb{R}_{+}^{n}, x_{n}^{a p} d x\right)\right)} \leq \int_{\mathbb{R}_{+}^{n}} \mathcal{Q}\left(\zeta^{\prime}, \zeta_{n}-1\right) \zeta_{n}^{-a-1 / p} d \zeta .
$$

Proof. Introducing the new variable $\zeta:=\left(x_{n}^{-1}\left(y^{\prime}-x^{\prime}\right), x_{n}^{-1} y_{n}\right) \in \mathbb{R}_{+}^{n}$, we may write

$$
|Q f(x)| \leq \int_{\mathbb{R}_{+}^{n}} \mathcal{Q}\left(\zeta^{\prime}, \zeta_{n}-1\right)\left|f\left(x^{\prime}+x_{n} \zeta^{\prime}, x_{n} \zeta_{n}\right)\right| d \zeta, \quad \forall x \in \mathbb{R}_{+}^{n}
$$

Then, by Minkowski's inequality,

$$
\begin{aligned}
\|Q f\|_{L_{p}\left(\mathbb{R}_{+}^{n}, x_{n}^{a p} d x\right)} & \leq \int_{\mathbb{R}_{+}^{n}} \mathcal{Q}\left(\zeta^{\prime}, \zeta_{n}-1\right)\left(\int_{\mathbb{R}_{+}^{n}} x_{n}^{a p}\left|f\left(x^{\prime}+x_{n} \zeta^{\prime}, x_{n} \zeta_{n}\right)\right|^{p} d x\right)^{1 / p} d \zeta \\
& =\left(\int_{\mathbb{R}_{+}^{n}} \mathcal{Q}\left(\zeta^{\prime}, \zeta_{n}-1\right) \zeta_{n}^{-a-1 / p} d \zeta\right)\|f\|_{L_{p}\left(\mathbb{R}_{+}^{n}, x_{n}^{a p} d x\right)},
\end{aligned}
$$

as desired.

Recall that $\bar{y}:=\left(y^{\prime},-y_{n}\right)$ if $y=\left(y^{\prime}, y_{n}\right) \in \mathbb{R}^{n-1} \times \mathbb{R}$.

Corollary 3.2 Consider

$$
R f(x):=\int_{\mathbb{R}_{+}^{n}} \frac{\log \left(\frac{|x-y|}{x_{n}}+2\right)}{|x-\bar{y}|^{n}} f(y) d y, \quad x=\left(x^{\prime}, x_{n}\right) \in \mathbb{R}_{+}^{n} .
$$

Then for each $1<p<\infty$ and each $a \in(-1 / p, 1-1 / p)$ the operator $R$ is bounded from $L_{p}\left(\mathbb{R}_{+}^{n}, x_{n}^{a p} d x\right)$ into itself. Moreover,

$$
\|R\|_{\mathfrak{L}\left(L_{p}\left(\mathbb{R}_{+}^{n}, x_{n}^{a p} d x\right)\right)} \leq \frac{c(n) p^{2}}{(p a+1)(p(1-a)-1)}=\frac{c(n)}{s(1-s)},
$$

where $s=1-a-1 / p$ and $c(n)$ is independent of $p$ and $a$.

Proof. The result follows from Proposition 3.1] with

$$
\mathcal{Q}(\zeta):=\frac{\log (|\zeta|+2)}{\left(|\zeta|^{2}+1\right)^{n / 2}},
$$

and from the obvious inequality $2|x-\bar{y}|^{2} \geq|x-y|^{2}+x_{n}^{2}$.

Let us note here that Corollary 3.2 immediately yields the following. 
Corollary 3.3 Consider

$$
K f(x):=\int_{\mathbb{R}_{+}^{n}} \frac{f(y)}{|x-\bar{y}|^{n}} d y, \quad x \in \mathbb{R}_{+}^{n} .
$$

Then for each $1<p<\infty$ and $a \in(-1 / p, 1-1 / p)$ the operator $K$ is bounded from $L_{p}\left(\mathbb{R}_{+}^{n}, x_{n}^{a p} d x\right)$ into itself. Moreover,

$$
\|K\|_{\mathfrak{L}\left(L_{p}\left(\mathbb{R}_{+}^{n}, x_{n}^{a p} d x\right)\right)} \leq \frac{c(n) p^{2}}{(p a+1)(p(1-a)-1)}=\frac{c(n)}{s(1-s)}
$$

where $s=1-a-1 / p$ and $c(n)$ is independent of $p$ and $a$.

Recall that the barred integral stands for the mean-value (taken in the integral sense).

Lemma 3.4 Assume that $1<p<\infty, a \in(-1 / p, 1-1 / p)$, and recall the operator $K$ introduced in (3.9). Further, consider a non-negative, measurable function $w$ defined on $\mathbb{R}_{+}^{n}$ and fix a family of balls $\mathcal{F}$ which form a Whitney covering of $\mathbb{R}_{+}^{n}$. Then the norm of $w K$ as an operator from $L_{p}\left(\mathbb{R}_{+}^{n}, x_{n}^{a p} d x\right)$ into itself is equivalent to

$$
\sup _{B \in \mathcal{F}} f_{B} w(y)^{p} d y
$$

Furthermore,

$$
\|w K\|_{\mathfrak{L}\left(L_{p}\left(\mathbb{R}_{+}^{n}, x_{n}^{a p} d x\right)\right)} \leq \frac{c(n)}{s(1-s)} \sup _{B \in \mathcal{F}}\left(f_{B} w(y)^{p} d y\right)^{1 / p}
$$

where $c(n)$ is independent of $w, p$, and $\alpha$.

Proof. Fix $f \geq 0$ and denote by $|B|$ the Euclidean volume of $B$. Sobolev's embedding theorem allows us to write

$$
\|K f\|_{L_{\infty}(B)}^{p} \leq c(n)|B|^{-1} \sum_{j=0}^{n}|B|^{j p / n}\left\|\nabla_{j} K f\right\|_{L_{p}(B)}^{p}, \quad \forall B \in \mathcal{F} .
$$

Hence,

$$
\int_{\mathbb{R}_{+}^{n}}\left|x_{n}^{a} w(x)(K f)(x)\right|^{p} d x \leq c(n) \sup _{B \in \mathcal{F}} f_{B} w(y)^{p} d y \int_{\mathbb{R}_{+}^{n}} x_{n}^{p a} \sum_{0 \leq j \leq l} x_{n}^{j p}\left|\nabla_{j} K f\right|^{p} d x
$$

Observing that $x_{n}^{j}\left|\nabla_{j} K f\right| \leq c(n) K f$ and referring to Corollary 3.3. we arrive at the required upper estimate for the norm of $w K$. The lower estimate is obvious.

We momentarily pause in order to collect some definitions and set up basic notation pertaining to functions with bounded mean oscillations. Let $f$ be a locally integrable function defined on $\mathbb{R}^{n}$ and define the seminorm

$$
[f]_{\mathrm{BMO}\left(\mathbb{R}^{n}\right)}:=\sup _{B} f_{B}\left|f(x)-f_{B} f(y) d y\right| d x
$$


where the supremum is taken over all balls $B$ in $\mathbb{R}^{n}$. Next, if $f$ is a locally integrable function defined on $\mathbb{R}_{+}^{n}$, we set

$$
[f]_{\mathrm{BMO}\left(\mathbb{R}_{+}^{n}\right)}:=\sup _{\{B\}} f_{B \cap \mathbb{R}_{+}^{n}}\left|f(x)-f_{B \cap \mathbb{R}_{+}^{n}} f(y) d y\right| d x,
$$

where, this time, the supremum is taken over the collection $\{B\}$ of all balls $B$ with centers in $\overline{\mathbb{R}_{+}^{n}}$. Then the following inequalities are straightforward

$$
[f]_{\mathrm{BMO}\left(\mathbb{R}_{+}^{n}\right)} \leq \sup _{\{B\}} f_{B \cap \mathbb{R}_{+}^{n}} f_{B \cap \mathbb{R}_{+}^{n}}|f(x)-f(y)| d x d y \leq 2[f]_{\mathrm{BMO}\left(\mathbb{R}_{+}^{n}\right)} .
$$

We also record here the equivalence relation

$$
[f]_{\mathrm{BMO}\left(\mathbb{R}_{+}^{n}\right)} \sim[\operatorname{Ext} f]_{\mathrm{BMO}\left(\mathbb{R}^{n}\right)},
$$

where Ext $f$ is the extension of $f$ onto $\mathbb{R}^{n}$ as an even function in $x_{n}$.

Finally, by $\mathrm{BMO}\left(\mathbb{R}_{+}^{n}\right)$ we denote the collection of equivalence classes, modulo constants, of functions $f$ on $\mathbb{R}_{+}^{n}$ for which $[f]_{\mathrm{BMO}\left(\mathbb{R}_{+}^{n}\right)}<\infty$.

Proposition 3.5 Let $b \in \mathrm{BMO}\left(\mathbb{R}_{+}^{n}\right)$ and consider the operator

$$
T f(x):=\int_{\mathbb{R}_{+}^{n}} \frac{|b(x)-b(y)|}{|x-\bar{y}|^{n}} f(y) d y, \quad x \in \mathbb{R}_{+}^{n} .
$$

Then for each $p \in(1, \infty)$ and $a \in(-1 / p, 1-1 / p)$

$$
T: L_{p}\left(\mathbb{R}_{+}^{n}, x_{n}^{a p} d x\right) \longrightarrow L_{p}\left(\mathbb{R}_{+}^{n}, x_{n}^{a p} d x\right)
$$

is a well-defined, bounded operator with

$$
\|T\|_{\mathfrak{L}\left(L_{p}\left(\mathbb{R}_{+}^{n}, x_{n}^{a p} d x\right)\right)} \leq \frac{c(n)}{s(1-s)}[b]_{\mathrm{BMO}\left(\mathbb{R}_{+}^{n}\right)},
$$

where $c(n)$ is a constant which depends only on $n$.

Proof. Given $x \in \mathbb{R}_{+}^{n}$ and $r>0$, we shall use the abbreviations

$$
\bar{b}_{r}(x):=f_{B(x, r) \cap \mathbb{R}_{+}^{n}} b(y) d y, \quad D_{r}(x):=\left|b(x)-\bar{b}_{r}(x)\right|,
$$

and make use of the integral operator

$$
S f(x):=\int_{\mathbb{R}_{+}^{n}} \frac{D_{|x-\bar{y}|}(x)}{|x-\bar{y}|^{n}} f(y) d y, \quad x \in \mathbb{R}_{+}^{n},
$$

as well as its adjoint $S^{*}$. Clearly, for each nonnegative, measurable function $f$ on $\mathbb{R}_{+}^{n}$ and each $x \in \mathbb{R}_{+}^{n}$,

$$
\begin{aligned}
T f(x) & \leq S f(x)+S^{*} f(x)+\int_{\mathbb{R}_{+}^{n}} \frac{\left|\bar{b}_{|x-\bar{y}|}(x)-\bar{b}_{|x-\bar{y}|}(y)\right|}{|x-\bar{y}|^{n}} f(y) d y \\
& \leq S f(x)+S^{*} f(x)+c(n)[b]_{\mathrm{BMO}\left(\mathbb{R}_{+}^{n}\right)} K f(x)
\end{aligned}
$$


where $K$ has been introduced in (3.9). Making use of Corollary 3.3. we need to estimate only the norm of $S$. Obviously,

$$
S f(x) \leq D_{x_{n}}(x) K f(x)+\int_{\mathbb{R}_{+}^{n}} \frac{\left|\bar{b}_{x_{n}}(x)-\bar{b}_{|x-\bar{y}|}(x)\right|}{|x-\bar{y}|^{n}} f(y) d y .
$$

Setting $r=|x-\bar{y}|$ and $\rho=x_{n}$ in the standard inequality

$$
\left|\bar{b}_{\rho}(x)-\bar{b}_{r}(x)\right| \leq c(n) \log \left(\frac{r}{\rho}+1\right)[b]_{\mathrm{BMO}\left(\mathbb{R}_{+}^{n}\right)},
$$

where $r>\rho$ (cf., e.g., p. 176 in [32], or p. 206 in [48]), we arrive at

$$
S f(x) \leq D_{x_{n}}(x) K f(x)+c(n)[b]_{B M O\left(\mathbb{R}_{+}^{n}\right)} R f(x),
$$

where $R$ is defined in (3.6). Let $\mathcal{F}$ be a Whitney covering of $\mathbb{R}_{+}^{n}$ with open balls. For an arbitrary $B \in \mathcal{F}$, denote by $\delta$ the radius of $B$. By Lemma 3.4 with $w(x):=D_{x_{n}}(x)$, the norm of the operator $D_{x_{n}}(x) K$ does not exceed

$$
\begin{aligned}
\sup _{B \in \mathcal{F}}\left(f_{B}\left|D_{x_{n}}(x)\right|^{p} d x\right)^{1 / p} & \leq c(n) \sup _{B \in \mathcal{F}}\left(f_{B}\left|b(x)-\bar{b}_{\delta}(x)\right|^{p} d x\right)^{1 / p}+c(n)[b]_{\mathrm{BMO}\left(\mathbb{R}_{+}^{n}\right)} \\
& \leq c(n)[b]_{\mathrm{BMO}\left(\mathbb{R}_{+}^{n}\right)},
\end{aligned}
$$

by the John-Nirenberg inequality. Here we have also used the triangle inequality and the estimate (3.26) in order to replace $\bar{b}_{x_{n}}(x)$ in the definition of $D_{x_{n}}(x)$ by $\bar{b}_{\delta}(x)$. The intervening logarithmic factor is bounded independently of $x$ since $x_{n}$ is comparable with $\delta$, uniformly for $x \in B$. With this estimate in hand, a reference to Corollary 3.2 gives that

$$
\begin{aligned}
& S: L_{p}\left(\mathbb{R}_{+}^{n}, x_{n}^{a p} d x\right) \rightarrow L_{p}\left(\mathbb{R}_{+}^{n}, x_{n}^{a p} d x\right) \text { boundedly } \\
& \text { for each } p \in(1, \infty) \text { and each } a \in(-1 / p, 1-1 / p) \text {. }
\end{aligned}
$$

The corresponding estimate for the norm $S$ results as well. By duality, it follows that $S^{*}$ enjoys the same property and, hence, the operator $T$ is bounded on $L_{p}\left(\mathbb{R}_{+}^{n}, x_{n}^{a p} d x\right)$ for each $p \in(1, \infty)$ and $a \in(-1 / p, 1-1 / p)$, thanks to (3.24) and Corollary 3.3. The fact that the operator norm of $T$ admits the desired estimate is implicit in the above reasoning and this finishes the proof of the proposition.

\subsection{Singular integral operators}

We need the analogue of Proposition 3.5 for the class of Mikhlin-Calderón-Zygmund singular integral operators. Recall that

$$
\mathcal{S} f(x)=p . v \cdot \int_{\mathbb{R}^{n}} k(x, x-y) f(y) d y, \quad x \in \mathbb{R}^{n},
$$

(where p.v. indicates that the integral is taken in the principal value sense, which means excluding balls centered at the singularity and then passing to the limit as the radii shrink to zero), is called a Mikhlin-Calderón-Zygmund operator (with a variable coefficient kernel) provided the function $k: \mathbb{R}^{n} \times\left(\mathbb{R}^{n} \backslash\{0\}\right) \rightarrow \mathbb{R}$ satisfies: 
(i) $k(x, \cdot) \in C^{\infty}\left(\mathbb{R}^{n} \backslash\{0\}\right)$ and, for almost each $x \in \mathbb{R}^{n}$,

$$
\max _{|\alpha| \leq 2 n}\left\|D_{z}^{\alpha} k(x, z)\right\|_{L_{\infty}\left(\mathbb{R}^{n} \times S^{n-1}\right)}<\infty
$$

where $S^{n-1}$ is the unit sphere in $\mathbb{R}^{n}$;

(ii) $k(x, \lambda z)=\lambda^{-n} k(x, z)$ for each $z \in \mathbb{R}^{n}$ and each $\lambda \in \mathbb{R}, \lambda>0$;

(iii) $\int_{S^{n-1}} k(x, \omega) d \omega=0$, where $d \omega$ indicates integration with respect to $\omega \in S^{n-1}$.

It is well-known that the Mikhlin-Calderón-Zygmund operator $\mathcal{S}$ and its commutator $[\mathcal{S}, b]$ with the operator of multiplication by a function $b \in \mathrm{BMO}\left(\mathbb{R}_{+}^{n}\right)$ are bounded operators in $L_{p}\left(\mathbb{R}_{+}^{n}\right)$ for each $1<p<\infty$. The norms of these operators admit the estimates

$$
\|\mathcal{S}\|_{\mathfrak{L}\left(L_{p}\left(\mathbb{R}_{+}^{n}\right)\right)} \leq c(n) p p^{\prime}, \quad\|[\mathcal{S}, b]\|_{\mathfrak{L}\left(L_{p}\left(\mathbb{R}_{+}^{n}\right)\right)} \leq c(n) p p^{\prime}[b]_{\mathrm{BMO}\left(\mathbb{R}_{+}^{n}\right)},
$$

where $c(n)$ depends only on $n$ and the quantity in (3.31). The first estimate in (3.32) goes back to the work of A. Calderón and A. Zygmund (cf., e.g., [8], [9]; see also the comment on p. 22 of [47] regarding the dependence on the parameter $p$ of the constants involved). The second estimate in (3.32) was originally proved for convolution type operators by R. Coifman, R. Rochberg and G. Weiss in [12] and a standard expansion in spherical harmonics allows to extend this result to the case of operators with variable-kernels of the type considered above.

We are interested in extending (3.32) to the weighted case, i.e. when the measure $d x$ on $\mathbb{R}_{+}^{n}$ is replaced by $x_{n}^{a p} d x$, where $1<p<\infty$ and $a \in(-1 / p, 1-1 / p)$. Parenthetically, we wish to point out that $a \in(-1 / p, 1-1 / p)$ corresponds precisely to the range of $a$ 's for which $w(x):=x_{n}^{a p}$ is a weight in Muckenhoupt's $A_{p}$ class, and that while in principle this observation can help with the goal just stated, we prefer to give a direct, self-contained proof.

Proposition 3.6 Retain the above conventions and hypotheses. Then the operator $\mathcal{S}$ and its commutator $[\mathcal{S}, b]$ with a function $b \in \mathrm{BMO}\left(\mathbb{R}_{+}^{n}\right)$ are bounded when acting from $L_{p}\left(\mathbb{R}_{+}^{n}, x_{n}^{a p} d x\right)$ into itself for each $p \in(1, \infty)$ and $a \in(-1 / p, 1-1 / p)$. The norms of these operators satisfy

$$
\begin{aligned}
& \|\mathcal{S}\|_{\mathfrak{L}\left(L_{p}\left(\mathbb{R}_{+}^{n}, x_{n}^{a p} d x\right)\right)} \leq c(n)\left(p p^{\prime}+\frac{1}{s(1-s)}\right) \\
& \|[\mathcal{S}, b]\|_{\mathfrak{L}\left(L_{p}\left(\mathbb{R}_{+}^{n}, x_{n}^{a p} d x\right)\right)} \leq c(n)\left(p p^{\prime}+\frac{1}{s(1-s)}\right)[b]_{\mathrm{BMO}\left(\mathbb{R}_{+}^{n}\right)} .
\end{aligned}
$$

Proof. Let $\chi_{j}$ be the characteristic function of the layer $2^{j / 2}<x_{n} \leq 2^{1+j / 2}, j=0, \pm 1, \ldots$, so that $\sum_{j \in \mathbb{Z}} \chi_{j}=2$. We then write $\mathcal{S}$ as the sum $\mathcal{S}_{1}+\mathcal{S}_{2}$, where

$$
\mathcal{S}_{1}:=\frac{1}{4} \sum_{|j-k| \leq 3} \chi_{j} \mathcal{S} \chi_{k} .
$$

The following chain of inequalities is evident 


$$
\begin{aligned}
\left\|\mathcal{S}_{1} f\right\|_{L_{p}\left(\mathbb{R}_{+}^{n}, x_{n}^{a p} d x\right)} & \leq\left(\sum_{j} \int_{\mathbb{R}_{+}^{n}} \chi_{j}(x)\left|\mathcal{S}\left(\sum_{|k-j| \leq 3} \chi_{k} f\right)(x)\right|^{p} x_{n}^{a p} d x\right)^{1 / p} \\
& \leq c(n)\left(\sum_{j} \int_{\mathbb{R}_{+}^{n}}\left|\mathcal{S}\left(\sum_{|k-j| \leq 3} \chi_{k} 2^{j a / 2} f\right)(x)\right|^{p} d x\right)^{1 / p} .
\end{aligned}
$$

In concert with the first estimate in (3.32), this entails

$$
\begin{aligned}
\left\|\mathcal{S}_{1} f\right\|_{L_{p}\left(\mathbb{R}_{+}^{n}, x_{n}^{a p} d x\right)} & \leq c(n) p p^{\prime}\left(\sum_{j} \int_{\mathbb{R}_{+}^{n}}\left(\sum_{|k-j| \leq 3} \chi_{k} 2^{j a / 2}|f|\right)^{p} d x\right)^{1 / p} \\
& \leq c(n) p p^{\prime}\left(\int_{\mathbb{R}_{+}^{n}}|f(x)|^{p} x_{n}^{a p} d x\right)^{1 / p},
\end{aligned}
$$

which is further equivalent to

$$
\left\|\mathcal{S}_{1}\right\|_{\mathfrak{L}\left(L_{p}\left(\mathbb{R}_{+}^{n}, x_{n}^{a p} d x\right)\right)} \leq c(n) p p^{\prime} .
$$

Applying the same argument to $[\mathcal{S}, b]$ and referring to (3.32), we arrive at

$$
\left\|\left[\mathcal{S}_{1}, b\right]\right\|_{\mathfrak{L}\left(L_{p}\left(\mathbb{R}_{+}^{n}, x_{n}^{a p} d x\right)\right)} \leq c(n) p p^{\prime}[b]_{\mathrm{BMO}\left(\mathbb{R}_{+}^{n}\right)} .
$$

It remains to obtain the analogues of (3.38) and (3.39) with $\mathcal{S}_{2}$ in place of $\mathcal{S}_{1}$. One can check directly that the modulus of the kernel of $\mathcal{S}_{2}$ does not exceed $c(n)|x-\bar{y}|^{-n}$ and that the modulus of the kernel of $\left[\mathcal{S}_{2}, b\right]$ is majorized by $c(n)|b(x)-b(y)||x-\bar{y}|^{-n}$. Then the desired conclusions follow from Corollary 3.3 and Proposition 3.5 .

\subsection{The Gagliardo extension operator}

Here we shall revisit a certain operator $T$, extending functions defined on $\mathbb{R}^{n-1}$ into functions defined on $\mathbb{R}_{+}^{n}$, first introduced by Gagliardo in [18]. Fix a smooth, radial, decreasing, even, non-negative function $\zeta$ in $\mathbb{R}^{n-1}$ such that $\zeta(t)=0$ for $|t| \geq 1$ and

$$
\int_{\mathbb{R}^{n-1}} \zeta(t) d t=1
$$

(A standard choice is $\zeta(t):=c \exp \left(-1 /\left(1-|t|^{2}\right)_{+}\right)$for a suitable $c$.) Following [18] we then define

$$
(T \varphi)\left(x^{\prime}, x_{n}\right)=\int_{\mathbb{R}^{n-1}} \zeta(t) \varphi\left(x^{\prime}+x_{n} t\right) d t, \quad\left(x^{\prime}, x_{n}\right) \in \mathbb{R}_{+}^{n},
$$

acting on functions $\varphi$ from $L_{1}\left(\mathbb{R}^{n-1}, l o c\right)$. To get started, we note that

$$
\begin{aligned}
\nabla_{x^{\prime}}(T \varphi)\left(x^{\prime}, x_{n}\right) & =\int_{\mathbb{R}^{n-1}} \zeta(t) \nabla \varphi\left(x^{\prime}+t x_{n}\right) d t \\
\frac{\partial}{\partial x_{n}}(T \varphi)\left(x^{\prime}, x_{n}\right) & =\int_{\mathbb{R}^{n-1}} \zeta(t) t \nabla \varphi\left(x^{\prime}+t x_{n}\right) d t
\end{aligned}
$$


and, hence, we have the estimate

$$
\left\|\nabla_{x}(T \varphi)\right\|_{L_{\infty}\left(\mathbb{R}_{+}^{n}\right)} \leq c\left\|\nabla_{x^{\prime}} \varphi\right\|_{L_{\infty}\left(\mathbb{R}^{n-1}\right)} .
$$

Refinements of (3.44) are contained in the Lemmas 3.7.3.8 below.

Lemma 3.7 (i) For all $x \in \mathbb{R}_{+}^{n}$ and for all multi-indices $\alpha$ with $|\alpha|>1$,

$$
\left|D_{x}^{\alpha}(T \varphi)(x)\right| \leq c x_{n}^{1-|\alpha|}[\nabla \varphi]_{\mathrm{BMO}\left(\mathbb{R}^{\mathrm{n}-1}\right)} .
$$

(ii) For all $x=\left(x^{\prime}, x_{n}\right) \in \mathbb{R}_{+}^{n}$,

$$
\left|(T \varphi)(x)-\varphi\left(x^{\prime}\right)\right| \leq c x_{n}[\nabla \varphi]_{\mathrm{BMO}\left(\mathbb{R}^{\mathrm{n}-1}\right)} .
$$

Proof. Rewriting (3.43) as

$$
\frac{\partial}{\partial x_{n}}(T \varphi)\left(x^{\prime}, x_{n}\right)=x_{n}^{1-n} \int_{\mathbb{R}^{n-1}} \zeta\left(\frac{\xi-x^{\prime}}{x_{n}}\right) \frac{\xi-x^{\prime}}{x_{n}}\left(\nabla \varphi(\xi)-f_{\left|z-x^{\prime}\right|<x_{n}} \nabla \varphi(z) d z\right) d \xi
$$

we obtain

$$
\left|D_{x}^{\gamma} \frac{\partial}{\partial x_{n}}(T \varphi)(x)\right| \leq c x_{n}^{-|\gamma|}[\nabla \varphi]_{\mathrm{BMO}\left(\mathbb{R}^{\mathrm{n}-1}\right)}
$$

for every non-zero multi-index $\gamma$. Furthermore, for $i=1, \ldots n-1$, by (3.42)

$$
\frac{\partial}{\partial x_{i}} \nabla_{x^{\prime}}(T \varphi)(x)=x_{n}^{1-n} \int_{\mathbb{R}^{n-1}} \partial_{i} \zeta\left(\frac{\xi-x^{\prime}}{x_{n}}\right)\left(\nabla \varphi(\xi)-f_{\left|z-x^{\prime}\right|<x_{n}} \nabla \varphi(z) d z\right) d \xi,
$$

where $\partial_{i}$ is the differentiation with respect to the $i$-th component of the argument. Hence once again

$$
\left|D_{x}^{\gamma} \frac{\partial}{\partial x_{i}} \nabla_{x^{\prime}}(T \varphi)(x)\right| \leq c x_{n}^{-|\gamma|-1}[\nabla \varphi]_{\mathrm{BMO}\left(\mathbb{R}^{\mathrm{n}-1}\right)},
$$

and the estimate claimed in (i) follows.

Finally, (ii) is a simple consequence of (i) and the fact that $\left.(T \varphi)\right|_{\mathbb{R}^{n-1}}=\varphi$.

Remark. In concert with Theorem 2 on p.62-63 in [47], formula (3.42) yields the pointwise estimate

$$
|\nabla(T \varphi)(x)| \leq c \mathcal{M}(\nabla \varphi)\left(x^{\prime}\right), \quad x=\left(x^{\prime}, x_{n}\right) \in \mathbb{R}_{+}^{n},
$$

where $\mathcal{M}$ is the classical Hardy-Littlewood maximal function (cf., e.g., Chapter I in [47]). As for higher order derivatives, an inspection of the above proof reveals that

$$
\left|D_{x}^{\alpha}(T \varphi)(x)\right| \leq c x_{n}^{1-|\alpha|}(\nabla \varphi)^{\#}\left(x^{\prime}\right), \quad\left(x^{\prime}, x_{n}\right) \in \mathbb{R}^{n},
$$

holds for each multi-index $\alpha$ with $|\alpha|>1$, where $(\cdot)^{\#}$ is the Fefferman-Stein sharp maximal function (cf. [17]). 
Lemma 3.8 If $\nabla_{x^{\prime}} \varphi \in \operatorname{BMO}\left(\mathbb{R}^{n-1}\right)$ then $\nabla(T \varphi) \in \operatorname{BMO}\left(\mathbb{R}_{+}^{n}\right)$ and

$$
[\nabla(T \varphi)]_{\mathrm{BMO}\left(\mathbb{R}_{+}^{n}\right)} \leq c\left[\nabla_{x^{\prime}} \varphi\right]_{\mathrm{BMO}\left(\mathbb{R}^{n-1}\right)}
$$

Proof. Since $(T \varphi)\left(x^{\prime}, x_{n}\right)$ is even with respect to $x_{n}$, it suffices to estimate $\left[\nabla_{x}(T \varphi)\right]_{\mathrm{BMO}\left(\mathbb{R}^{n}\right)}$. Let $Q_{r}$ denote a cube with side-length $r$ centered at the point $\eta=\left(\eta^{\prime}, \eta_{n}\right) \in \mathbb{R}^{n-1} \times \mathbb{R}$. Also let $Q_{r}^{\prime}$ be the projection of $Q_{r}$ on $\mathbb{R}^{n-1}$. Clearly,

$$
\nabla_{x^{\prime}}(T \varphi)\left(x^{\prime}, x_{n}\right)-\nabla_{x^{\prime}} \varphi\left(x^{\prime}\right)=x_{n}^{1-n} \int_{\mathbb{R}^{n-1}} \zeta\left(\frac{\xi-x^{\prime}}{x_{n}}\right)\left(\nabla \varphi(\xi)-\nabla \varphi\left(x^{\prime}\right)\right) d \xi
$$

Suppose that $\left|\eta_{n}\right|<2 r$ and write

$$
\begin{aligned}
\int_{Q_{r}}\left|\nabla_{x^{\prime}}(T \varphi)\left(x^{\prime}, x_{n}\right)-\nabla_{x^{\prime}} \varphi\left(x^{\prime}\right)\right| d x & \leq c r^{2-n} \int_{Q_{4 r}^{\prime}} \int_{Q_{4 r}^{\prime}}|\nabla \varphi(\xi)-\nabla \varphi(z)| d z d \xi \\
& \leq c r^{n}[\nabla \varphi]_{\mathrm{BMO}\left(\mathbb{R}^{n-1}\right)}
\end{aligned}
$$

Therefore, for $\left|\eta_{n}\right|<2 r$

$$
\begin{aligned}
f_{Q_{r}} f_{Q_{r}}\left|\nabla_{x^{\prime}} T \varphi(x)-\nabla_{y^{\prime}} T \varphi(y)\right| d x d y \leq & 2 f_{Q_{r}}\left|\nabla_{x^{\prime}} T \varphi(x)-\nabla \varphi\left(x^{\prime}\right)\right| d x \\
& +f_{Q_{r}^{\prime}} f_{Q_{r}^{\prime}}\left|\nabla \varphi\left(x^{\prime}\right)-\nabla \varphi\left(y^{\prime}\right)\right| d x^{\prime} d y^{\prime} \\
\leq & c[\nabla \varphi]_{\mathrm{BMO}\left(\mathbb{R}^{n-1}\right) .}
\end{aligned}
$$

Next, consider the case when $\left|\eta_{n}\right| \geq 2 r$ and let $x$ and $y$ be arbitrary points in $Q_{r}(\eta)$. Then, using the generic abbreviation $\bar{f}_{E}:=f_{E} f$, we may write

$$
\begin{aligned}
\left|\nabla_{x^{\prime}} T \varphi(x)-\nabla_{y^{\prime}} T \varphi(y)\right| & \leq \int_{\mathbb{R}^{n-1}}\left|x_{n}^{1-n} \zeta\left(\frac{\xi-x^{\prime}}{x_{n}}\right)-y_{n}^{1-n} \zeta\left(\frac{\xi-y^{\prime}}{y_{n}}\right)\right|\left|\nabla \varphi(\xi)-\bar{\nabla}_{Q_{2\left|\eta_{n}\right|}^{\prime}}\right| d \xi \\
& \leq \frac{c r}{\left|\eta_{n}\right|^{n}} \int_{Q_{2\left|\eta_{n}\right|}^{\prime}}\left|\nabla \varphi(\xi)-\bar{\nabla} \varphi_{Q_{2\left|\eta_{n}\right|}^{\prime}}\right| d \xi \\
& \leq c[\nabla \varphi]_{\mathrm{BMO}\left(\mathbb{R}^{n-1}\right)}
\end{aligned}
$$

Consequently, for $\left|\eta_{n}\right| \geq 2 r$,

$$
f_{Q_{r}} f_{Q_{r}}\left|\nabla_{x^{\prime}} T \varphi(x)-\nabla_{y^{\prime}} T \varphi(y)\right| d x d y \leq c[\nabla \varphi]_{\mathrm{BMO}\left(\mathbb{R}^{n-1}\right)}
$$

which, together with (3.56), gives

$$
\left[\nabla_{x^{\prime}} T \varphi\right]_{\mathrm{BMO}\left(\mathbb{R}^{n}\right)} \leq c[\nabla \varphi]_{\mathrm{BMO}\left(\mathbb{R}^{\mathrm{n}-1}\right)} .
$$

This inequality and (3.48), where $|\gamma|=0$, imply (3.53). 


\section{The Dirichlet problem in $\mathbb{R}_{+}^{n}$ for variable coefficient systems}

\subsection{Preliminaries}

For

$$
1<p<\infty, \quad-\frac{1}{p}<a<1-\frac{1}{p} \quad \text { and } \quad m \in \mathbb{N}
$$

we let $V_{p}^{m, a}\left(\mathbb{R}_{+}^{n}\right)$ denote the weighted Sobolev space associated with the norm

$$
\|u\|_{V_{p}^{m, a}\left(\mathbb{R}_{+}^{n}\right)}:=\left(\sum_{0 \leq|\beta| \leq m} \int_{\mathbb{R}_{+}^{n}}\left|x_{n}^{|\beta|-m} D^{\beta} u(x)\right|^{p} x_{n}^{p a} d x\right)^{1 / p} .
$$

It is easily proved that $C_{0}^{\infty}\left(\mathbb{R}_{+}^{n}\right)$ is dense in $V_{p}^{m, a}\left(\mathbb{R}_{+}^{n}\right)$. Moreover, by the one-dimensional Hardy's inequality (see, for instance, [31], formula $(1.3 / 1)$ ), we have

$$
\|u\|_{V_{p}^{m, a}\left(\mathbb{R}_{+}^{n}\right)} \leq c s^{-1}\left(\sum_{|\beta|=m} \int_{\mathbb{R}_{+}^{n}}\left|D^{\beta} u(x)\right|^{p} x_{n}^{p a} d x\right)^{1 / p} \quad \text { for } u \in C_{0}^{\infty}\left(\mathbb{R}_{+}^{n}\right) .
$$

The dual of $V_{p^{\prime}}^{m,-a}\left(\mathbb{R}_{+}^{n}\right)$ will be denoted by $V_{p}^{m, a}\left(\mathbb{R}_{+}^{n}\right)$, where $1 / p+1 / p^{\prime}=1$.

Consider now the operator

$$
L\left(x, D_{x}\right) u:=\sum_{0 \leq|\alpha|,|\beta| \leq m} D_{x}^{\alpha}\left(A_{\alpha \beta}(x) x_{n}^{|\alpha|+|\beta|-2 m} D_{x}^{\beta} u\right)
$$

where $A_{\alpha \beta}$ are $\mathbb{C}^{l \times l}$-valued functions in $L_{\infty}\left(\mathbb{R}_{+}^{n}\right)$. We shall use the notation $\stackrel{\circ}{L}\left(x, D_{x}\right)$ for the principal part of $L\left(x, D_{x}\right)$, i.e.

$$
\stackrel{\circ}{L}\left(x, D_{x}\right) u:=\sum_{|\alpha|=|\beta|=m} D_{x}^{\alpha}\left(A_{\alpha \beta}(x) D_{x}^{\beta} u\right) .
$$

\subsection{Solvability and regularity result}

Lemma 4.1 Assume that there exists $\kappa=$ const $>0$ such that the coercivity condition

$$
\Re \int_{\mathbb{R}_{+}^{n}} \sum_{|\alpha|=|\beta|=m}\left\langle A_{\alpha \beta}(x) D^{\beta} u(x), D^{\alpha} u(x)\right\rangle_{\mathbb{C}^{l}} d x \geq \kappa \sum_{|\gamma|=m}\left\|D^{\gamma} u\right\|_{L_{2}\left(\mathbb{R}_{+}^{n}\right)}^{2},
$$

holds for all $u \in C_{0}^{\infty}\left(\mathbb{R}_{+}^{n}\right)$, and that

$$
\sum_{|\alpha|=|\beta|=m}\left\|A_{\alpha \beta}\right\|_{L_{\infty}\left(\mathbb{R}_{+}^{n}\right)} \leq \kappa^{-1}
$$

(i) Let $p \in(1, \infty)$ and $-1 / p<a<1-1 / p$ and suppose that

$$
\frac{1}{s(1-s)} \sum_{\substack{|\alpha|+|\beta|<2 m \\ 0 \leq|\alpha|,|\beta| \leq m}}\left\|A_{\alpha \beta}\right\|_{L_{\infty}\left(\mathbb{R}_{+}^{n}\right)}+\sum_{|\alpha|=|\beta|=m}\left[A_{\alpha \beta}\right]_{\mathrm{BMO}\left(\mathbb{R}_{+}^{n}\right)} \leq \delta,
$$

where $\delta$ satisfies 


$$
\left(p p^{\prime}+\frac{1}{s(1-s)}\right) \delta<c(n, m, \kappa)
$$

with a sufficiently small constant $c(n, m, \kappa)$ and $s=1-a-1 / p$.

Then the operator

$$
L=L\left(x, D_{x}\right): V_{p}^{m, a}\left(\mathbb{R}_{+}^{n}\right) \longrightarrow V_{p}^{-m, a}\left(\mathbb{R}_{+}^{n}\right)
$$

is an isomorphism.

(ii) Let $p_{i} \in(1, \infty)$ and $-1 / p_{i}<a_{i}<1-1 / p_{i}$, where $i=1,2$. Suppose that 4.9 ) holds with $p_{i}$ and $s_{i}=1-a_{i}-1 / p_{i}$ in place of $p$ and $s$. If $u \in V_{p_{1}}^{m, a_{1}}\left(\mathbb{R}_{+}^{n}\right)$ is such that $L u \in V_{p_{1}}^{-m, a_{1}}\left(\mathbb{R}_{+}^{n}\right) \cap$ $V_{p_{2}}^{-m, a_{2}}\left(\mathbb{R}_{+}^{n}\right)$, then $u \in V_{p_{2}}^{m, a_{2}}\left(\mathbb{R}_{+}^{n}\right)$.

Proof. The fact that the operator (4.10) is continuous is obvious. Also, the existence of a bounded inverse $L^{-1}$ for $p=2$ and $a=0$ follows from (4.6) and (4.8)-(4.9) with $p=2, a=0$, which allow us to implement the Lax-Milgram lemma.

We shall use the notation $\stackrel{\circ}{L}_{y}$ for the operator $\stackrel{\circ}{L}\left(y, D_{x}\right)$, corresponding to (4.5) in which the coefficients have been frozen at $y \in \mathbb{R}_{+}^{n}$, and the notation $G_{y}$ for the solution operator for the Dirichlet problem for $\stackrel{\circ}{L}_{y}$ in $\mathbb{R}_{+}^{n}$ with homogeneous boundary conditions. Next, given $u \in V_{p}^{m, a}\left(\mathbb{R}_{+}^{n}\right)$, set $f:=L u \in V_{p}^{-m, a}\left(\mathbb{R}_{+}^{n}\right)$ so that

$$
\begin{cases}L(x, D) u=f & \text { in } \mathbb{R}_{+}^{n}, \\ \frac{\partial^{j} u}{\partial x_{n}^{j}}\left(x^{\prime}, 0\right)=0 & \text { on } \mathbb{R}^{n-1}, 0 \leq j \leq m-1 .\end{cases}
$$

Applying the trick used for the first time in [10], we may write

$$
u(x)=\left(G_{y} f\right)(x)-\left(G_{y}\left(\stackrel{\circ}{L}-\stackrel{\circ}{L}_{y}\right) u\right)(x)-\left(G_{y}(L-\stackrel{\circ}{L}) u\right)(x), \quad x \in \mathbb{R}_{+}^{n} .
$$

We desire to use (4.12) in order to express $u$ in terms of $f$ (cf. (4.27)-(4.28) below) via integral operators whose norms we can control.

First, we claim that whenever $|\gamma|=m$, the norm of the operator

$$
\left.V_{p}^{m, a}\left(\mathbb{R}_{+}^{n}\right) \ni u \mapsto D_{x}^{\gamma}\left(G_{y}\left(\stackrel{\circ}{L}-\stackrel{\circ}{L}_{y}\right) u\right)(x)\right|_{x=y} \in L_{p}\left(\mathbb{R}_{+}^{n}, y_{n}^{a p} d y\right)
$$

does not exceed

$$
C\left(p p^{\prime}+\frac{1}{s(1-s)}\right) \sum_{|\alpha|=|\beta|=m}\left[A_{\alpha \beta}\right]_{\mathrm{BMO}\left(\mathbb{R}_{+}^{n}\right)} .
$$

Given the hypotheses under which we operate, the expression (4.14) is therefore small if $\delta$ is small.

In what follows, we denote by $G_{y}(x, z)$ the integral kernel of $G_{y}$ and integrate by parts in order to move derivatives of the form $D_{z}^{\alpha}$ with $|\alpha|=m$ from $\left(\stackrel{\circ}{L}-\stackrel{\circ}{L}_{y}\right) u$ onto $G_{y}(x, z)$ (the absence of boundary terms is due to the fact that $G_{y}(x, \cdot)$ satisfies homogeneous Dirichlet boundary conditions). That (4.14) bounds the norm of (4.13) can now be seen by combining Theorem 2.1 with (3.21) and Proposition 3.6.

Let $\gamma$ and $\alpha$ be multi-indices with $|\gamma|=m,|\alpha| \leq m$ and consider the assignment 


$$
\left.C_{0}^{\infty}\left(\mathbb{R}_{+}^{n}\right) \ni \Psi \mapsto\left(D_{x}^{\gamma} \int_{\mathbb{R}_{+}^{n}} G_{y}(x, z) D_{z}^{\alpha} \frac{\Psi(z)}{z_{n}^{m-|\alpha|}} d z\right)\right|_{x=y} .
$$

After integrating by parts, the action of this operator can be rewritten in the form

$$
\left.\left(D_{x}^{\gamma} \int_{\mathbb{R}_{+}^{n}}\left[\left(\frac{-1}{i} \frac{\partial}{\partial z_{n}}\right)^{m-|\alpha|}\left(-D_{z}\right)^{\alpha} G_{y}(x, z)\right] \Gamma_{\alpha}(z) d z\right)\right|_{x=y}
$$

where

$$
\Gamma_{\alpha}(z):=\left\{\begin{array}{l}
\Psi(z), \quad \text { if } \quad|\alpha|=m, \\
\frac{(-1)^{m-|\alpha|}}{(m-|\alpha|-1) !} \int_{z_{n}}^{\infty}\left(t-z_{n}\right)^{m-|\alpha|-1} \frac{\Psi\left(z^{\prime}, t\right)}{t^{m-|\alpha|}} d t, \quad \text { if } \quad|\alpha|<m .
\end{array}\right.
$$

Using Theorem 2.1 along with (3.21) and Proposition 3.6. we may therefore conclude that

$$
\begin{array}{r}
\left\|\left.\left(D_{x}^{\gamma} \int_{\mathbb{R}_{+}^{n}}\left[\left(\frac{-1}{i} \frac{\partial}{\partial z_{n}}\right)^{m-|\alpha|}\left(-D_{z}\right)^{\alpha} G_{y}(x, z)\right] \Gamma_{\alpha}(z) d z\right)\right|_{x=y}\right\|_{L_{p}\left(\mathbb{R}_{+}^{n}, y_{n}^{a p} d y\right)} \\
\leq C\left(p p^{\prime}+\frac{1}{s(1-s)}\right)\left\|\Gamma_{\alpha}\right\|_{L_{p}\left(\mathbb{R}_{+}^{n}, x_{n}^{a p} d x\right)} .
\end{array}
$$

On the other hand, Hardy's inequality gives

$$
\left\|\Gamma_{\alpha}\right\|_{L_{p}\left(\mathbb{R}_{+}^{n}, x_{n}^{a p} d x\right)} \leq \frac{C}{1-s}\|\Psi\|_{L_{p}\left(\mathbb{R}_{+}^{n}, x_{n}^{a p} d x\right)}
$$

and, hence, the operator (4.15) can be extended from $C_{0}^{\infty}\left(\mathbb{R}_{+}^{n}\right)$ as a bounded operator in $L_{p}\left(\mathbb{R}_{+}^{n}, x_{n}^{a p} d x\right)$ and its norm is majorized by

$$
\frac{C}{1-s}\left(p p^{\prime}+\frac{1}{s(1-s)}\right)
$$

Next, given an arbitrary $u \in V_{p}^{m, a}\left(\mathbb{R}_{+}^{n}\right)$, we let $\Psi=\Psi_{\alpha \beta}$ in (4.15) with

$$
\Psi_{\alpha \beta}(z):=z_{n}^{|\beta|-m} A_{\alpha \beta} D^{\beta} u(z), \quad|\alpha|+|\beta|<2 m,
$$

and conclude that the norm of the operator

$$
\left.V_{p}^{m, a}\left(\mathbb{R}_{+}^{n}\right) \ni u \mapsto D_{x}^{\gamma}\left(G_{y}(L-\stackrel{\circ}{L}) u\right)(x)\right|_{x=y} \in L_{p}\left(\mathbb{R}_{+}^{n}, y_{n}^{a p} d y\right)
$$

does not exceed

$$
\frac{C}{1-s}\left(p p^{\prime}+\frac{1}{s(1-s)}\right) \sum_{\substack{|\alpha|+|\beta|<2 m \\ 0 \leq|\alpha|,|\beta| \leq m}}\left\|A_{\alpha \beta}\right\|_{L_{\infty}\left(\mathbb{R}_{+}^{n}\right)} .
$$

It is well-known (cf. $(1.1 .10 / 6)$ on p. 22 of [31]) that any $u \in V_{p}^{m, a}\left(\mathbb{R}_{+}^{n}\right)$ can be represented in the form

$$
u=K\left\{D^{\sigma} u\right\}_{|\sigma|=m}
$$


where $K$ is a linear operator with the property that

$$
D^{\alpha} K: L_{p}\left(\mathbb{R}_{+}^{n}, x_{n}^{a p} d x\right) \longrightarrow L_{p}\left(\mathbb{R}_{+}^{n}, x_{n}^{a p} d x\right)
$$

is bounded for every multi-index $\alpha$ with $|\alpha|=m$. In particular, by (4.3),

$$
\left\|K\left\{D^{\sigma} u\right\}_{|\sigma|=m}\right\|_{V_{p}^{m, a}\left(\mathbb{R}_{+}^{n}\right)} \leq C s^{-1}\left\|\left\{D^{\sigma} u\right\}_{|\sigma|=m}\right\|_{L_{p}\left(\mathbb{R}_{+}^{n}, x_{n}^{a p} d x\right)} .
$$

At this stage, we transform the identity (4.12) in the following fashion. First, we express the two $u$ 's occurring inside the Green operator $G_{y}$ in the left-hand side of (4.12) as in (4.24). Second, for each multi-index $\gamma$ with $|\gamma|=m$, we apply $D^{\gamma}$ to both sides of (4.12) and, finally, set $x=y$. The resulting identity reads

$$
\left\{D^{\gamma} u\right\}_{|\gamma|=m}+S\left\{D^{\sigma} u\right\}_{|\sigma|=m}=Q f
$$

where $Q$ is a bounded operator from $V_{p}^{-m, a}\left(\mathbb{R}_{+}^{n}\right)$ into $L_{p}\left(\mathbb{R}_{+}^{n}, x_{n}^{a p} d x\right)$ and $S$ is a linear operator mapping $L_{p}\left(\mathbb{R}_{+}^{n}, x_{n}^{a p} d x\right)$ into itself. Furthermore, on account of (4.13)-(4.14), (4.22)-(4.23) and (4.26), we can bound $\|S\|_{\mathfrak{L}\left(L_{p}\left(\mathbb{R}_{+}^{n}, x_{n}^{a p} d x\right)\right)}$ by

$$
C\left(p p^{\prime}+\frac{1}{s(1-s)}\right)\left(\sum_{|\alpha|=|\beta|=m}\left[A_{\alpha \beta}\right]_{\mathrm{BMO}\left(\mathbb{R}_{+}^{n}\right)}+\frac{1}{s(1-s)} \sum_{\substack{|\alpha|+|\beta|<2 m \\ 0 \leq|\alpha|,|\beta| \leq m}}\left\|A_{\alpha \beta}\right\|_{L^{\infty}\left(\mathbb{R}_{+}^{n}\right)}\right) .
$$

Owing to (4.8)-(4.9) and with the integral representation formula (4.27) and the bound (4.28) in hand, a Neumann series argument and standard functional analysis allow us to simultaneously settle the claims (i) and (ii) in the statement of the lemma.

\section{The Dirichlet problem in a special Lipschitz domain}

In this section as well as in subsequent ones, we shall work with an unbounded domain of the form

$$
G=\left\{X=\left(X^{\prime}, X_{n}\right) \in \mathbb{R}^{n}: X^{\prime} \in \mathbb{R}^{n-1}, X_{n}>\varphi\left(X^{\prime}\right)\right\},
$$

where $\varphi: \mathbb{R}^{n-1} \rightarrow \mathbb{R}$ is a Lipschitz function.

\subsection{The space $\operatorname{BMO}(G)$}

The space of functions of bounded mean oscillations in $G$ can be introduced in a similar fashion to the case $G=\mathbb{R}_{+}^{n}$. Specifically, a locally integrable function on $G$ belongs to the space $\operatorname{BMO}(G)$ if

$$
[f]_{\mathrm{BMO}(G)}:=\sup _{\{B\}} f_{B \cap G}\left|f(X)-f_{B \cap G} f(Y) d Y\right| d X<\infty
$$

where the supremum is taken over all balls $B$ centered at points in $\bar{G}$. Much as before,

$$
[f]_{\mathrm{BMO}(G)} \sim \sup _{\{B\}} f_{B \cap G} f_{B \cap G}|f(X)-f(Y)| d X d Y .
$$

This implies the equivalence relation

$$
[f]_{\mathrm{BMO}(G)} \sim[f \circ \lambda]_{\mathrm{BMO}\left(\mathbb{R}_{+}^{n}\right)}
$$


for each bi-Lipschitz diffeomorphism $\lambda$ of $\mathbb{R}_{+}^{n}$ onto $G$. As direct consequences of definitions, we also have

$$
\begin{aligned}
{\left[\prod_{1 \leq j \leq N} f_{j}\right]_{\mathrm{BMO}(G)} } & \leq c\|f\|_{L_{\infty}(G)}^{N-1}[f]_{\mathrm{BMO}(G)}, \text { where } f=\left(f_{1}, \ldots, f_{N}\right) \\
{\left[f^{-1}\right]_{\mathrm{BMO}(G)} } & \leq c\left\|f^{-1}\right\|_{L_{\infty}(G)}^{2}[f]_{\mathrm{BMO}(G)} .
\end{aligned}
$$

\subsection{A bi-Lipschitz map $\lambda: \mathbb{R}_{+}^{n} \rightarrow G$ and its inverse}

Let $\varphi: \mathbb{R}^{n-1} \rightarrow \mathbb{R}$ be the Lipschitz function whose graph is $\partial G$ and set $M:=\|\nabla \varphi\|_{L_{\infty}\left(\mathbb{R}^{n-1}\right)}$. Next, let $T$ be the extension operator defined as in (3.41) and, for a fixed, sufficiently large constant $C>0$, consider the Lipschitz mapping

$$
\lambda: \mathbb{R}_{+}^{n} \ni\left(x^{\prime}, x_{n}\right) \mapsto\left(X^{\prime}, X_{n}\right) \in G
$$

defined by the equalities

$$
X^{\prime}:=x^{\prime}, \quad X_{n}:=C M x_{n}+(T \varphi)\left(x^{\prime}, x_{n}\right)
$$

(see [32], §6.5.1 and an earlier, less accessible, reference [33]). The Jacobi matrix of $\lambda$ is given by

$$
\lambda^{\prime}=\left(\begin{array}{cc}
I & 0 \\
\nabla_{x^{\prime}}(T \varphi) & C M+\partial(T \varphi) / \partial x_{n}
\end{array}\right)
$$

where $I$ is the identity $(n-1) \times(n-1)$-matrix. Since $\left|\partial(T \varphi) / \partial x_{n}\right| \leq c M$ by (3.43), it follows that $\operatorname{det} \lambda^{\prime}>(C-c) M>0$.

Next, thanks to (3.46) and (5.7)-(5.8) we have

$$
X_{n}-\varphi\left(X^{\prime}\right) \sim x_{n}
$$

Also, based on (3.53) we may write

$$
\left[\lambda^{\prime}\right]_{\mathrm{BMO}\left(\mathbb{R}_{+}^{n}\right)} \leq c[\nabla \varphi]_{\mathrm{BMO}\left(\mathbb{R}^{n-1}\right)}
$$

and further, by (3.44) and (3.45),

$$
\left\|D^{\alpha} \lambda^{\prime}(x)\right\|_{\mathbb{R}^{n \times n}} \leq c(M) x_{n}^{-|\alpha|}[\nabla \varphi]_{\mathrm{BMO}\left(\mathbb{R}^{n-1}\right)}, \quad \forall \alpha:|\alpha| \geq 1 .
$$

Next, by closely mimicking the proof of Proposition 2.6 from [34] it is possible to show the existence of the inverse Lipschitz mapping $\varkappa:=\lambda^{-1}: G \rightarrow \mathbb{R}_{+}^{n}$. Owing to (5.4), the inequality (5.11) implies

$$
\left[\lambda^{\prime} \circ \varkappa\right]_{\mathrm{BMO}(G)} \leq c[\nabla \varphi]_{\mathrm{BMO}\left(\mathbb{R}^{n-1}\right)} .
$$

Furthermore, (5.12) is equivalent to

$$
\left\|\left(D^{\alpha} \lambda^{\prime}\right)(\varkappa(X))\right\|_{\mathbb{R}^{n \times n}} \leq c(M, \alpha)\left(X_{n}-\varphi\left(X^{\prime}\right)\right)^{-|\alpha|}[\nabla \varphi]_{\mathrm{BMO}\left(\mathbb{R}^{n-1}\right)},
$$

whenever $|\alpha|>0$. Since $\varkappa^{\prime}=\left(\lambda^{\prime} \circ \varkappa\right)^{-1}$ we obtain from (5.6) and (5.13)

$$
\left[\varkappa^{\prime}\right]_{\mathrm{BMO}(G)} \leq c[\nabla \varphi]_{\mathrm{BMO}\left(\mathbb{R}^{n-1}\right)}
$$


On the other hand, using $\varkappa^{\prime}=\left(\lambda^{\prime} \circ \varkappa\right)^{-1}$ and (5.14) one can prove by induction on the order of differentiation that

$$
\left\|D^{\alpha} \varkappa^{\prime}(X)\right\|_{\mathbb{R}^{n \times n}} \leq c(M, \alpha)\left(X_{n}-\varphi\left(X^{\prime}\right)\right)^{-|\alpha|}[\nabla \varphi]_{\mathrm{BMO}\left(\mathbb{R}^{n-1}\right)}
$$

for all $X \in G$ if $|\alpha|>0$.

\subsection{The space $V_{p}^{m, a}(G)$}

Analogously to $V_{p}^{m, a}\left(\mathbb{R}_{+}^{n}\right)$, we define the weighted Sobolev space $V_{p}^{m, a}(G)$ naturally associated with the norm

$$
\|\mathcal{U}\|_{V_{p}^{m, a}(G)}:=\left(\sum_{0 \leq|\gamma| \leq m} \int_{G}\left|\left(X_{n}-\varphi\left(X^{\prime}\right)\right)^{|\gamma|-m} D^{\gamma} \mathcal{U}(X)\right|^{p}\left(X_{n}-\varphi\left(X^{\prime}\right)\right)^{p a} d X\right)^{1 / p}
$$

Replacing the function $X_{n}-\varphi\left(X^{\prime}\right)$ by either $\rho(X):=\operatorname{dist}(X, \partial G)$, or by the so-called regularized distance function $\rho_{\text {reg }}(X)$ (defined as on pp.170-171 of [47]), yields equivalent norms on $V_{p}^{m, a}(G)$. Based on a standard localization argument involving a cut-off function vanishing near $\partial G$ (for example, take $\eta\left(\rho_{\text {reg }} / \varepsilon\right)$ where $\eta \in C_{0}^{\infty}(\mathbb{R})$ satisfies $\eta(t)=0$ for $|t|<1$ and $\eta(t)=1$ for $|t|>2$ ) one can show that $C_{0}^{\infty}(G)$ is dense in $V_{p}^{m, a}(G)$.

Next, we observe that for each $\mathcal{U} \in C_{0}^{\infty}(G)$,

$$
C s\|\mathcal{U}\|_{V_{p}^{m, a}(G)} \leq\left(\sum_{|\gamma|=m} \int_{G}\left|D^{\gamma} \mathcal{U}(X)\right|^{p}\left(X_{n}-\varphi\left(X^{\prime}\right)\right)^{p a} d X\right)^{1 / p} \leq\|\mathcal{U}\|_{V_{p}^{m, a}(G)}
$$

where, as before, $s=1-a-1 / p$. Indeed, for each multi-index $\gamma$ with $0 \leq|\gamma| \leq m$, the onedimensional Hardy's inequality gives

$$
\begin{aligned}
\int_{G}\left|\left(X_{n}-\varphi\left(X^{\prime}\right)\right)^{|\gamma|-m} D^{\gamma} \mathcal{U}(X)\right|^{p}\left(X_{n}-\varphi\left(X^{\prime}\right)\right)^{p a} d X \\
\leq(C / s)^{p} \sum_{|\alpha|=m} \int_{G}\left|D^{\alpha} \mathcal{U}(X)\right|^{p}\left(X_{n}-\varphi\left(X^{\prime}\right)\right)^{p a} d X,
\end{aligned}
$$

and the first inequality in (5.18) follows readily from it. Also, the second inequality in (5.18) is a trivial consequence of (5.17).

Going further, we aim to establish that

$$
c_{1}\|u\|_{V_{p}^{m, a}\left(\mathbb{R}_{+}^{n}\right)} \leq\|u \circ \varkappa\|_{V_{p}^{m, a}(G)} \leq c_{2}\|u\|_{V_{p}^{m, a}\left(\mathbb{R}_{+}^{n}\right)},
$$

where $c_{1}$ and $c_{2}$ do not depend on $p$ and $s$, whereas $\varkappa: G \longrightarrow \mathbb{R}_{+}^{n}$ is the map introduced in $\S 5.2$. Clearly, it suffices to prove the upper estimate for $\|u \circ \varkappa\|_{V_{p}^{m, a}(G)}$ in (5.20). As a preliminary matter, we remark that

$$
\begin{aligned}
D^{\gamma}(u(\varkappa(X)))= & \left(\left(\varkappa^{\prime *}(X) \xi\right)_{\xi=D}^{\gamma} u\right)(\varkappa(X)) \\
& +\sum_{1 \leq|\tau|<|\gamma|}\left(D^{\tau} u\right)(\varkappa(X)) \sum_{\sigma} c_{\sigma} \prod_{i=1}^{n} \prod_{j} D^{\sigma_{i j}} \varkappa_{i}(X),
\end{aligned}
$$


where

$$
\sum_{i, j} \sigma_{i j}=\gamma, \quad\left|\sigma_{i j}\right| \geq 1, \quad \sum_{i, j}\left(\left|\sigma_{i j}\right|-1\right)=|\gamma|-|\tau| .
$$

In turn, (5.21)-(5.22) and (5.16) allow us to conclude that

$$
\left|D^{\gamma}(u(\varkappa(X)))\right| \leq c \sum_{1 \leq|\tau| \leq|\gamma|} x_{n}^{|\tau|-|\gamma|}\left|D^{\tau} u(x)\right|,
$$

which, in view of (5.10), yields the desired conclusion.

Finally, we set

$$
V_{p}^{-m, a}(G):=\left(V_{p^{\prime}}^{m,-a}(G)\right)^{*}
$$

where, as usual, $p^{\prime}=p /(p-1)$.

\subsection{Solvability and regularity result for the Dirichlet problem in the domain $G$}

Let us consider the differential operator

$$
\mathcal{L} \mathcal{U}=\mathcal{L}\left(X, D_{X}\right) \mathcal{U}=\sum_{|\alpha|=|\beta|=m} D^{\alpha}\left(\mathfrak{A}_{\alpha \beta}(X) D^{\beta} \mathcal{U}\right), \quad X \in G
$$

whose matrix-valued coefficients satisfy

$$
\sum_{|\alpha|=|\beta|=m}\left\|\mathfrak{A}_{\alpha \beta}\right\|_{L_{\infty}(G)} \leq \kappa^{-1}
$$

This operator generates the sesquilinear form $\mathcal{L}(\cdot, \cdot): V_{p}^{m, a}(G) \times V_{p^{\prime}}^{m,-a}(G) \rightarrow \mathbb{C}$ where $p^{\prime}$ is the conjugate exponent of $p$, defined by

$$
\mathcal{L}(\mathcal{U}, \mathcal{V}):=\sum_{|\alpha|=|\beta|=m} \int_{G}\left\langle\mathfrak{A}_{\alpha \beta}(X) D^{\beta} \mathcal{U}(X), D^{\alpha} \mathcal{V}(X)\right\rangle d X .
$$

We assume that the inequality

$$
\Re \mathcal{L}(\mathcal{U}, \mathcal{U}) \geq \kappa \sum_{|\gamma|=m}\left\|D^{\gamma} \mathcal{U}\right\|_{L_{2}(G)}^{2}
$$

holds for all $\mathcal{U} \in V_{2}^{m, 0}(G)$.

Lemma 5.1 (i) Let $p \in(1, \infty),-1 / p<a<1-1 / p$ and $s:=1-a-1 / p$. Suppose that

$$
[\nabla \varphi]_{\mathrm{BMO}\left(\mathbb{R}^{n-1}\right)}+\sum_{|\alpha|=|\beta|=m}\left[\mathfrak{A}_{\alpha \beta}\right]_{\mathrm{BMO}(G)} \leq \delta
$$

where $\delta$ satisfies

$$
\left(p p^{\prime}+\frac{1}{s(1-s)}\right) \frac{\delta}{s(1-s)}<C\left(n, m, \kappa,\|\nabla \varphi\|_{L_{\infty}\left(\mathbb{R}^{n-1}\right)}\right)
$$


with a sufficiently small constant $C$, independent of $p$ and $s$. In the case $m=1$ the factor $\delta / s(1-s)$ in (5.30) can be replaced by $\delta$.

Then the operator

$$
\mathcal{L}\left(X, D_{X}\right): V_{p}^{m, a}(G) \longrightarrow V_{p}^{-m, a}(G)
$$

is an isomorphism.

(ii) Let $p_{i} \in(1, \infty)$ and $-1 / p_{i}<a_{i}<1-1 / p_{i}$, where $i=1,2$. Suppose that (4.9) holds with $p_{i}$ and $s_{i}=1-a_{i}-1 / p_{i}$ in place of $p$ and $s$. If $\mathcal{U} \in V_{p_{1}}^{m, a_{1}}(G)$ and $\mathcal{L} \mathcal{U} \in V_{p_{1}}^{-m, a_{1}}(G) \cap V_{p_{2}}^{-m, a_{2}}(G)$, then $\mathcal{U} \in V_{p_{2}}^{m, a_{2}}(G)$.

Proof. We shall extensively use the flattening mapping $\lambda$ and its inverse studied in $§ 5.2$. The assertions (i) and (ii) will follow directly from Lemma 4.1 as soon as we show that the operator $L$ defined in $\mathbb{R}_{+}^{n}$ by

$$
L(\mathcal{U} \circ \lambda):=(\mathcal{L} \mathcal{U}) \circ \lambda
$$

satisfies all the hypotheses in that lemma. The sesquilinear form corresponding to the operator $L$ will be denoted by $L(u, v)$.

Set $u(x):=\mathcal{U}(\lambda(x)), v(x):=\mathcal{V}(\lambda(x))$ and note that the identity (5.21) implies

$$
\begin{aligned}
& D^{\beta} \mathcal{U}(X)=\left(\left(\varkappa^{\prime *}(\lambda(x)) \xi\right)_{\xi=D}^{\beta} u\right)(x)+\sum_{1 \leq|\tau|<|\beta|} K_{\beta \tau}(x) x_{n}^{|\tau|-|\beta|} D^{\tau} u(x), \\
& D^{\alpha} \mathcal{V}(X)=\left(\left(\varkappa^{* *}(\lambda(x)) \xi\right)_{\xi=D}^{\alpha} v\right)(x)+\sum_{1 \leq|\tau|<|\alpha|} K_{\alpha \tau}(x) x_{n}^{|\tau|-|\alpha|} D^{\tau} v(x),
\end{aligned}
$$

where, thanks to (5.16), the coefficients $K_{\gamma \tau}$ satisfy

$$
\left\|K_{\gamma \tau}\right\|_{L_{\infty}\left(\mathbb{R}_{+}^{n}\right)} \leq c[\nabla \varphi]_{\mathrm{BMO}\left(\mathbb{R}^{n-1}\right)} .
$$

Plugging (5.33) and (5.34) into the definition of $\mathcal{L}(\mathcal{U}, \mathcal{V})$, we arrive at

$$
\mathcal{L}(\mathcal{U}, \mathcal{V})=L_{0}(u, v)+\sum_{\substack{1 \leq|\alpha|,|\beta| \leq m \\|\alpha|+|\beta|<2 m}} \int_{\mathbb{R}_{+}^{n}}\left\langle A_{\alpha \beta}(x) x_{n}^{|\alpha|+|\beta|-2 m} D^{\beta} u(x), D^{\alpha} v(x)\right\rangle d x,
$$

where

$$
L_{0}(u, v)=\sum_{|\alpha|=|\beta|=m} \int_{\mathbb{R}_{+}^{n}}\left\langle\left(\mathfrak{A}_{\alpha \beta} \circ \lambda\right)\left(\left(\mathcal{\varkappa}^{*} \circ \lambda\right) \xi\right)_{\xi=D}^{\beta} u,\left(\left(\varkappa^{* *} \circ \lambda\right) \xi\right)_{\xi=D}^{\alpha} v\right\rangle \operatorname{det} \lambda^{\prime} d x .
$$

It follows from (5.33)-(5.35) that the coefficient matrices $A_{\alpha \beta}$ obey

$$
\sum_{\substack{1 \leq|\alpha|,|\beta| \leq m \\|\alpha|+|\beta|<2 m}}\left\|A_{\alpha \beta}\right\|_{L_{\infty}\left(\mathbb{R}_{+}^{n}\right)} \leq c \kappa^{-1}[\nabla \varphi]_{\mathrm{BMO}\left(\mathbb{R}^{n-1}\right)}
$$

where $c$ depends on $m, n$, and $\|\nabla \varphi\|_{L_{\infty}\left(\mathbb{R}^{n-1}\right)}$. We can write the form $L_{0}(u, v)$ as

$$
\sum_{|\alpha|=|\beta|=m} \int_{\mathbb{R}_{+}^{n}}\left\langle A_{\alpha \beta}(x) D^{\beta} u(x), D^{\alpha} v(x)\right\rangle d x
$$


where the coefficient matrices $A_{\alpha \beta}$ are given by

$$
A_{\alpha \beta}=\operatorname{det} \lambda^{\prime} \sum_{|\gamma|=|\tau|=m} P_{\alpha \beta}^{\gamma \tau}\left(\varkappa^{\prime} \circ \lambda\right)\left(\mathfrak{A}_{\gamma \tau} \circ \lambda\right)
$$

for some scalar homogeneous polynomials $P_{\alpha \beta}^{\gamma \tau}$ of the elements of the matrix $\varkappa^{\prime}(\lambda(x))$ with $\operatorname{deg} P_{\alpha \beta}^{\gamma \tau}=$ $2 m$. In view of (5.5)-(5.15),

$$
\sum_{|\alpha|=|\beta|=m}\left[A_{\alpha \beta}\right]_{\mathrm{BMO}\left(\mathbb{R}_{+}^{n}\right)} \leq c\left(\kappa^{-1}[\nabla \varphi]_{\mathrm{BMO}\left(\mathbb{R}^{n-1}\right)}+\sum_{|\alpha|=|\beta|=m}\left[\mathfrak{A}_{\alpha \beta}\right]_{\operatorname{BMO}(G)}\right),
$$

where $c$ depends on $n, m$, and $\|\nabla \varphi\|_{L_{\infty}\left(\mathbb{R}^{n-1}\right)}$.

By (5.38)

$$
\left|L(u, u)-L_{0}(u, u)\right| \leq c \delta\|u\|_{V_{2}^{m, 0}\left(\mathbb{R}_{+}^{n}\right)}^{2}
$$

and, therefore,

$$
\Re L_{0}(u, u) \geq \Re \mathcal{L}(\mathcal{U}, \mathcal{U})-c \delta\|u\|_{V_{2}^{m, 0}\left(\mathbb{R}_{+}^{n}\right)}^{2} .
$$

Using (5.28) and the equivalence

$$
\|\mathcal{U}\|_{V_{2}^{m, 0}(G)} \sim\|u\|_{V_{2}^{m, 0}\left(\mathbb{R}_{+}^{n}\right)}
$$

(cf. the discussion in §5.3), we arrive at (4.6). Thus, all conditions of Lemma 4.1 hold and the result follows. The improvement of (5.30) for $m=1$ mentioned in the statement (i) holds because in this case $L=L_{0}$.

\section{Dirichlet problem in a bounded Lipschitz domain}

\subsection{Preliminaries}

Let $\Omega$ be a bounded Lipschitz domain in $\mathbb{R}^{n}$ which means (cf. [47], p. 189) that there exists a finite open covering $\left\{\mathcal{O}_{j}\right\}_{1 \leq j \leq N}$ of $\partial \Omega$ with the property that, for every $j \in\{1, \ldots, N\}, \mathcal{O}_{j} \cap \Omega$ coincides with the portion of $\mathcal{O}_{j}$ lying in the over-graph of a Lipschitz function $\varphi_{j}: \mathbb{R}^{n-1} \rightarrow \mathbb{R}$ (where $\mathbb{R}^{n-1} \times \mathbb{R}$ is a new system of coordinates obtained from the original one via a rigid motion). We then define the Lipschitz constant of a bounded Lipschitz domain $\Omega \subset \mathbb{R}^{n}$ as

$$
\inf \left(\max \left\{\left\|\nabla \varphi_{j}\right\|_{L_{\infty}\left(\mathbb{R}^{n-1}\right)}: 1 \leq j \leq N\right\}\right)
$$

where the infimum is taken over all possible families $\left\{\varphi_{j}\right\}_{1 \leq j \leq N}$ as above.

It is a classical result that the surface measure $d \sigma$ is well-defined and that there exists an outward pointing normal vector $\nu$ at almost every point on $\partial \Omega$.

We denote by $\rho(X)$ the distance from $X \in \mathbb{R}^{n}$ to $\partial \Omega$ and, for $p, a$ and $m$ as in (4.1), introduce the weighted Sobolev space $V_{p}^{m, a}(\Omega)$ naturally associated with the norm

$$
\|\mathcal{U}\|_{V_{p}^{m, a}(\Omega)}:=\left(\sum_{0 \leq|\beta| \leq m} \int_{\Omega}\left|\rho(X)^{|\beta|-m} D^{\beta} \mathcal{U}(X)\right|^{p} \rho(X)^{p a} d X\right)^{1 / p} .
$$

One can check the equivalence of the norms 


$$
\|\mathcal{U}\|_{V_{p}^{m, a}(\Omega)} \sim\left\|\rho_{\text {reg }}^{a} \mathcal{U}\right\|_{V_{p}^{m, 0}(\Omega)},
$$

where $\rho_{\text {reg }}(X)$ stands for the regularized distance from $X$ to $\partial \Omega$ (in the sense of Theorem 2, p. 171 in [47]). It is also easily proved that $C_{0}^{\infty}(\Omega)$ is dense in $V_{p}^{m, a}(\Omega)$ and that

$$
\|\mathcal{U}\|_{V_{p}^{m, a}(\Omega)} \sim\left(\sum_{|\beta|=m} \int_{\Omega}\left|D^{\beta} \mathcal{U}(X)\right|^{p} \rho(X)^{p a} d X\right)^{1 / p}
$$

uniformly for $\mathcal{U} \in C_{0}^{\infty}(\Omega)$. As in (5.24), we set

$$
V_{p}^{-m, a}(\Omega):=\left(V_{p^{\prime}}^{m,-a}(\Omega)\right)^{*} .
$$

Let us fix a Cartesian coordinates system and consider the differential operator

$$
\mathcal{A U}=\mathcal{A}\left(X, D_{X}\right) \mathcal{U}:=\sum_{|\alpha|=|\beta|=m} D^{\alpha}\left(\mathcal{A}_{\alpha \beta}(X) D^{\beta} \mathcal{U}\right), \quad X \in \Omega,
$$

with measurable $l \times l$ matrix-valued coefficients. The corresponding sesquilinear form will be denoted by $\mathcal{A}(\mathcal{U}, \mathcal{V})$. Similarly to (5.26) and (5.28) we impose the conditions

$$
\sum_{|\alpha|=|\beta|=m}\left\|\mathcal{A}_{\alpha \beta}\right\|_{L_{\infty}(\Omega)} \leq \kappa^{-1}
$$

and

$$
\Re \mathcal{A}(\mathcal{U}, \mathcal{U}) \geq \kappa \sum_{|\gamma|=m}\left\|D^{\gamma} \mathcal{U}\right\|_{L_{2}(\Omega)}^{2} \quad \text { for all } \mathcal{U} \in V_{2}^{m, 0}(G)
$$

\subsection{Interior regularity of solutions}

Lemma 6.1 Let $\Omega \subset \mathbb{R}^{n}$ be a bounded Lipschitz domain. Pick two functions $\mathcal{H}, \mathcal{Z} \in C_{0}^{\infty}(\Omega)$ such that $\mathcal{H} \mathcal{Z}=\mathcal{H}$, and assume that

$$
\sum_{|\alpha|=|\beta|=m}\left[\mathcal{A}_{\alpha \beta}\right]_{\mathrm{BMO}(\Omega)} \leq \delta
$$

where

$$
\delta \leq \frac{c(m, n, \kappa)}{p p^{\prime}}
$$

with a sufficiently small constant $c(m, n, \kappa)>0$.

If $\mathcal{U} \in W_{q}^{m}(\Omega$, loc $)$ for a certain $q<p$ and $\mathcal{A U} \in W_{p}^{-m}(\Omega$, loc $)$, then $\mathcal{U} \in W_{p}^{m}(\Omega$, loc $)$ and

$$
\|\mathcal{H} \mathcal{U}\|_{W_{p}^{m}(\Omega)} \leq C\left(\|\mathcal{H} \mathcal{A}(\cdot, D) \mathcal{U}\|_{W_{p}^{-m}(\Omega)}+\|\mathcal{Z} \mathcal{U}\|_{W_{q}^{m}(\Omega)}\right)
$$

Proof. We start with a trick applied in [10] under slightly different circumstances. We shall use the notation $\mathcal{A}_{Y}$ for the operator $\mathcal{A}\left(Y, D_{X}\right)$, where $Y \in \Omega$ and the notation $\Phi_{Y}$ for a fundamental solution of $\mathcal{A}_{Y}$ in $\mathbb{R}^{n}$. Then, with star denoting the convolution product,

$$
\mathcal{H} \mathcal{U}+\Phi_{Y} *\left(\mathcal{A}-\mathcal{A}_{Y}\right)(\mathcal{H} \mathcal{U})=\Phi_{Y} *(\mathcal{H} \mathcal{A} \mathcal{U})+\Phi_{Y} *([\mathcal{A}, \mathcal{H}](\mathcal{Z U}))
$$


and, consequently, for each multi-index $\gamma,|\gamma|=m$,

$$
\begin{gathered}
D^{\gamma}(\mathcal{H} \mathcal{U})+\sum_{|\alpha|=|\beta|=m} D^{\alpha+\gamma} \Phi_{Y} *\left(\left(\mathcal{A}_{\alpha \beta}-\mathcal{A}_{\alpha \beta}(Y)\right) D^{\beta}(\mathcal{H} \mathcal{U})\right) \\
=D^{\gamma} \Phi_{Y} *(\mathcal{H} \mathcal{A U})+D^{\gamma} \Phi_{Y} *([\mathcal{A}, \mathcal{H}](\mathcal{Z U})) .
\end{gathered}
$$

Writing this equation at the point $Y$ and using (3.32), we obtain

$$
\begin{aligned}
& \left(1-C p p^{\prime} \delta\right) \sum_{|\gamma|=m}\left\|D^{\gamma}(\mathcal{H} \mathcal{U})\right\|_{L_{p}(\Omega)} \\
& \quad \leq C(p, \kappa)\left(\|\mathcal{H} \mathcal{A} \mathcal{U}\|_{W_{p}^{-m}(\Omega)}+\|[\mathcal{A}, \mathcal{H}](\mathcal{Z U})\|_{W_{p}^{-m}(\Omega)}\right)
\end{aligned}
$$

Let $p^{\prime}<n$. We have for every $\mathcal{V} \in \stackrel{\circ}{W}_{p}^{m}(\Omega)$

$$
\begin{aligned}
& \left|\int_{\Omega}\langle[\mathcal{A}, \mathcal{H}](\mathcal{Z U}), \mathcal{V}\rangle d X\right|=|\mathcal{A}(\mathcal{H} \mathcal{Z} \mathcal{U}, \mathcal{V})-\mathcal{A}(\mathcal{Z U}, \mathcal{H} \mathcal{V})| \\
& \quad \leq c\left(\|\mathcal{Z} \mathcal{U}\|_{W_{p}^{m-1}(\Omega)}\|\mathcal{V}\|_{W_{p^{\prime}}^{m}(\Omega)}+\|\mathcal{Z U}\|_{W_{\frac{p n}{n+p}}^{m}(\Omega)}\|\mathcal{V}\|_{W_{\frac{p^{\prime} n}{n-p^{\prime}}}^{m-1}(\Omega)}\right)
\end{aligned}
$$

By Sobolev's theorem

$$
\|\mathcal{Z U}\|_{W_{p}^{m-1}(\Omega)} \leq c\|\mathcal{Z} \mathcal{U}\|_{W_{\frac{p}{n+p}}^{m}}(\Omega)
$$

and

$$
\|\mathcal{V}\|_{\substack{W_{p^{\prime} n}^{m-1}(\Omega) \\ n-p^{\prime}}} \leq c\|\mathcal{V}\|_{W_{p^{\prime}}^{m}(\Omega)}
$$

Therefore,

$$
\left|\int_{\Omega}\langle[\mathcal{A}, \mathcal{H}](\mathcal{Z U}), \mathcal{V}\rangle d X\right| \leq c\|\mathcal{Z} \mathcal{U}\|_{W_{\frac{p n}{n+p}}^{m}(\Omega)}\|\mathcal{V}\|_{W_{p^{\prime}}^{m}(\Omega)}
$$

which is equivalent to the inequality

$$
\|[\mathcal{A}, \mathcal{H}](\mathcal{Z U})\|_{W_{p}^{-m}(\Omega)} \leq c\|\mathcal{Z U}\|_{W_{\frac{p n}{n+p}}^{m}(\Omega)} .
$$

In the case $p^{\prime} \geq n$, the same argument leads to a similar inequality, where $p n /(n+p)$ is replaced by $1+\varepsilon$ with an arbitrary $\varepsilon>0$ for $p^{\prime}>n$ and $\varepsilon=0$ for $p^{\prime}=n$.

Now, (6.11) follows from (6.14) if $p^{\prime} \geq n$ and $p^{\prime}<n, q \geq p n /(n+p)$. In the remaining case the goal is achieved by iterating this argument finitely many times.

Corollary 6.2 Let $p \geq 2$ and suppose that (6.9) and (6.10) hold. If $\mathcal{U} \in W_{2}^{m}(\Omega$, loc) and $\mathcal{A U} \in$ $W_{p}^{-m}(\Omega$, loc $)$, then $\mathcal{U} \in W_{p}^{m}(\Omega$, loc $)$ and

$$
\|\mathcal{H} \mathcal{U}\|_{W_{p}^{m}(\Omega)} \leq C\left(\|\mathcal{Z} \mathcal{A}(\cdot, D) \mathcal{U}\|_{W_{p}^{-m}(\Omega)}+\|\mathcal{Z} \mathcal{U}\|_{W_{2}^{m-1}(\Omega)}\right)
$$


Proof. Let $\mathcal{Z}_{0}$ denote a real-valued function in $C_{0}^{\infty}(\Omega)$ such that $\mathcal{H} \mathcal{Z}_{0}=\mathcal{H}$ and $\mathcal{Z}_{0} \mathcal{Z}=\mathcal{Z}_{0}$. By (6.11)

$$
\|\mathcal{H} \mathcal{U}\|_{W_{p}^{m}(\Omega)} \leq C\left(\|\mathcal{H} \mathcal{A}(\cdot, D) \mathcal{U}\|_{W_{p}^{-m}(\Omega)}+\left\|\mathcal{Z}_{0} \mathcal{U}\right\|_{W_{2}^{m}(\Omega)}\right)
$$

and it follows from (6.8) that

$$
\left\|\mathcal{Z}_{0} \mathcal{U}\right\|_{W_{2}^{m}(\Omega)}^{2} \leq c \kappa^{-1} \Re \mathcal{A}\left(\mathcal{Z}_{0} \mathcal{U}, \mathcal{Z}_{0} \mathcal{U}\right)
$$

Furthermore,

$$
\left|\mathcal{A}\left(\mathcal{Z}_{0} \mathcal{U}, \mathcal{Z}_{0} \mathcal{U}\right)-\mathcal{A}\left(\mathcal{U}, \mathcal{Z}_{0}^{2} \mathcal{U}\right)\right| \leq c \kappa^{-1}\|\mathcal{Z} \mathcal{U}\|_{W_{2}^{m-1}(\Omega)}\left\|\mathcal{Z}_{0} \mathcal{U}\right\|_{W_{2}^{m}(\Omega)}
$$

Hence

$$
\left\|\mathcal{Z}_{0} \mathcal{U}\right\|_{W_{2}^{m}(\Omega)}^{2} \leq c \kappa^{-1}\left(\|\mathcal{Z} \mathcal{A} \mathcal{U}\|_{W_{2}^{-m}(\Omega)}\left\|\mathcal{Z}_{0}^{2} \mathcal{U}\right\|_{W_{2}^{m}(\Omega)}+\kappa^{-1}\|\mathcal{Z} \mathcal{U}\|_{W_{2}^{m-1}(\Omega)}\left\|\mathcal{Z}_{0} \mathcal{U}\right\|_{W_{2}^{m}(\Omega)}\right)
$$

and, therefore,

$$
\left\|\mathcal{Z}_{0} \mathcal{U}\right\|_{W_{2}^{m}(\Omega)} \leq c \kappa^{-1}\left(\|\mathcal{Z} \mathcal{A} \mathcal{U}\|_{W_{2}^{-m}(\Omega)}+\kappa^{-1}\|\mathcal{Z} \mathcal{U}\|_{W_{2}^{m-1}(\Omega)}\right) .
$$

Combining this inequality with (6.21) we arrive at (6.20).

\subsection{Invertibility of $\mathcal{A}: V_{p}^{m, a}(\Omega) \longrightarrow V_{p}^{-m, a}(\Omega)$}

Recall the infinitesimal mean oscillations as defined in (1.10).

Theorem 6.3 Let $1<p<\infty, 0<s<1$, and $a=1-s-1 / p$. Furthermore, let $\Omega$ be a bounded Lipschitz domain in $\mathbb{R}^{n}$. Suppose that the differential operator $\mathcal{A}$ is as in $\S 6.1$ and that, in addition,

$$
\sum_{|\alpha|=|\beta|=m}\left\{\mathcal{A}_{\alpha \beta}\right\}_{\operatorname{Osc}(\Omega)}+\{\nu\}_{\operatorname{Osc}(\partial \Omega)} \leq \delta,
$$

where

$$
\left(p p^{\prime}+\frac{1}{s(1-s)}\right) \frac{\delta}{s(1-s)} \leq c
$$

for a sufficiently small constant $c>0$ independent of $p$ and $s$. In the case $m=1$ the factor $\delta / s(1-s)$ in (6.27) can be replaced by $\delta$.

Then the operator

$$
\mathcal{A}: V_{p}^{m, a}(\Omega) \longrightarrow V_{p}^{-m, a}(\Omega)
$$

is an isomorphism.

Proof. We shall proceed in a series a steps starting with

(i) The construction of the auxiliary domain $G$ and operator $\mathcal{L}$.

Let $\varepsilon$ be small enough so that 


$$
\sum_{|\alpha|=|\beta|=m} f_{B_{r} \cap \Omega} f_{B_{r} \cap \Omega}\left|\mathcal{A}_{\alpha \beta}(X)-\mathcal{A}_{\alpha \beta}(Y)\right| d X d Y \leq 2 \delta
$$

for all balls in $\left\{B_{r}\right\}_{\Omega}$ with radii $r<\varepsilon$ and

$$
f_{B_{r} \cap \partial \Omega} f_{B_{r} \cap \partial \Omega}|\nu(X)-\nu(Y)| d \sigma_{X} d \sigma_{Y} \leq 2 \delta
$$

for all balls in $\left\{B_{r}\right\}_{\partial \Omega}$ with radii $r<\varepsilon$.

We fix a ball $B_{\varepsilon}$ in $\left\{B_{\varepsilon}\right\}_{\partial \Omega}$ and assume without loss of generality that, in a suitable system of Cartesian coordinates,

$$
\Omega \cap B_{\varepsilon}=\left\{X=\left(X^{\prime}, X_{n}\right) \in B_{\varepsilon}: X_{n}>\varphi\left(X^{\prime}\right)\right\}
$$

for some Lipschitz function $\varphi: \mathbb{R}^{n-1} \rightarrow \mathbb{R}$. Consider now the unique cube $Q(\varepsilon)$ (relative to this system of coordinates) which is inscribed in $B_{\varepsilon}$ and denote its projection onto $\mathbb{R}^{n-1}$ by $Q^{\prime}(\varepsilon)$. Since $\nabla \varphi=-\nu^{\prime} / \nu_{n}$, it follows from (6.30) that

$$
f_{B_{r}^{\prime}} f_{B_{r}^{\prime}}\left|\nabla \varphi\left(X^{\prime}\right)-\nabla \varphi\left(Y^{\prime}\right)\right| d X^{\prime} d Y^{\prime} \leq c(n) \delta
$$

where $B_{r}^{\prime}=B_{r} \cap \mathbb{R}^{n-1}, r<\varepsilon$. Let us retain the notation $\varphi$ for the mirror extension of the function $\varphi$ from $Q^{\prime}(\varepsilon)$ onto $\mathbb{R}^{n-1}$.

We extend $\mathcal{A}_{\alpha \beta}$ from $Q(\varepsilon) \cap \Omega$ onto $Q(\varepsilon) \backslash \Omega$ by setting

$$
\mathcal{A}_{\alpha \beta}(X):=\mathcal{A}_{\alpha \beta}\left(X^{\prime},-X_{n}+2 \varphi\left(X^{\prime}\right)\right), \quad X \in Q(\varepsilon) \backslash \Omega,
$$

and we shall use the notation $\mathfrak{A}_{\alpha \beta}$ for the periodic extension of $\mathcal{A}_{\alpha \beta}$ from $Q(\varepsilon)$ onto $\mathbb{R}^{n}$.

Consistent with the earlier discussion in $\S 5$, we shall denote the special Lipschitz domain $\{X=$ $\left.\left(X^{\prime}, X_{n}\right): X^{\prime} \in \mathbb{R}^{n-1}, X_{n}>\varphi\left(X^{\prime}\right)\right\}$ by $G$. One can easily see that, owing to $2 \varepsilon n^{-1 / 2}$-periodicity of $\varphi$ and $\mathcal{A}_{\alpha \beta}$,

$$
\sum_{|\alpha|=|\beta|=m}\left[\mathcal{A}_{\alpha \beta}\right]_{\mathrm{BMO}(G)}+[\nabla \varphi]_{\mathrm{BMO}\left(\mathbb{R}^{n-1}\right)} \leq c(n) \delta
$$

Now, with the operator $\mathcal{A}\left(X, D_{X}\right)$ in $\Omega$, we associate an auxiliary operator $\mathcal{L}\left(X, D_{X}\right)$ in $G$ given by (5.25).

(ii) Uniqueness.

Assuming that $\mathcal{U} \in V_{p}^{m, a}(\Omega)$ satisfies $\mathcal{L U}=0$ in $\Omega$, we shall show that $\mathcal{U} \in V_{2}^{m, 0}(\Omega)$. This will imply that $\mathcal{U}=0$ which proves the injectivity of the operator (6.28).

To this end, pick a function $\mathcal{H} \in C_{0}^{\infty}(Q(\varepsilon))$ and write $\mathcal{L}(\mathcal{H} \mathcal{U})=[\mathcal{L}, \mathcal{H}] \mathcal{U}$. Also, fix a small $\theta>0$ and select a smooth function $\Lambda$ on $\mathbb{R}_{+}^{1}$, which is identically 1 on $[0,1]$ and which vanishes identically on $(2, \infty)$. Then by (ii) in Lemma 5.1

$$
\mathcal{L}(\mathcal{H} \mathcal{U})-[\mathcal{L}, \mathcal{H}]\left(\Lambda\left(\rho_{\text {reg }} / \theta\right) \mathcal{U}\right) \in V_{2}^{-m, 0}(G) \cap V_{p}^{-m, a}(G) .
$$

Note that the operator

$$
[\mathcal{L}, \mathcal{H}] \rho_{\text {reg }}^{-1}: V_{p}^{m, a}(G) \longrightarrow V_{p}^{-m, a}(G)
$$


is bounded and that the norm of the multiplier $\rho_{\text {reg }} \Lambda\left(\rho_{\text {reg }} / \theta\right)$ in $V_{p}^{m, a}(G)$ is $O(\theta)$. Moreover, the same is true for $p=2$ and $a=0$.

The inclusion (6.35) can be written in the form

$$
\mathcal{L}(\mathcal{H} \mathcal{U})+\mathcal{M}(\mathcal{Z U}) \in V_{p}^{-m, a}(G) \cap V_{2}^{-m, 0}(G),
$$

where $\mathcal{Z} \in C_{0}^{\infty}\left(\mathbb{R}^{n}\right), \mathcal{Z} \mathcal{H}=\mathcal{H}$ and $\mathcal{M}$ is a linear operator mapping

$$
V_{p}^{m, a}(G) \rightarrow V_{p}^{-m, a}(G) \quad \text { and } \quad V_{2}^{m, 0}(G) \rightarrow V_{2}^{-m, 0}(G)
$$

with both norms of order $O(\theta)$.

Select a finite covering of $\bar{\Omega}$ by cubes $Q_{j}(\varepsilon)$ and let $\left\{\mathcal{H}_{j}\right\}$ be a smooth partition of unity subordinate to $\left\{Q_{j}(\varepsilon)\right\}$. Also, let $\mathcal{Z}_{j} \in C_{0}^{\infty}\left(Q_{j}(\varepsilon)\right)$ be such that $\mathcal{H}_{j} \mathcal{Z}_{j}=\mathcal{H}_{j}$. By $G_{j}$ we denote the special Lipschitz domain generated by the cube $Q_{j}(\varepsilon)$ as in part (i) of the present proof. The corresponding operators $\mathcal{L}$ and $\mathcal{M}$ will be denoted by $\mathcal{L}_{j}$ and $\mathcal{M}_{j}$, respectively. It follows from (6.37) that

$$
\mathcal{H}_{j} \mathcal{U}+\sum_{k}\left(\mathcal{L}_{j}^{-1} \mathcal{M}_{j} \mathcal{Z}_{j} \mathcal{Z}_{k}\right)\left(\mathcal{H}_{k} \mathcal{U}\right) \in V_{p}^{m, a}(\Omega) \cap V_{2}^{m, 0}(\Omega)
$$

Taking into account that the norms of the matrix operator $\mathcal{L}_{j} \mathcal{M}_{j} \mathcal{Z}_{j} \mathcal{Z}_{k}$ in the spaces $V_{p}^{m, a}(\Omega)$ and $V_{2}^{m, 0}(\Omega)$ are $O(\theta)$, we may take $\theta>0$ small enough and obtain $\mathcal{H}_{j} \mathcal{U} \in V_{2}^{m, 0}(\Omega)$, i.e. $\mathcal{U} \in V_{2}^{m, 0}(\Omega)$. Therefore, $\mathcal{L}: V_{p}^{m, a}(\Omega) \rightarrow V_{p}^{-m, a}(\Omega)$ is injective.

(iii) A priori estimate.

Let $p \geq 2$ and assume that $\mathcal{U} \in V_{p}^{m, a}(\Omega)$. Referring to Corollary 6.2 and arguing as in part (ii) of the present proof, we arrive at the equation

$$
\mathcal{H}_{j} \mathcal{U}+\sum_{k}\left(\mathcal{L}_{j}^{-1} \mathcal{M}_{j} \mathcal{Z}_{j} \mathcal{Z}_{k}\right)\left(\mathcal{H}_{k} \mathcal{U}\right)=\mathcal{F}
$$

whose right-hand side satisfies

$$
\|\mathcal{F}\|_{V_{p}^{m, a}(\Omega)} \leq c\left(\|\mathcal{A} \mathcal{U}\|_{V_{p}^{-m, a}(\Omega)}+\|\mathcal{U}\|_{W_{2}^{m-1}(\omega)}\right),
$$

for some domain $\omega$ with $\bar{\omega} \subset \Omega$. Since the $V_{p}^{m, a}(\Omega)$-norm of the sum in (6.40) does not exceed $C \theta\|\mathcal{U}\|_{V_{p}^{m, a}(\Omega)}$, we obtain the estimate

$$
\|\mathcal{U}\|_{V_{p}^{m, a}(\Omega)} \leq c\left(\|\mathcal{A} \mathcal{U}\|_{V_{p}^{-m, a}(\Omega)}+\|\mathcal{U}\|_{W_{2}^{m-1}(\omega)}\right)
$$

(iv) End of proof.

Let $p \geq 2$. The range of the operator $\mathcal{A}: V_{p}^{m, a}(\Omega) \rightarrow V_{p}^{-m, a}(\Omega)$ is closed by (6.40) and the compactness of the restriction operator: $V_{p}^{m, a}(\Omega) \rightarrow W_{2}^{m-1}(\omega)$. Since the coefficients of the adjoint operator $\mathcal{L}^{*}$ satisfy the same conditions as those of $\mathcal{L}$, the operator $\mathcal{L}^{*}: V_{p^{\prime}}^{m, a}(\Omega) \rightarrow V_{p^{\prime}}^{-m,-a}(\Omega)$ is injective. Therefore, we conclude that $\mathcal{L}: V_{p}^{m, a}(\Omega) \rightarrow V_{p}^{-m,-a}(\Omega)$ is surjective. Being also injective, $\mathcal{L}$ is isomorphic if $p \geq 2$. Hence $\mathcal{L}^{*}$ is isomorphic for $p^{\prime} \leq 2$. This means that $\mathcal{L}$ is isomorphic for $p \leq 2$. The result follows. 


\subsection{Traces and extensions}

Let $\Omega \subset \mathbb{R}^{n}$ be a bounded Lipschitz domain and, for $m \in \mathbb{N}$ as well as $1<p<\infty$ and $-1 / p<a<$ $1-1 / p$, consider a new space, $W_{p}^{m, a}(\Omega)$, consisting of functions $\mathcal{U} \in L_{p}(\Omega, l o c)$ with the property that $\rho^{a} D^{\alpha} \mathcal{U} \in L_{p}(\Omega)$ for all multi-indices $\alpha$ with $|\alpha|=m$. We equip $W_{p}^{m, a}(\Omega)$ with the norm

$$
\|\mathcal{U}\|_{W_{p}^{m, a}(\Omega)}:=\sum_{|\alpha|=m}\left\|D^{\alpha} \mathcal{U}\right\|_{L_{p}\left(\Omega, \rho(X)^{a p} d X\right)}+\|\mathcal{U}\|_{L_{p}(\omega)}
$$

where $\omega$ is an open non-empty domain, $\bar{\omega} \subset \Omega$. An equivalent norm is given by the expression in (1.6). We omit the standard proof of the fact that

$$
C^{\infty}(\bar{\Omega}) \hookrightarrow W_{p}^{m, a}(\Omega) \text { densely. }
$$

Recall that for $p \in(1, \infty)$ and $s \in(0,1)$ the Besov space $B_{p}^{s}(\partial \Omega)$ is then defined via the requirement (1.7). If we introduce the $L_{p}$-modulus of continuity

$$
\omega_{p}(f, t):=\left(\int_{\substack{|X-Y|<t \\ X, Y \in \partial \Omega}}|f(X)-f(Y)|^{p} d \sigma_{X} d \sigma_{Y}\right)^{1 / p}
$$

then

$$
\|f\|_{B_{p}^{s}(\partial \Omega)} \sim\|f\|_{L_{p}(\partial \Omega)}+\left(\int_{0}^{\infty} \frac{\omega_{p}(f, t)^{p}}{t^{n-1+p s}} d t\right)^{1 / p},
$$

uniformly for $f \in B_{p}^{s}(\partial \Omega)$.

The nature of our problem requires that we work with Besov spaces (defined on Lipschitz boundaries) which exhibit a higher order of smoothness. In accordance with [26], we now make the following definition.

Definition 6.1 For $p \in(1, \infty), m \in \mathbb{N}$ and $s \in(0,1)$, define the (higher order) Besov space $\dot{B}_{p}^{m-1+s}(\partial \Omega)$ as the collection of all finite families $\dot{f}=\left\{f_{\alpha}\right\}_{|\alpha| \leq m-1}$ of functions defined on $\partial \Omega$ with the following property. For each multi-index $\alpha$ of length $\leq m-1$ let

$$
R_{\alpha}(X, Y):=f_{\alpha}(X)-\sum_{|\beta| \leq m-1-|\alpha|} \frac{1}{\beta !} f_{\alpha+\beta}(Y)(X-Y)^{\beta}, \quad X, Y \in \partial \Omega,
$$

and consider the $L_{p}$-modulus of continuity

$$
r_{\alpha}(t):=\left(\int_{\substack{|X-Y|<t \\ X, Y \in \partial \Omega}}\left|R_{\alpha}(X, Y)\right|^{p} d \sigma_{X} d \sigma_{Y}\right)^{1 / p}
$$

Then

$$
\|\dot{f}\|_{\dot{B}_{p}^{m-1+s}(\partial \Omega)}:=\sum_{|\alpha| \leq m-1}\left\|f_{\alpha}\right\|_{L_{p}(\partial \Omega)}+\sum_{|\alpha| \leq m-1}\left(\int_{0}^{\infty} \frac{r_{\alpha}(t)^{p}}{t^{p(m-1+s-|\alpha|)+n-1}} d t\right)^{1 / p}<\infty
$$


For further reference we note here that for each fixed $\kappa>0$, an equivalent norm is obtained by replacing $r_{\alpha}(t)$ by $r_{\alpha}(\kappa t)$ in (6.49). Also, when $m=1$, the above definition agrees with (1.7), thanks to (6.46).

A few notational conventions which make the exposition more transparent are as follows. Given a family of functions $\left\{f_{\alpha}\right\}_{|\alpha| \leq m-1}$ on $\partial \Omega$ and $X \in \Omega, Y, Z \in \partial \Omega$, set

$$
\begin{aligned}
& P_{\alpha}(X, Y):=\sum_{|\beta| \leq m-1-|\alpha|} \frac{1}{\beta !} f_{\alpha+\beta}(Y)(X-Y)^{\beta}, \quad \forall \alpha:|\alpha| \leq m-1, \\
& P(X, Y):=P_{(0, \ldots, 0)}(X, Y)
\end{aligned}
$$

so that

$$
R_{\alpha}(Y, Z)=f_{\alpha}(Y)-P_{\alpha}(Y, Z), \quad \forall \alpha:|\alpha| \leq m-1
$$

and the following elementary identities hold for each multi-index $\alpha$ of length $\leq m-1$ :

$$
\begin{aligned}
D_{X}^{\beta} P_{\alpha}(X, Y) & =P_{\alpha+\beta}(X, Y), \quad|\beta| \leq m-1-|\alpha|, \\
P_{\alpha}(X, Y)-P_{\alpha}(X, Z) & =\sum_{|\beta| \leq m-1-|\alpha|} R_{\alpha+\beta}(Y, Z) \frac{(X-Y)^{\beta}}{\beta !} .
\end{aligned}
$$

See, e.g., p. 177 in [47] for the last formula.

Lemma 6.4 For each $1<p<\infty,-1 / p<a<1-1 / p$ and $s=1-a-1 / p$, the trace operator

$$
\operatorname{Tr}: W_{p}^{1, a}(\Omega) \longrightarrow B_{p}^{s}(\partial \Omega)
$$

is well-defined, linear, bounded, onto and has $V_{p}^{1, a}(\Omega)$ as its null-space. Furthermore, there exists a linear, continuous mapping

$$
\mathcal{E}: B_{p}^{s}(\partial \Omega) \longrightarrow W_{p}^{1, a}(\Omega)
$$

called extension operator, such that $\operatorname{Tr} \circ \mathcal{E}=I$ (i.e., the operator 6.53) has a bounded, linear right-inverse).

Proof. By a standard argument involving a smooth partition of unity it suffices to deal with the case when $\Omega$ is the domain lying above the graph of a Lipschitz function $\varphi: \mathbb{R}^{n-1} \rightarrow \mathbb{R}$. Composing with the bi-Lipschitz homeomorphism $\mathbb{R}_{+}^{n} \ni\left(X^{\prime}, X_{n}\right) \mapsto\left(X^{\prime}, \varphi\left(X^{\prime}\right)+X_{n}\right) \in \Omega$ further reduces matters to the case when $\Omega=\mathbb{R}_{+}^{n}$, in which situation the claims in the lemma have been proved in [49].

We need to establish an analogue of Lemma 6.4 for higher smoothness spaces. While for $\Omega=\mathbb{R}_{+}^{n}$ this has been done by Uspenskiu in [49], the flattening argument used in Lemma 6.4 is no longer effective in this context. Let us also mention here that a result similar in spirit, valid for any Lipschitz domain $\Omega$ but with $B^{m-1+s+1 / p}(\Omega)$ in place of $W_{p}^{m, a}(\Omega)$ (cf. (1.16) for the relationship between these spaces) has been proved by A. Jonsson and H. Wallin in [26] (in fact, in this latter context, these authors have dealt with much more general sets than Lipschitz domains). The result which serves our purposes is as follows. 
Proposition 6.5 Let $1<p<\infty,-1 / p<a<1-1 / p, s=1-a-1 / p \in(0,1)$ and $m \in \mathbb{N}$. Define the higher order trace operator

$$
\operatorname{tr}_{m-1}: W_{p}^{m, a}(\Omega) \longrightarrow \dot{B}_{p}^{m-1+s}(\partial \Omega)
$$

by setting

$$
\operatorname{tr}_{m-1} \mathcal{U}:=\left\{i^{|\alpha|} \operatorname{Tr}\left[D^{\alpha} \mathcal{U}\right]\right\}_{|\alpha| \leq m-1},
$$

where the traces in the right-hand side are taken in the sense of Lemma 6.4. Then (6.55)-(6.56) is a a well-defined, linear, bounded operator, which is onto and has $V_{p}^{m, a}(\Omega)$ as its null-space. Moreover, it has a bounded, linear right-inverse, i.e. there exists a linear, continuous operator

$$
\mathcal{E}: \dot{B}_{p}^{m-1+s}(\partial \Omega) \longrightarrow W_{p}^{m, a}(\Omega)
$$

such that

$$
\dot{f}=\left\{f_{\alpha}\right\}_{|\alpha| \leq m-1} \in \dot{B}^{m-1+s}(\partial \Omega) \Rightarrow i^{|\alpha|} \operatorname{Tr}\left[D^{\alpha}(\mathcal{E} \dot{f})\right]=f_{\alpha}, \quad \forall \alpha:|\alpha| \leq m-1 .
$$

In order to facilitate the exposition, we isolate a couple of preliminary results prior to the proof of Proposition 6.5.

Lemma 6.6 Assume that $\varphi: \mathbb{R}^{n-1} \rightarrow \mathbb{R}$ is a Lipschitz function and define $\Phi: \mathbb{R}^{n-1} \rightarrow \partial \Omega \hookrightarrow \mathbb{R}^{n}$ by setting $\Phi\left(X^{\prime}\right):=\left(X^{\prime}, \varphi\left(X^{\prime}\right)\right)$ at each $X^{\prime} \in \mathbb{R}^{n-1}$. Define the Lipschitz domain $\Omega$ as $\{X=$ $\left.\left(X^{\prime}, X_{n}\right) \in \mathbb{R}^{n}: X_{n}>\varphi\left(X^{\prime}\right)\right\}$ and, for some fixed $m \in \mathbb{N}, p \in(1, \infty)$ and $s \in(0,1)$ consider a system of functions $f_{\alpha} \in B_{p}^{s}(\partial \Omega), \alpha \in \mathbb{N}_{0}^{n},|\alpha| \leq m-1$, with the property that

$$
\frac{\partial}{\partial X_{k}}\left[f_{\alpha}\left(\Phi\left(X^{\prime}\right)\right)\right]=\sum_{j=1}^{n} f_{\alpha+e_{j}}\left(\Phi\left(X^{\prime}\right)\right) \partial_{k} \Phi_{j}\left(X^{\prime}\right), \quad 1 \leq k \leq n-1,
$$

for each multi-index $\alpha$ of length $\leq m-2$, where $\left\{e_{j}\right\}_{j}$ is the canonical orthonormal basis in $\mathbb{R}^{n}$. Finally, for each $l \in\{1, \ldots, m-1\}$ introduce $\Delta_{l}:=\left\{\left(t_{1}, \ldots, t_{l}\right): 0 \leq t_{l} \leq \cdots \leq t_{1} \leq 1\right\}$, and define $R_{\alpha}(X, Y)$ as in [6.47). Then if $\alpha$ is an arbitrary multi-index of length $\leq m-2$ and $r:=m-1-|\alpha|$, the following identity holds:

$$
\begin{aligned}
R_{\alpha}\left(\Phi\left(X^{\prime}\right), \Phi\left(Y^{\prime}\right)\right) & \\
= & \sum_{\left(j_{1}, \ldots, j_{r}\right) \in\{1, \ldots, n\}^{r}}\left\{\int_{\Delta_{r}}\left[f_{\alpha+e_{j_{1}}+\cdots+e_{j_{r}}}\left(\Phi\left(Y^{\prime}+t_{r}\left(X^{\prime}-Y^{\prime}\right)\right)\right)-f_{\alpha+e_{j_{1}}+\cdots+e_{j_{r}}}\left(\Phi\left(Y^{\prime}\right)\right)\right]\right. \\
& \left.\times \prod_{k=1}^{r} \nabla \Phi_{j_{k}}\left(Y^{\prime}+t_{k}\left(X^{\prime}-Y^{\prime}\right)\right) \cdot\left(X^{\prime}-Y^{\prime}\right) d t_{r} \cdots d t_{1}\right\}, \quad X^{\prime}, Y^{\prime} \in \mathbb{R}^{n-1} .
\end{aligned}
$$

Proof. We shall show that for any system of functions $\left\{f_{\alpha}\right\}_{|\alpha| \leq m-1}$ which satisfies (6.59), any multi-index $\alpha \in \mathbb{N}_{0}^{n}$ with $|\alpha| \leq m-2$ and any $l \in \mathbb{N}$ with $l \leq r:=m-1-|\alpha|$, there holds 


$$
\begin{aligned}
f_{\alpha}\left(\Phi\left(X^{\prime}\right)\right)-\sum_{|\beta| \leq l} \frac{1}{\beta !} f_{\alpha+\beta}\left(\Phi\left(Y^{\prime}\right)\right)\left(\Phi\left(X^{\prime}\right)-\Phi\left(Y^{\prime}\right)\right)^{\beta} \\
=\sum_{\left(j_{1}, \ldots, j_{l}\right) \in\{1, \ldots, n\}^{l}}\left\{\int_{\Delta_{l}}\left[f_{\alpha+e_{j_{1}}+\cdots+e_{j_{l}}}\left(\Phi\left(Y^{\prime}+t_{l}\left(X^{\prime}-Y^{\prime}\right)\right)\right)-f_{\alpha+e_{j_{1}}+\cdots+e_{j_{l}}}\left(\Phi\left(Y^{\prime}\right)\right)\right]\right. \\
\left.\quad \times \prod_{k=1}^{l} \nabla \Phi_{j_{k}}\left(Y^{\prime}+t_{k}\left(X^{\prime}-Y^{\prime}\right)\right) \cdot\left(X^{\prime}-Y^{\prime}\right) d t_{l} \cdots d t_{1}\right\}
\end{aligned}
$$

Clearly, (6.60) follows from (6.47) and (6.61) by taking $l:=r$.

In order to justify (6.61) we proceed by induction on $l$. Concretely, when $l=1$ we may write, based on (6.59) and the Fundamental Theorem of Calculus,

$$
\begin{gathered}
f_{\alpha}\left(\Phi\left(X^{\prime}\right)\right)-f_{\alpha}\left(\Phi\left(Y^{\prime}\right)\right)-\sum_{j=1}^{n} f_{\alpha+e_{j}}\left(\Phi\left(Y^{\prime}\right)\right)\left(\Phi_{j}\left(X^{\prime}\right)-\Phi_{j}\left(Y^{\prime}\right)\right) \\
=\int_{0}^{1} \frac{d}{d t}\left[f_{\alpha}\left(\Phi\left(Y^{\prime}+t\left(X^{\prime}-Y^{\prime}\right)\right)\right)\right] d t \\
\quad-\sum_{j=1}^{n} f_{\alpha+e_{j}}\left(\Phi\left(Y^{\prime}\right)\right) \int_{0}^{1} \frac{d}{d t}\left[\Phi_{j}\left(Y^{\prime}+t\left(X^{\prime}-Y^{\prime}\right)\right)\right] d t \\
=\sum_{j=1}^{n}\left\{\int_{0}^{1}\left[f_{\alpha+e_{j}}\left(Y^{\prime}+t\left(X^{\prime}-Y^{\prime}\right)\right)-f_{\alpha+e_{j}}\left(\Phi\left(Y^{\prime}\right)\right)\right]\right. \\
\left.\times \nabla \Phi_{j}\left(Y^{\prime}+t\left(X^{\prime}-Y^{\prime}\right)\right) \cdot\left(X^{\prime}-Y^{\prime}\right) d t\right\}
\end{gathered}
$$

as wanted. To prove the version of (6.61) when $l$ is replaced by $l+1$ we split the sum in the left-hand side of (6.61), written for $l+1$ in place of $l$, according to whether $|\beta| \leq l$ or $|\beta|=l+1$ and denote the expressions created in this fashion by $S_{1}$ and $S_{2}$, respectively. Next, based on (6.59) and the Fundamental Theorem of Calculus, we write

$$
\begin{aligned}
& f_{\alpha+e_{j_{1}}+\cdots+e_{j_{l}}}\left(\Phi\left(Y^{\prime}+t_{l}\left(X^{\prime}-Y^{\prime}\right)\right)\right)-f_{\alpha+e_{j_{1}}+\cdots+e_{j_{l}}}\left(\Phi\left(Y^{\prime}\right)\right) \\
& =\sum_{i=1}^{n} \int_{0}^{t_{l}} f_{\alpha+e_{j_{1}}+\cdots+e_{j_{l}}+e_{i}}\left(\Phi\left(Y^{\prime}+t_{l+1}\left(X^{\prime}-Y^{\prime}\right)\right)\right) \nabla \Phi_{i}\left(Y^{\prime}+t_{l+1}\left(X^{\prime}-Y^{\prime}\right)\right) \cdot\left(X^{\prime}-Y^{\prime}\right) d t_{l+1}
\end{aligned}
$$

and use the induction hypothesis to conclude that

$$
\begin{gathered}
S_{1}=\sum_{\left(j_{1}, \ldots, j_{l+1}\right) \in\{1, \ldots, n\}^{l+1}}\left\{\int_{\Delta_{l+1}} f_{\alpha+e_{j_{1}}+\cdots+e_{j_{l+1}}}\left(\Phi\left(Y^{\prime}+t_{l+1}\left(X^{\prime}-Y^{\prime}\right)\right)\right)\right. \\
\left.\times \prod_{k=1}^{l+1} \nabla \Phi_{j_{k}}\left(Y^{\prime}+t_{k}\left(X^{\prime}-Y^{\prime}\right)\right) \cdot\left(X^{\prime}-Y^{\prime}\right) d t_{l+1} \cdots d t_{1}\right\}
\end{gathered}
$$


Thus, if

$$
F_{j}(t):=\Phi_{j}\left(Y^{\prime}+t\left(X^{\prime}-Y^{\prime}\right)\right)-\Phi_{j}\left(Y^{\prime}\right), \quad 1 \leq j \leq n,
$$

we may express $S_{1}$ in the form

$$
\begin{gathered}
S_{1}=\sum_{\left(j_{1}, \ldots, j_{l+1}\right) \in\{1, \ldots, n\}^{l+1}}\left\{\int_{\Delta_{l+1}}\left[f_{\alpha+e_{j_{1}}+\cdots+e_{j_{l+1}}}\left(\Phi\left(Y^{\prime}+t_{l+1}\left(X^{\prime}-Y^{\prime}\right)\right)\right)-f_{\alpha+e_{j_{1}}+\cdots+e_{j_{l+1}}}\left(\Phi\left(Y^{\prime}\right)\right)\right]\right. \\
\left.\times \prod_{k=1}^{l+1} \nabla \Phi_{j_{k}}\left(Y^{\prime}+t_{k}\left(X^{\prime}-Y^{\prime}\right)\right) \cdot\left(X^{\prime}-Y^{\prime}\right) d t_{l+1} \cdots d t_{1}\right\} \\
+\sum_{\left(j_{1}, \ldots, j_{l+1}\right) \in\{1, \ldots, n\}^{l+1}} f_{\alpha+e_{j_{1}}+\cdots+e_{j_{l+1}}}\left(\Phi\left(Y^{\prime}\right)\right) \int_{\Delta_{l+1}} \prod_{k=1}^{l+1} F_{j_{k}}^{\prime}\left(t_{k}\right) d t_{l+1} \cdots d t_{1} .
\end{gathered}
$$

Note that the first double sum above corresponds precisely to the expression in the right-hand side of (6.61) written with $l$ replaced by $l+1$. Our proof of (6.61) by induction is therefore complete as soon as we show that for each multi-index $\beta$ of length $l+1$,

$$
\sum_{\substack{\left(j_{1}, \ldots, j_{l+1}\right) \in\{1, \ldots, n\} \\ e_{1}+\cdots+e_{j_{l+1}}=\beta}} \int_{\Delta_{l+1}} \prod_{k=1}^{l+1} F_{j_{k}}^{\prime}\left(t_{k}\right) d t_{l+1} \cdots d t_{1}=\frac{1}{\beta !}\left(\Phi\left(X^{\prime}\right)-\Phi\left(Y^{\prime}\right)\right)^{\beta} .
$$

In turn, this is going to be a consequence of a general identity, to the effect that

$$
\sum_{\substack{\left(j_{1}, \ldots, j_{l}\right) \in\{1, \ldots, n\} \\ e_{j_{1}+\cdots+e_{j_{l}}=\beta}}} \int_{0}^{t_{0}} \int_{0}^{t_{1}} \cdots \int_{0}^{t_{l-1}} \prod_{k=1}^{l} F_{j_{k}}^{\prime}\left(t_{k}\right) d t_{l} \cdots d t_{1}=\frac{1}{\beta !} F\left(t_{0}\right)^{\beta},
$$

for any Lipschitz function $F=\left(F_{1}, \ldots, F_{n}\right):[0,1] \rightarrow \mathbb{C}^{n}$ with $F(0)=0$, any point $t_{0} \in[0,1]$ any $l \in \mathbb{N}$ and any multi-index $\beta$ of length $l$. Of course, the case most relevant for our purposes is when the $F_{j}$ 's are as in (6.65), $t_{0}=1$ and when $l$ is replaced by $l+1$, but the above formulation is best suited for proving (6.68) via induction on $l$. Indeed, the case $l=1$ is immediate from the Fundamental Theorem of Calculus and to pass from $l$ to $l+1$ it suffices to show that the two sides of (6.68) have the same derivative with respect to $t_{0}$. The important observation in carrying out the latter step is that the derivative of the left-hand side of (6.68) with respect to $t_{0}$ is an expression to which the current induction hypothesis is readily applicable. This justifies (6.68) and completes the proof of (6.60).

Corollary 6.7 Under the assumptions of Lemma [6.6. for each multi-index of length $\leq m-2$ the following estimate holds

$$
\left(\int_{0}^{\infty} \frac{r_{\alpha}(t)^{p}}{t^{p(m-1+s-|\alpha|)+n-1}} d t\right)^{1 / p} \leq C \sum_{|\gamma|=m-1}\left\|f_{\gamma}\right\|_{B_{p}^{s}(\partial \Omega)},
$$

where the constant $C$ depends only on $n, p, s$ and $\|\nabla \varphi\|_{L_{\infty}\left(\mathbb{R}^{n-1}\right)}$. 
Proof. The identity (6.60) gives

$$
\begin{aligned}
& \left|R_{\alpha}\left(\Phi\left(X^{\prime}\right), \Phi\left(Y^{\prime}\right)\right)\right| \\
& \quad \leq C\left|X^{\prime}-Y^{\prime}\right|^{m-1-|\alpha|} \sum_{|\gamma|=m-1} \int_{0}^{1}\left|f_{\gamma}\left(\Phi\left(Y^{\prime}+\tau\left(X^{\prime}-Y^{\prime}\right)\right)\right)-f_{\gamma}\left(\Phi\left(Y^{\prime}\right)\right)\right| d \tau
\end{aligned}
$$

for each $X^{\prime}, Y^{\prime} \in \mathbb{R}^{n-1}$, where the constant $C$ depends only on $n$ and $\|\nabla \Phi\|_{L_{\infty}}$ which, in turn, is controlled in terms of $\|\nabla \varphi\|_{L_{\infty}}$. Given an arbitrary $t>0$ we now integrate the $p$-th power of both sides in (6.70) for $X^{\prime}, Y^{\prime} \in \partial \mathbb{R}^{n-1}$ subject to $\left|\Phi\left(X^{\prime}\right)-\Phi\left(Y^{\prime}\right)\right|<t$. Using Fubini's Theorem and making the change of variables $Z^{\prime}:=Y^{\prime}+\tau\left(X^{\prime}-Y^{\prime}\right)$ we obtain, after noticing that $\left|Z^{\prime}-Y^{\prime}\right| \leq \tau t$,

$$
\begin{aligned}
r_{\alpha}(t)^{p} & \leq C t^{p(m-1-|\alpha|)} \sum_{|\gamma|=m-1} \int_{\substack{X^{\prime}, Y^{\prime} \in \mathbb{R}^{n-1} \\
\left|X^{\prime}-Y^{\prime}\right|<c t}} \int_{0}^{1}\left|f_{\gamma}\left(\Phi\left(Y^{\prime}+\tau\left(X^{\prime}-Y^{\prime}\right)\right)\right)-f_{\gamma}\left(\Phi\left(Y^{\prime}\right)\right)\right|^{p} d \tau d X^{\prime} d Y^{\prime} \\
& \leq C t^{p(m-1-|\alpha|)} \sum_{|\gamma|=m-1} \int_{0}^{1} \int_{\substack{Z^{\prime}, Y^{\prime} \in \mathbb{R}^{n-1} \\
\left|Z^{\prime}-Y^{\prime}\right|<c \tau t}}\left|f_{\gamma}\left(\Phi\left(Z^{\prime}\right)\right)-f_{\gamma}\left(\Phi\left(Y^{\prime}\right)\right)\right|^{p} d Z^{\prime} d Y^{\prime} d \tau \\
& \leq C t^{p(m-1+s-|\alpha|)+n-1} \sum_{|\gamma|=m-1} \int_{0}^{1} \frac{\omega_{p}\left(f_{\gamma}, c \tau t\right)^{p}}{\tau^{n-1} t^{p s+n-1}} d \tau .
\end{aligned}
$$

Consequently,

$$
\begin{aligned}
\int_{0}^{\infty} \frac{r_{\alpha}(t)^{p}}{t^{p(m-1+s-|\alpha|)+n-1}} d t & \leq C \sum_{|\gamma|=m-1} \int_{0}^{\infty} \int_{0}^{1} \frac{\omega_{p}\left(f_{\gamma}, c \tau t\right)^{p}}{\tau^{n-1} t^{p s+n-1}} d \tau d t \\
& \leq C \sum_{|\gamma|=m-1}\left(\int_{0}^{\infty} \frac{\omega_{p}\left(f_{\gamma}, r\right)^{p}}{r^{p s+n-1}} d r\right)\left(\int_{0}^{1} \frac{1}{\tau^{1-s p}} d \tau\right) \\
& \leq C \sum_{|\gamma|=m-1} \int_{0}^{\infty} \frac{\omega_{p}\left(f_{\gamma}, t\right)^{p}}{t^{p s+n-1}} d t
\end{aligned}
$$

after making the change of variables $r:=c \tau t$ in the second step. With this in hand, the estimate (6.69) follows by virtue of (6.46).

After this preamble, we are in a position to present the

Proof of Proposition 6.5. We divide the proof into a series of steps, starting with

Step I: The well-definiteness of trace. Let $\mathcal{U}$ be an arbitrary function in $W_{p}^{m, a}(\Omega)$ and set

$$
f_{\alpha}:=i^{|\alpha|} \operatorname{Tr}\left[D^{\alpha} \mathcal{U}\right], \quad \forall \alpha:|\alpha| \leq m-1 .
$$

It follows from Lemma 6.4 that these trace functions are well-defined and, in fact, 


$$
\sum_{|\alpha| \leq m-1}\left\|f_{\alpha}\right\|_{B_{p}^{s}(\partial \Omega)} \leq C\|\mathcal{U}\|_{W_{p}^{m, a}(\Omega)} .
$$

In order to prove that $\dot{f}:=\left\{f_{\alpha}\right\}_{|\alpha| \leq m-1}$ belongs to $\dot{B}_{p}^{m-1+s}(\partial \Omega)$, let $R_{\alpha}(X, Y)$ and $r_{\alpha}(t)$ be as in (6.47) -(6.48). Our goal is to show that for every multi-index $\alpha$ with $|\alpha| \leq m-1$,

$$
\left(\int_{0}^{\infty} \frac{r_{\alpha}(t)^{p}}{t^{p(m-1+s-|\alpha|)+n-1}} d t\right)^{1 / p} \leq C\|\mathcal{U}\|_{W_{p}^{m, a}(\Omega)} .
$$

To this end, we first observe that if $|\alpha|=m-1$ then the expression in the left-hand side of (6.75) is majorized by $C\left(\int_{0}^{\infty} \omega_{p}\left(f_{\alpha}, t\right)^{p} / t^{p s+n-1} d t\right)^{1 / p}$ which, by (6.46) and (6.74), is indeed $\leq$ $C\|\mathcal{U}\|_{W_{p}^{m, a}(\Omega)}$. To treat the case when $|\alpha|<m-1$ we assume that $\Omega$ is locally represented as $\{X$ : $\left.X_{n}>\varphi\left(X^{\prime}\right)\right\}$ for some Lipschitz function $\varphi: \mathbb{R}^{n-1} \rightarrow \mathbb{R}$ and, as before, set $\Phi\left(X^{\prime}\right):=\left(X^{\prime}, \varphi\left(X^{\prime}\right)\right)$, $X^{\prime} \in \mathbb{R}^{n-1}$. Then (6.59) holds, thanks to (6.73), for every multi-index $\alpha$ of length $\leq m-2$. Consequently, Corollary 6.7 applies and, in concert with (6.74), yields (6.75). This proves that the operator (6.55)-(6.56) is well-defined and bounded.

Step II: The extension operator. We introduce a co-boundary operator $\mathcal{E}$ which acts on $\dot{f}=$ $\left\{f_{\alpha}\right\}_{|\alpha| \leq m-1} \in \dot{B}_{p}^{m-1+s}(\partial \Omega)$ according to

$$
(\mathcal{E} \dot{f})(X)=\int_{\partial \Omega} \mathcal{K}(X, Y) P(X, Y) d \sigma_{Y}, \quad X \in \Omega,
$$

where $P(X, Y)$ is the polynomial associated with $\dot{f}$ as in (6.50). The integral kernel $\mathcal{K}$ is assumed to satisfy

$$
\begin{aligned}
& \int_{\partial \Omega} \mathcal{K}(X, Y) d \sigma_{Y}=1 \quad \text { for all } X \in \Omega, \\
& \left|D_{X}^{\alpha} \mathcal{K}(X, Y)\right| \leq c_{\alpha} \rho(X)^{1-n-|\alpha|}, \quad \forall X \in \Omega, \forall Y \in \partial \Omega,
\end{aligned}
$$

where $\alpha$ is an arbitrary multi-index, and

$$
\mathcal{K}(X, Y)=0 \quad \text { if }|X-Y| \geq 2 \rho(X) .
$$

One can take, for instance, the kernel

$$
\mathcal{K}(X, Y):=\eta\left(\frac{X-Y}{\varkappa \rho_{\mathrm{reg}}(X)}\right)\left(\int_{\partial \Omega} \eta\left(\frac{X-Z}{\varkappa \rho_{\mathrm{reg}}(X)}\right) d \sigma_{Z}\right)^{-1}
$$

where $\eta \in C_{0}^{\infty}\left(B_{2}\right), \eta=1$ on $B_{1}, \eta \geq 0$ and $\varkappa$ is a positive constant depending on the Lipschitz constant of $\partial \Omega$. Here, as before, $\rho_{\text {reg }}(X)$ stands for the regularized distance from $X$ to $\partial \Omega$.

For each $X \in \Omega$ and $Z \in \partial \Omega$ and for every multi-index $\gamma$ with $|\gamma|=m$ we then obtain

$$
D^{\gamma} \mathcal{E} \dot{f}(X)=\sum_{\substack{\alpha+\beta=\gamma \\|\alpha| \geq 1}} \frac{\gamma !}{\alpha ! \beta !} \int_{\partial \Omega} D_{X}^{\alpha} \mathcal{K}(X, Y)\left(P_{\beta}(X, Y)-P_{\beta}(X, Z)\right) d \sigma_{Y} .
$$

If for a fixed $\mu>1$ and for each $X \in \Omega$ and $t>0$ we set

$$
\Gamma_{t}:=\{Y \in \partial \Omega:|X-Y|<\mu t\}
$$


we may then estimate

$$
\begin{aligned}
\left|D^{\gamma} \mathcal{E} \dot{f}(X)\right|^{p} & \leq C \sum_{\substack{\alpha+\beta=\gamma \\
|\alpha| \geq 1}} \rho(X)^{-p|\alpha|} f_{\Gamma_{\rho(X)}}\left|P_{\beta}(X, Y)-P_{\beta}(X, Z)\right|^{p} d \sigma_{Y} \\
& \leq C \sum_{\substack{\alpha+\beta=\gamma \\
|\alpha| \geq 1}} \sum_{|\beta|+|\delta| \leq m-1} \rho(X)^{-p|\alpha|} f_{\Gamma_{\rho(X)}}\left|R_{\delta+\beta}(Y, Z)\right|^{p}|X-Y|^{p|\delta|} d \sigma_{Y}, \\
& \leq C \sum_{|\tau| \leq m-1} \rho(X)^{p(|\tau|-m)} f_{\Gamma_{\rho(X)}}\left|R_{\tau}(Y, Z)\right|^{p} d \sigma_{Y},
\end{aligned}
$$

where we have used Hölder's inequality and (6.52). Averaging the extreme terms in (6.83) for $Z$ in $\Gamma_{\rho(X)}$, we arrive at

$$
\left|D^{\gamma} \mathcal{E} \dot{f}(X)\right|^{p} \leq C \sum_{|\tau| \leq m-1} \rho(X)^{p(|\tau|-m)-2(n-1)} \int_{\Gamma_{\rho(X)}} \int_{\Gamma_{\rho(X)}}\left|R_{\tau}(Y, Z)\right|^{p} d \sigma_{Y} d \sigma_{Z}
$$

Consider now a Whitney decomposition of $\Omega$ into a family of dyadic cubes, $\left\{Q_{i}\right\}_{i \in \mathcal{I}}$. In particular, $l_{i}:=\operatorname{diam} Q_{i} \sim \operatorname{dist}\left(Q_{i}, \partial \Omega\right)$ uniformly for $i \in \mathcal{I}$. Thus, if $X \in Q_{i}$ for some $i \in I_{j}:=\{i \in \mathcal{I}$ : $\left.l_{i}=2^{-j}\right\}, j \in \mathbb{Z}$, the estimate (6.84) yields

$$
\left|D^{\gamma} \mathcal{E} \dot{f}(X)\right| \leq C \sum_{|\tau| \leq m-1} 2^{-j(|\tau|-m)}\left(2^{2 j(n-1)} \int_{\substack{Y, Z \in \partial \Omega \cap \varkappa Q_{i} \\|Y-Z|<\varkappa 2^{-j}}}\left|R_{\tau}(Y, Z)\right|^{p} d \sigma_{Y} d \sigma_{Z}\right)^{1 / p}
$$

for some $\varkappa=\varkappa(\partial \Omega)>1$. In fact, by choosing the constant $\mu$ in (6.82) sufficiently close to 1 , matters can be arranged so that the family $\left\{\varkappa Q_{i}\right\}_{i \in \mathcal{I}}$ has finite overlap. Keeping this in mind and availing ourselves of the fact that $\rho(X) \sim l_{i}$ uniformly for $X \in Q_{i}, i \in \mathcal{I}$, for each multi-index $\gamma$ of length $m$ we may then estimate:

$$
\begin{aligned}
& \int_{\Omega}\left|D^{\gamma} \mathcal{E} \dot{f}(X)\right|^{p} \rho(X)^{p(1-s)-1} d X \\
& \quad \leq C \sum_{j \in \mathbb{Z}} \sum_{i \in I_{j}} 2^{-j p(1-s)-j} \int_{Q_{i}}\left|D^{\gamma} \mathcal{E} \dot{f}(X)\right|^{p} d X \\
& \quad \leq C \sum_{j \in \mathbb{Z}} \sum_{i \in I_{j}} \sum_{|\tau| \leq m-1} 2^{j p(m-1+s-|\tau|)+j(n-1)} \iint_{Y, Z \in \partial \Omega \cap \varkappa Q_{i}}\left|R_{\tau}(Y, Z)\right|^{p} d \sigma_{Y} d \sigma_{Z} \\
& \quad \leq C \sum_{j \in \mathbb{Z}}^{\infty} \sum_{|\gamma-Z| \leq m-1<2^{-j}} 2^{j p(m-1+s-|\tau|)+j(n-1)} \int_{\substack{Y, Z \in \partial \Omega \\
|Y-Z|<\varkappa 2^{-j}}}\left|R_{\tau}(Y, Z)\right|^{p} d \sigma_{Y} d \sigma_{Z} \\
& \quad \leq C \sum_{|\tau| \leq m-1} \int_{0}^{\infty} \frac{r_{\tau}(t)^{p}}{t^{p(m-1+s-|\tau|)+n-1}} d t
\end{aligned}
$$




$$
\leq C\|\dot{f}\|_{\dot{B}_{p}^{m-1+s}(\partial \Omega)}^{p},
$$

where in the last step we have used (6.49). This proves that the operator (6.54) is well-defined and bounded.

Step III: The right-invertibility property. We shall now show that the operator (6.76) is a rightinverse for the trace operator (6.55), i.e., whenever $\dot{f}=\left\{f_{\gamma}\right\}_{|\gamma| \leq m-1} \in \dot{B}_{p}^{m-1+s}(\partial \Omega)$, there holds

$$
f_{\gamma}=i^{|\gamma|} \operatorname{Tr}\left[D^{\gamma} \mathcal{E} \dot{f}\right]
$$

for every multi-index $\gamma$ of length $\leq m-1$. To this end, for $|\gamma| \leq m-1$ we write

$$
D^{\gamma} \mathcal{E} \dot{f}(X)-\mathcal{E}_{\gamma} \dot{f}(X)=\sum_{\substack{\alpha+\beta=\gamma \\|\alpha| \geq 1}} \frac{\gamma !}{\alpha ! \beta !} \int_{\partial \Omega} D_{X}^{\alpha} \mathcal{K}(X, Y)\left(P_{\beta}(X, Y)-P_{\beta}(X, Z)\right) d \sigma_{Y}
$$

where

$$
\mathcal{E}_{\gamma} \dot{f}(X):=\int_{\partial \Omega} \mathcal{K}(X, Y) P_{\gamma}(X, Y) d \sigma_{Y}, \quad X \in \Omega .
$$

Estimating the right-hand side in (6.88) in the same way as we did with the right-hand side of (6.81), we obtain

$$
\begin{aligned}
\int_{\partial \Omega}\left|D^{\gamma} \mathcal{E} \dot{f}(X)-\mathcal{E}_{\gamma} \dot{f}(X)\right|^{p} \rho(X)^{-p s-1} d X & \leq C \sum_{|\tau| \leq m-1} \int_{0}^{\infty} \frac{r_{\tau}(t)^{p}}{t^{p(|\gamma|+s-|\tau|)+n-1}} d t \\
& \leq C\|\dot{f}\|_{\dot{B}_{p}^{m-1+s}(\partial \Omega)}^{p}
\end{aligned}
$$

In a similar fashion, we check that

$$
\begin{aligned}
\int_{\partial \Omega}\left|\nabla\left(D^{\gamma} \mathcal{E} \dot{f}(X)-\mathcal{E}_{\gamma} \dot{f}(X)\right)\right|^{p} \rho(X)^{p-p s-1} d X \\
\leq C \sum_{|\tau| \leq m-1} \int_{0}^{\infty} \frac{r_{\tau}(t)^{p}}{t^{p(|\gamma|+s-|\tau|)+n-1}} d t \leq C\|\dot{f}\|_{\dot{B}_{p}^{m-1+s}(\partial \Omega)}^{p}
\end{aligned}
$$

The two last inequalities imply $D^{\gamma} \mathcal{E} \dot{f}-\mathcal{E} \dot{f} \in V_{p}^{1, a}(\Omega)$ and, therefore,

$$
\operatorname{Tr}\left(D^{\gamma} \mathcal{E} \dot{f}-\mathcal{E}_{\gamma} \dot{f}\right)=0 .
$$

Going further, let us set

$$
E g(X):=\int_{\partial \Omega} \mathcal{K}(X, Y) g(Y) d \sigma_{Y}, \quad X \in \Omega
$$

A simpler version of the reasoning in Step II yields that $E$ maps $B_{p}^{s}(\partial \Omega)$ boundedly into $W_{p}^{1, a}(\Omega)$. Also, a standard argument based on the Poisson kernel-like behavior of $\mathcal{K}(X, Y)$ shows that $\operatorname{Tr} E g=$ $g$ for each $g \in B_{p}^{s}(\partial \Omega)$. 
Based on the definition (6.50) and (6.89) we have

$$
\begin{aligned}
\mid \mathcal{E}_{\gamma} \dot{f}(X) & -\left.E f_{\gamma}(X)\right|^{p}+\rho(X)^{p}\left|\nabla\left(\mathcal{E}_{\gamma} \dot{f}(X)-E f_{\gamma}(X)\right)\right|^{p} \\
& \leq C \sum_{\substack{|\beta| \leq m-1-|\gamma| \\
|\beta| \geq 1}} \rho(X)^{p|\beta|} f_{\Gamma_{\rho(X)}}\left|f_{\gamma+\beta}(Y)\right|^{p} d \sigma_{Y} .
\end{aligned}
$$

Consequently, for an arbitrary Whitney cube $Q_{i}$ we have

$$
\begin{gathered}
\int_{Q_{i}}\left|\mathcal{E}_{\gamma} \dot{f}(X)-E f_{\gamma}(X)\right|^{p} \rho(X)^{-p s-1} d X+\int_{B_{\delta}}\left|\nabla\left(\mathcal{E}_{\gamma} \dot{f}(X)-E f_{\gamma}(X)\right)\right|^{p} \rho(X)^{p-p s-1} d X \\
\leq C \sum_{\substack{|\beta| \leq m-1-|\gamma| \\
|\beta| \geq 1}} l_{i}^{p(|\beta|-s)} \int_{\partial \Omega \cap \varkappa Q_{i}}\left|f_{\gamma+\beta}(Y)\right|^{p} d \sigma_{Y} .
\end{gathered}
$$

Summing over all Whitney cubes we find

$$
\left\|\mathcal{E}_{\gamma} \dot{f}-E f_{\gamma}\right\|_{V_{p}^{1, a}(\Omega)} \leq C \sum_{|\alpha| \leq m-1}\left\|f_{\alpha}\right\|_{L_{p}(\partial \Omega)}
$$

which implies

$$
\operatorname{Tr}\left(\mathcal{E}_{\gamma} \dot{f}-E f_{\gamma}\right)=0 .
$$

Finally, combining (6.97), (6.92), and $\operatorname{Tr} E f_{\gamma}=f_{\gamma}$, we arrive at (6.87).

Step IV: The kernel of the trace. We now turn to the task of identifying the null-space of the trace operator (6.55)-(6.56). For each $k \in \mathbb{N}_{0}$ we denote by $\mathcal{P}_{k}$ the collection of all vector-valued, complex coefficient polynomials of degree $\leq k$ (and agree that $\mathcal{P}_{k}=0$ whenever $k$ is a negative integer). The claim we make at this stage is that the null-space of the operator

$$
W_{p}^{m, a}(\Omega) \ni \mathcal{W} \mapsto\left\{\operatorname{Tr}\left[D^{\gamma} \mathcal{W}\right]\right\}_{|\gamma|=m-1} \in B_{p}^{s}(\partial \Omega)
$$

is given by

$$
\mathcal{P}_{m-2}+V_{p}^{m, a}(\Omega)
$$

The fact that the null-space of the trace operator (6.55)-(6.56) is $V_{p}^{m, a}(\Omega)$ follows readily from this.

That (6.99) is included in the null-space of the operator (6.98) is obvious. The opposite inclusion amounts to showing that if $\mathcal{W} \in W_{p}^{m, a}(\Omega)$ is such that $\operatorname{Tr}\left[D^{\gamma} \mathcal{W}\right]=0$ for all multi-indices $\gamma$ with $|\gamma|=m-1$, then there exists $P_{m-2} \in \mathcal{P}_{m-2}$ with the property that $\mathcal{W}-P_{m-2} \in V_{p}^{m, a}(\Omega)$. To this end, we note that the case $m=1$ is a consequence of (5.19) and consider next the case $m=2$, i.e. when

$$
\mathcal{W} \in W_{p}^{2, a}(\Omega), \quad \operatorname{Tr}[\nabla \mathcal{W}]=0 \quad \text { on } \partial \Omega
$$

Assume that $\left\{\mathcal{W}_{j}\right\}_{j \geq 1}$ is a sequence of smooth in $\bar{\Omega}$ (even polynomial) vector-valued functions approximating $\mathcal{W}$ in $W_{p}^{2, a}(\Omega)$. In particular, 


$$
\operatorname{Tr}\left[\nabla \mathcal{W}_{j}\right] \rightarrow 0 \quad \text { in } \quad L_{p}(\partial \Omega) \quad \text { as } j \rightarrow \infty
$$

If in a neighborhood of a point on $\partial \Omega$ the domain $\Omega$ is given by $\left\{X: X_{n}>\varphi\left(X^{\prime}\right)\right\}$ for some Lipschitz function $\varphi$, the following chain rule holds for the gradient of the function $w_{j}: B^{\prime} \ni X^{\prime} \mapsto$ $\mathcal{W}_{j}\left(X^{\prime}, \varphi\left(X^{\prime}\right)\right)$, where $B^{\prime}$ is a $(n-1)$-dimensional ball:

$$
\nabla w_{j}\left(X^{\prime}\right)=\left.\left(\nabla_{Y^{\prime}} \mathcal{W}_{j}\left(Y^{\prime}, \varphi\left(X^{\prime}\right)\right)\right)\right|_{Y^{\prime}=X^{\prime}}+\left.\left(\frac{\partial}{\partial Y_{n}} \mathcal{W}_{j}\left(X^{\prime}, Y_{n}\right)\right)\right|_{Y_{n}=\varphi\left(X^{\prime}\right)} \nabla \varphi\left(X^{\prime}\right)
$$

Since the sequence $\left\{w_{j}\right\}_{j \geq 1}$ is bounded in $L_{p}\left(B^{\prime}\right)$ and $\nabla w_{j} \rightarrow 0$ in $L_{p}\left(B^{\prime}\right)$, it follows that there exists a subsequence $\left\{j_{i}\right\}_{i}$ such that $w_{j_{i}} \rightarrow$ const in $L_{p}\left(B^{\prime}\right)$ (see Theorem 1.1.12/2 in [31]). Hence, $\operatorname{Tr} \mathcal{W}=P_{0}=$ const on $\partial \Omega$. In view of $\operatorname{Tr}\left[\mathcal{W}-P_{0}\right]=0$ and $\operatorname{Tr}[\nabla \mathcal{W}]=0$, we may conclude that $\mathcal{W}-P_{0} \in V_{p}^{2, a}(\Omega)$ by Hardy's inequality.

The general case follows in an inductive fashion, by reasoning as before with $D^{\alpha} \mathcal{W}$ with $|\alpha|=$ $m-2$ in place of $\mathcal{W}$.

We now present a short proof of (1.8), based on Proposition 6.5.

Proposition 6.8 Assume that $1<p<\infty, s \in(0,1)$ and $m \in \mathbb{N}$. Then

$$
\|\dot{f}\|_{\dot{B}_{p}^{m-1+s}(\partial \Omega)} \sim \sum_{|\alpha| \leq m-1}\left\|f_{\alpha}\right\|_{B_{p}^{s}(\partial \Omega)},
$$

uniformly for $\dot{f}=\left\{f_{\alpha}\right\}_{|\alpha| \leq m-1} \in \dot{B}_{p}^{m-1+s}(\partial \Omega)$. As a consequence, (1.8) holds.

Proof. The left-pointing inequality in (6.103) is implicit in (6.69). As for the opposite one, let $\dot{f}=\left\{f_{\alpha}\right\}_{|\alpha| \leq m-1} \in \dot{B}_{p}^{m-1+s}(\partial \Omega)$ and, with $a:=1-s-1 / p$, consider $\mathcal{U}:=\mathcal{E}(\dot{f}) \in W_{p}^{m, a}(\Omega)$. Then Lemma 6.4 implies that, for each multi-index $\alpha$ of length $\leq m-1$, the function $f_{\alpha}=i^{|\alpha|} \operatorname{Tr}\left[D^{\alpha} \mathcal{U}\right]$ belongs to $B_{p}^{s}(\partial \Omega)$, plus a naturally accompanying norm estimate. This concludes the proof of (6.103). Finally, the last claim in the proposition is a consequence of (6.103), (6.44) and the fact that the operator (6.55)-(6.56) is onto.

We include one more equivalent characterization of the space $\dot{B}_{p}^{m-1+s}(\partial \Omega)$, in the spirit of work in [1], 42], [50]. To state it, recall that $\left\{e_{j}\right\}_{j}$ is the canonical orthonormal basis in $\mathbb{R}^{n}$.

Proposition 6.9 Assume that $1<p<\infty, s \in(0,1)$ and $m \in \mathbb{N}$. Then

$$
\left\{f_{\alpha}\right\}_{|\alpha| \leq m-1} \in \dot{B}_{p}^{m-1+s}(\partial \Omega) \Longleftrightarrow\left\{\begin{array}{c}
f_{\alpha} \in B_{p}^{s}(\partial \Omega), \quad \forall \alpha:|\alpha| \leq m-1 \\
\text { and } \\
\left(\nu_{j} \partial_{k}-\nu_{k} \partial_{j}\right) f_{\alpha}=\nu_{j} f_{\alpha+e_{k}}-\nu_{k} f_{\alpha+e_{j}} \\
\forall \alpha:|\alpha| \leq m-2, \quad \forall j, k \in\{1, \ldots, n\} .
\end{array}\right.
$$

Proof. The left-to-right implication is a consequence of (6.103) and of the fact that (6.73) holds for some $\mathcal{U} \in W_{p}^{m, a}(\Omega)$ (cf. Proposition 6.5). As for the opposite implication, we proceed as in the proof of Proposition 6.5 and estimate (6.48) based on the identities (6.59) and knowledge that $f_{\alpha}$ belongs to $B_{p}^{s}(\partial \Omega)$ for each $\alpha$ of length $\leq m-1$. 
We close this section with two remarks on the nature of the space $\dot{B}_{p}^{m-1+s}(\partial \Omega)$. First, we claim that the assignment

$$
\dot{B}_{p}^{m-1+s}(\partial \Omega) \ni \dot{f}=\left\{f_{\alpha}\right\}_{|\alpha| \leq m-1} \mapsto\left\{i^{k} \sum_{|\alpha|=k} \frac{k !}{\alpha !} \nu^{\alpha} f_{\alpha}\right\}_{0 \leq k \leq m-1} \in L_{p}(\partial \Omega)
$$

is one-to-one. This is readily justified with the help of the identity

$$
D^{\alpha}=i^{-|\alpha|} \nu^{\alpha} \frac{\partial^{|\alpha|}}{\partial \nu^{|\alpha|}}+\sum_{|\beta|=|\alpha|-1} \sum_{j, k=1}^{n} p_{\alpha, \beta, j, k}(\nu) \frac{\partial}{\partial \tau_{j k}} D^{\beta}
$$

where $\partial / \partial \tau_{j k}:=\nu_{j} \partial / \partial x_{k}-\nu_{k} \partial / \partial x_{j}$ and the $p_{\alpha, \beta, j, k}$ 's are polynomial functions. Indeed, let $\dot{f} \in \dot{B}_{p}^{m-1+s}(\partial \Omega)$ be mapped to zero by the assignment (6.105) and consider $\mathcal{U}:=\mathcal{E}(\dot{f}) \in W_{p}^{m, a}(\Omega)$. Then $f_{\alpha}=i^{|\alpha|} \operatorname{Tr}\left[D^{\alpha} \mathcal{U}\right]$ on $\partial \Omega$ for each $\alpha$ with $|\alpha| \leq m-1$ and, granted the current hypotheses, $\partial^{k} \mathcal{U} / \partial \nu^{k}=0$ for $k=0,1, \ldots, m-1$. Consequently, (6.106) and induction on $|\alpha|$ yield that $\operatorname{Tr}\left[D^{\alpha} \mathcal{U}\right]=0$ on $\partial \Omega$ for each $\alpha$ with $|\alpha| \leq m-1$. Thus, $f_{\alpha}=0$ for each $\alpha$ with $|\alpha| \leq m-1$, as desired.

The elementary identity [6.106) can be proved by writing

$$
\begin{aligned}
i^{|\alpha|} D^{\alpha} & =\prod_{j=1}^{n}\left(\frac{\partial}{\partial x_{j}}\right)^{\alpha_{j}} \\
& =\left.\prod_{j=1}^{n}\left[\sum_{k=1}^{n} \xi_{k}\left(\xi_{k} \frac{\partial}{\partial x_{j}}-\xi_{j} \frac{\partial}{\partial x_{k}}\right)+\sum_{k=1}^{n} \xi_{j} \xi_{k} \frac{\partial}{\partial x_{k}}\right]^{\alpha_{j}}\right|_{\xi=\nu} \\
& =\left.\prod_{j=1}^{n}\left[\sum_{l=0}^{\alpha_{j}} \frac{\alpha_{j} !}{l !\left(\alpha_{j}-l\right) !}\left(\sum_{k=1}^{n} \xi_{k}\left(\xi_{k} \frac{\partial}{\partial x_{j}}-\xi_{j} \frac{\partial}{\partial x_{k}}\right)\right)^{\alpha_{j}-l} \nu_{j}^{l} \frac{\partial^{l}}{\partial \nu^{l}}\right]\right|_{\xi=\nu} \\
& =\left.\prod_{j=1}^{n}\left[\nu_{j}^{\alpha_{j}} \frac{\partial^{\alpha_{j}}}{\partial \nu^{\alpha_{j}}}+\sum_{l=0}^{\alpha_{j}-1} \frac{\alpha_{j} !}{l !\left(\alpha_{j}-l\right) !}\left(\sum_{k=1}^{n} \xi_{k}\left(\xi_{k} \frac{\partial}{\partial x_{j}}-\xi_{j} \frac{\partial}{\partial x_{k}}\right)\right)^{\alpha_{j}-l} \nu_{j}^{l} \frac{\partial^{l}}{\partial \nu^{l}}\right]\right|_{\xi=\nu}
\end{aligned}
$$

and noticing that $\prod_{j=1}^{n} \nu_{j}^{\alpha_{j}} \partial^{\alpha_{j}} / \partial \nu^{\alpha_{j}}=\nu^{\alpha} \partial^{|\alpha|} / \partial \nu^{|\alpha|}$, whereas $\left.\left(\xi_{k} \partial / \partial x_{j}-\xi_{j} \partial / \partial x_{k}\right)\right|_{\xi=\nu}=-\partial / \partial \tau_{j k}$.

Our second remark concerns the image of the mapping (6.105) in the case when $\partial \Omega$ is sufficiently smooth. More precisely, assume that $\partial \Omega \in C^{m-1,1}$ and, for $0 \leq k \leq m-1$, the space $B_{p}^{m-1-k+s}(\partial \Omega)$ is defined starting from $B_{p}^{m-1-k+s}\left(\mathbb{R}^{n-1}\right)$ and then transporting this space to $\partial \Omega$ via a smooth partition of unity argument and locally flattening the boundary (alternatively, $B_{p}^{m-1-k+s}(\partial \Omega$ ) is the image of the trace operator acting from $B_{p}^{m-1-k+s+1 / p}\left(\mathbb{R}^{n}\right)$ ). We claim that

$$
\partial \Omega \in C^{m-1,1} \Longrightarrow \text { the image of the mapping (6.105] is } \oplus_{k=0}^{m-1} B_{p}^{m-1-k+s}(\partial \Omega) \text {. }
$$

Indeed, granted that $\partial \Omega \in C^{m-1,1}$, it follows from (6.104) that $f_{\alpha} \in B_{p}^{m-1-|\alpha|+s}(\partial \Omega)$ for each $\alpha$ with $|\alpha| \leq m-1$ and, hence, $g_{k}:=i^{k} \sum_{|\alpha|=k} \frac{k !}{\alpha !} \nu^{\alpha} f_{\alpha} \in B_{p}^{m-1-k+s}(\partial \Omega)$ for each $k \in\{0, \ldots, m-1\}$.

Conversely, given a family $\left\{g_{k}\right\}_{0 \leq k \leq m-1} \in \oplus_{k=0}^{m-1} B_{p}^{m-1-k+s}(\partial \Omega)$, we claim that there exists $\dot{f}=\left\{f_{\alpha}\right\}_{|\alpha| \leq m-1} \in \dot{B}_{p}^{m-1+s}(\partial \Omega)$ such that $g_{k}=i^{k} \sum_{|\alpha|=k} \frac{k !}{\alpha !} \nu^{\alpha} f_{\alpha}$ for each $k \in\{0, \ldots, m-1\}$. One 
way to see this is to start with $\mathcal{U} \in B_{p}^{m-1+s+1 / p}(\Omega)$ solution of $\Delta^{m} \mathcal{U}=0$ in $\Omega, \partial^{k} \mathcal{U} / \partial \nu^{k}=g_{k} \in$ $B_{p}^{m-1-k+s}(\partial \Omega), 0 \leq k \leq m-1$ (a system which satisfies the Shapiro-Lopatinskij condition) and then define the $f_{\alpha}$ 's as in (6.73).

\subsection{Proof of the main result and further comments}

Theorem 1.1 is a particular case of the next theorem concerning the unique solvability of the Dirichlet problem in $W_{p}^{m, a}(\Omega)$.

Theorem 6.10 Let all assumptions of Theorem 6.3 be satisfied. Also let $\mathcal{F} \in V_{p}^{-m, a}(\Omega)$. Then the Dirichlet problem

$$
\left\{\begin{array}{l}
\mathcal{A}\left(X, D_{X}\right) \mathcal{U}=\mathcal{F} \quad \text { in } \Omega, \\
\frac{\partial^{k} \mathcal{U}}{\partial \nu^{k}}=g_{k} \quad \text { on } \partial \Omega, \quad 0 \leq k \leq m-1,
\end{array}\right.
$$

has a solution $\mathcal{U} \in W_{p}^{m, a}(\Omega)$ if and only if (1.9) is satisfied. In this latter case, the solution is unique and satisfies

$$
\|\mathcal{U}\|_{W_{p}^{m, a}(\Omega)} \leq C \sum_{|\alpha| \leq m-1}\left\|f_{\alpha}\right\|_{B_{p}^{s}(\partial \Omega)}+C\|\mathcal{F}\|_{V_{p}^{-m, a}(\Omega)}
$$

Proof. It is clear from definitions that the operator

$$
\mathcal{A}\left(X, D_{X}\right): W_{p}^{m, a}(\Omega) \longrightarrow V_{p}^{-m, a}(\Omega)
$$

is well-defined and bounded. Thus, granted that we seek solutions for (6.109) in the space $W_{p}^{m, a}(\Omega)$, the membership of $\mathcal{F}$ to $V_{p}^{-m, a}(\Omega)$, as well as the fact that the $g_{k}$ 's satisfy (1.9), are necessary conditions for the solvability of (6.109).

Conversely, let $\dot{f}=\left\{f_{\alpha}\right\}_{|\alpha| \leq m-1} \in \dot{B}_{p}^{m-1+s}(\partial \Omega)$ be such that (1.9) holds and, with $\mathcal{E}$ denoting the extension operator from Proposition 6.5, seek a solution for (6.109) in the form $\mathcal{U}=\mathcal{E}(\dot{f})+\mathcal{W}$. where $\mathcal{W} \in V_{p}^{m, a}(\Omega)$ solves

$$
\left\{\begin{array}{l}
\mathcal{A}\left(X, D_{X}\right) \mathcal{W}=\mathcal{F}-\mathcal{A}\left(X, D_{X}\right)(\mathcal{E}(\dot{f})) \quad \text { in } \Omega \\
\operatorname{Tr}\left[D^{\gamma} \mathcal{W}\right]=0 \quad \text { on } \partial \Omega, \forall \gamma:|\gamma| \leq m-1 .
\end{array}\right.
$$

Since the boundary conditions in (6.112) are automatically satisfied if $\mathcal{W} \in V_{p}^{m, a}(\Omega)$, the solvability of (6.112) is a direct consequence of Theorem 6.3. As for uniqueness, assume that $\mathcal{U} \in W_{p}^{m, a}(\Omega)$ solves (6.109) with $\mathcal{F}=0$ and $g_{k}=0,0 \leq k \leq m-1$. From the fact that (6.105) is one-to-one, we infer that $\operatorname{Tr}\left[D^{\gamma} \mathcal{U}\right]=0$ on $\partial \Omega$ for all $\gamma$ with $|\gamma| \leq m-1$. Then, by Proposition 6.5, $\mathcal{U} \in V_{p}^{m, a}(\Omega)$ is a null-solution of $\mathcal{A}\left(X, D_{X}\right)$. In turn, Theorem 6.3 gives that $\mathcal{U}=0$, proving uniqueness for (6.109). Finally, (6.110) is a consequence of the results in $\S 6.4$.

We conclude this section with a couple of comments, the first of which regards the effect of the presence of lower order terms. More specifically, assume that

$$
\mathcal{A}\left(X, D_{X}\right) \mathcal{U}:=\sum_{0 \leq|\alpha|,|\beta| \leq m} D^{\alpha}\left(\mathcal{A}_{\alpha \beta}(X) D^{\beta} \mathcal{U}\right), \quad X \in \Omega
$$


where the top part of $\mathcal{A}\left(X, D_{X}\right)$ satisfies the hypotheses made in Theorem 1.1 and the lower order terms are bounded. Then the Dirichlet problem (6.109) is Fredholm solvable, of index zero, in the sense that a solution $\mathcal{U} \in W_{p}^{m, a}(\Omega)$ exists if and only if the data $\mathcal{F},\left\{g_{k}\right\}_{0 \leq k \leq m-1}$ satisfy finitely many linear conditions, whose number matches the dimension of the space of null-solutions for (6.109). Furthermore, the estimate

$$
\|\mathcal{U}\|_{W_{p}^{m, a}(\Omega)} \leq C\left(\sum_{|\alpha| \leq m-1}\|\mathcal{F}\|_{V_{p}^{-m, a}(\Omega)}+\left\|f_{\alpha}\right\|_{B_{p}^{s}(\partial \Omega)}+\|\mathcal{U}\|_{L_{p}(\Omega)}\right)
$$

holds for any solution $\mathcal{U} \in W_{p}^{m, a}(\Omega)$ of (6.109).

Indeed, the operator

$$
\mathcal{A}: V_{p}^{m, a}(\Omega) \longrightarrow V_{p}^{-m, a}(\Omega)
$$

is Fredholm with index zero, as can be seen by decomposing $\mathcal{A}=\mathcal{A}+(\mathcal{A}-\mathcal{A})$ where $\mathcal{A}:=$ $\sum_{|\alpha|=|\beta|=m} D^{\alpha} \mathcal{A}_{\alpha \beta} D^{\beta}$, and then invoking Theorem 6.3. Now, it can be shown that the problem (6.109) is solvable if and only if $\mathcal{F}-\mathcal{A}\left(X, D_{X}\right) \mathcal{E} \dot{f} \in \operatorname{Im} \mathcal{A}$, the image of the operator (6.115). Thus, if $T\left(\mathcal{F},\left\{g_{k}\right\}_{0 \leq k \leq m-1}\right):=\mathcal{F}-\mathcal{A}\left(X, D_{X}\right) \mathcal{E} \dot{f}$, this membership entails $\left(\mathcal{F},\left\{g_{k}\right\}_{0 \leq k \leq m-1}\right) \in T^{-1}(\operatorname{Im} \mathcal{A})$. Note that $T$ maps the space of data onto $V_{p}^{-m, a}(\Omega)$, hence the number of linearly independent compatibility conditions the data should satisfy is

$$
\operatorname{codim} T^{-1}(\operatorname{Im} \mathcal{A})=\operatorname{codim}(\operatorname{Im} \mathcal{A})
$$

On the other hand, from by Proposition 6.5 and the fact that (6.105) is one-to-one we infer that the space of null-solutions for (6.109) is precisely ker $\mathcal{A}$, the kernel of the operator (6.115). Since, as already pointed out, this operator has index zero, it follows that the problem (6.109) has index zero. Finally, (6.114) follows from what we have proved so far via a standard reasoning as in [23].

Our last comment regards the statement of the Dirichlet problem (1.2) with data

$$
\partial^{k} \mathcal{U} / \partial \nu^{k}=g_{k} \in B_{p}^{m-1-k+s}(\partial \Omega), \quad k=0,1, \ldots, m-1,
$$

where $B_{p}^{m-1-k+s}(\partial \Omega)$ is defined here as the range of $\operatorname{Tr}$ acting from $B_{p}^{m-1-k+s+1 / p}\left(\mathbb{R}^{n}\right)$. If $\partial \Omega$ is smooth $\left(C^{1,1}\right.$ will do) this problem is, certainly, well-posed. Let us illustrate some features of this particular formulation as the smoothness of $\partial \Omega$ deteriorates.

Suppose we are looking for the solution $\mathcal{U} \in W_{2}^{2}(\Omega)$ of the Dirichlet problem for the biharmonic operator

$$
\left\{\begin{array}{l}
\Delta^{2} \mathcal{U}=0 \quad \text { in } \Omega, \\
\operatorname{Tr} \mathcal{U}=g_{1} \quad \text { on } \partial \Omega \\
\langle\nu, \operatorname{Tr}[\nabla \mathcal{U}]\rangle=g_{2} \quad \text { on } \partial \Omega .
\end{array}\right.
$$

The simplest class of data $\left(g_{1}, g_{2}\right)$ would be, of course, $B_{2}^{3 / 2}(\partial \Omega) \times B_{2}^{1 / 2}(\partial \Omega)$, where $B_{2}^{3 / 2}(\partial \Omega)$ and $B_{2}^{1 / 2}(\partial \Omega)$ are the spaces of traces on $\partial \Omega$ for functions in $W_{2}^{2}(\Omega)$ and $W_{2}^{1}(\Omega)$, respectively. However, this formulation has several serious drawbacks.

The first one is that the mapping

$$
W_{2}^{2}(\Omega) \ni \mathcal{U} \rightarrow\langle\nu, \operatorname{Tr}[\nabla \mathcal{U}]\rangle \in B_{2}^{1 / 2}(\partial \Omega)
$$


is generally unbounded. In fact, by choosing $\mathcal{U}$ to be a linear function we see that the continuity of (6.119) implies $\nu \in B_{2}^{1 / 2}(\partial \Omega)$ which is not necessarily the case for a Lipschitz domain, even for such a simple one as the square $S=[0,1]^{2}$.

The same problem fails to have a solution in the class in $W_{2}^{2}(\Omega)$ when when $\left(g_{1}, g_{2}\right)$ is an arbitrary pair in $B_{2}^{3 / 2}(\partial \Omega) \times B_{2}^{1 / 2}(\partial \Omega)$. Indeed, consider the problem (6.118) for $\Omega=S$ and the data $g_{1}=0$ and $g_{2}=1$. It is standard (see Theorem 7.2.4 in [29] and Sect.7.1 in [30]) that the main term of the asymptotics near the origin of any solution $\mathcal{U}$ in $W_{2}^{1}(S)$ is given in polar coordinates $(r, \omega)$ by

$$
\frac{2 r}{\pi+2}\left(\left(\omega-\frac{\pi}{2}\right) \sin \omega-\omega \cos \omega\right)
$$

Since this function does not belong to $W_{2}^{2}(S)$, there is no solution of problem (6.118) in this space.

\section{References}

[1] V.Adolfsson and J.Pipher, The inhomogeneous Dirichlet problem for $\Delta^{2}$ in Lipschitz domains, J. Funct. Anal., 159 (1998), no. 1, 137-190.

[2] S. Agmon, Multiple layer potentials and the Dirichlet problem for higher order elliptic equations in the plane. I, Comm. Pure Appl. Math., 10 (1957) 179-239.

[3] S. Agmon, The $L^{p}$ approach to the Dirichlet problem. I. Regularity theorems, Ann. Scuola Norm. Sup. Pisa (3), 13 (1959) 405-448.

[4] S. Agmon, A. Douglis and L. Nirenberg, Estimates near the boundary for solutions of elliptic partial differential equations satisfying general boundary conditions. II, Comm. Pure Appl. Math., 17 (1964) $35-92$.

[5] P. Auscher and M. Qafsaoui, Observations on estimates for divergence elliptic equations with VMO coefficients, Boll. Unione Mat. Ital. Sez. B Artic. Ric. Mat., 5 (2002), 487-509.

[6] S. Byun, Elliptic equations with BMO coefficients in Lipschitz domains, Trans. Amer. Math. Soc., 357 (2005) 1025-1046.

[7] L.A. Caffarelli and I. Peral, On estimates for elliptic equations in divergence form, Comm. Pure Appl. Math., 51, (1998), 1-21.

[8] A.P. Calderón and A.Zygmund, On the existence of certain singular integrals, Acta Math., 88, (1952), 85-139.

[9] A.P. Calderón and A.Zygmund, On singular integrals with variable kernels, Applicable Anal., 7 $(1977 / 78)$, no. 3, 221-238.

[10] F. Chiarenza, M. Frasca and P. Longo, Interior estimates for nondivergence elliptic equations with discontinuous coefficients, Ricerche Mat., 40, (1991), 149-168.

[11] F. Chiarenza, M. Frasca and P. Longo, Solvability of the Dirichlet problem for nondivergence elliptic equations with VMO coefficients, Trans. Amer. Math. Soc., 336, (1993), 841-853.

[12] R. Coifman, R. Rochberg, and G. Weiss, Factorization theorems for Hardy spaces in several variables, Ann. Math., 103 (1976), 611-635. 
[13] B. E. Dahlberg, Poisson semigroups and singular integrals, Proc. Amer. Math. Soc., 97 (1986), no. 1, 41-48.

[14] B. E. Dahlberg and C. E. Kenig, Hardy spaces and the Neumann problem in $L^{p}$ for Laplace's equation in Lipschitz domains, Ann. of Math., 125 (1987), no. 3, 437-465.

[15] B. E. Dahlberg, C. E. Kenig, J. Pipher and G.C. Verchota, Area integral estimates for higher order elliptic equations and systems, Ann. Inst. Fourier (Grenoble), 47 (1997), no. 5, 1425-1461.

[16] E. Fabes, Layer potential methods for boundary value problems on Lipschitz domains, pp. 55-80 in Potential Theory - Surveys and Problems Lecture Notes in Math., Vol.1344, Springer, Berlin, 1988.

[17] C. Fefferman and E.M. Stein, $H^{p}$ spaces of several variables, Acta Math., 129 (1972), no. 3-4, 137-193.

[18] E. Gagliardo, Caratterizzazioni delle tracce sulla frontiera relative ad alcune classi di funzioni in $n$ variabili, Rend. Sem. Mat. Univ. Padova, 27 (1957) 284-305.

[19] G. Di Fazio, Estimates for divergence form elliptic equations with discontinuous coefficients, Boll. Un. Mat. Ital A(7), 10, (1996), 409-420.

[20] P. Grisvard, Elliptic Problems in Nonsmooth Domains, Monographs and Studies in Mathematics, Vol. 24, Pitman, Boston, MA, 1985.

[21] D. Guidetti, General linear boundary value problems for elliptic operators with VMO coefficients, Math. Nachr., 237 (2002), 62-88.

[22] S. Hofmann and J.L. Lewis, $L^{2}$ solvability and representation by caloric layer potentials in time-varying domains, Ann. of Math. (2), 144 (1996), no. 2, 349-420.

[23] L. Hörmander, Linear Partial Differential Operators, Springer Verlag, Berlin-New York, 1976.

[24] T. Iwaniec and C. Sbordone, Riesz transforms and elliptic PDEs with VMO coefficients, J. Anal. Math., 74 (1998), 183-212.

[25] D. Jerison and C. Kenig, The inhomogeneous Dirichlet problem in Lipschitz domains, J. Funct. Anal., 130 (1995), 161-219.

[26] A. Jonsson and H. Wallin, Function Spaces on Subsets of $\mathbb{R}^{n}$, University of Umea, Mathematical Reports, Volume 2, Part 1, 1984.

[27] C.E. Kenig, Harmonic analysis techniques for second order elliptic boundary value problems, CBMS Regional Conference Series in Mathematics, Vol. 83, American Mathematical Society, Providence, RI, 1994.

[28] V. Kozlov and V. Maz'ya, Asymptotic formula for solutions to elliptic equations near Lipschitz boundary, to appear in Ann. Mat. Pura ed Appl., (2005).

[29] V.A. Kozlov, V.G. Maz'ya and J. Rossmann, Elliptic Boundary Value Problems in Domains with Point Singularities, AMS, 1997.

[30] V.A. Kozlov, V.G. Maz'ya and J. Rossmann, Spectral Problems Associated with Corner Singularities of Solutions to Elliptic Equations, AMS, 2001.

[31] V. Maz'ya, Sobolev Spaces, Springer Series in Soviet Mathematics. Springer-Verlag, Berlin, 1985.

[32] V. Maz'ya and T.Shaposhnikova, Theory of Multipliers in Spaces of Differentiable Functions, Monographs and Studies in Mathematics Vol.23, Pitman Advanced Publishing Program, Boston, MA, 1985 . 
[33] V. Maz'ya and T. Shaposhnikova, On the regularity of the boundary in the Lp-theory of elliptic boundary value problems. I. (Russian) Partial differential equations, pp. 39-56, Trudy Sem. S.L. Soboleva, No. 2, 80, Akad. Nauk SSSR Sibirsk. Otdel., Inst. Mat., Novosibirsk, 1980.

[34] V. Maz'ya and T. Shaposhnikova, Higher regularity in the classical layer potential theory for Lipschitz domains, Indiana Univ. Math. J., 54 (2005), no. 1, 99-142.

[35] N.G. Meyers, An estimate for the gradient of solutions of second order elliptic divergence equations, Ann. Scuola Norm. Sup. Pisa (3), 17, (1963), 189-206.

[36] C. Miranda, Sulle equazioni ellittiche del secondo ordine di tipo non variazionale, a coefficienti discontinui, Ann. Mat. Pura Appl. (4), 63 (1963), 353-386.

[37] D. Mitrea, M. Mitrea and M. Taylor, Layer Potentials, the Hodge Laplacian, and Global Boundary Problems in Nonsmooth Riemannian Manifolds, Mem. Amer. Math. Soc., Vol. 150, No. 713, 2001.

[38] M. Mitrea and M. Taylor, Potential theory on Lipschitz domains in Riemannian manifolds: SobolevBesov space results and the Poisson problem, J. Funct. Anal. 176 (2000), 1-79.

[39] M. Mitrea and M. Taylor, Sobolev and Besov space estimates for solutions to second order PDE on Lipschitz domains in manifolds with Dini or Hölder continuous metric tensors, to appear in Comm. in PDE (2005).

[40] M. Mitrea and M. Taylor, The Poisson problem in weighted Sobolev spaces on Lipschitz domains, to appear in the Indina Math. Univ. Journal, (2005).

[41] J. Nečas, Les Méthodes Directes en Théorie des Équations Elliptiques, Masson et Cie, Éditeurs, Paris, Academia, Éditeurs, Prague, 1967.

[42] J.Pipher and G.C. Verchota, Dilation invariant estimates and the boundary Gairding inequality for higher order elliptic operators, Ann. of Math. (2), 142 (1995), no. 1, 1-38.

[43] M.A. Ragusa, Local Hölder regularity for solutions of elliptic systems, Duke Math. J., 113, (2002), 385-397.

[44] Z. Shen, The $L^{p}$ Dirichlet problem for elliptic systems on Lipschitz domains, preprint (2004).

[45] V.A. Solonnikov, General boundary value problems for systems elliptic in the sense of A. Douglis and L. Nirenberg. I, (Russian) Izv. Akad. Nauk SSSR, Ser. Mat., 28 (1964), 665-706.

[46] V.A. Solonnikov, General boundary value problems for systems elliptic in the sense of A. Douglis and L. Nirenberg. II, (Rusian) Trudy Mat. Inst. Steklov, Vol. 92 (1966), 233-297.

[47] E.M. Stein, Singular Integrals and Differentiability Properties of Functions, Princeton Mathematical Series, No. 30, Princeton University Press, Princeton, N.J. 1970.

[48] A. Torchinsky, Real Variable Methods in Harmonic Analysis, Academic Press, 1986.

[49] S. V. Uspenskiǔ, Imbedding theorems for classes with weights, Trudy Mat. Inst. Steklov., 60 (1961), 282-303.

[50] G.C. Verchota, The Dirichlet problem for the polyharmonic equation in Lipschitz domains, Indiana Univ. Math. J., 39 (1990), 671-702.

[51] G.C.Verchota, The biharmonic Neumann problem in Lipschitz domains, to appear in Acta Mathematica, (2005).

[52] H. Whitney, Analytic extensions of differentiable functions defined in closed sets, Trans. Amer. Math. Soc., 36 (1934), 63-89. 
Vladimir Maz'ya

Department of Mathematics

Ohio State University

Columbus, OH 43210, USA

e-mail:vlmaz@math.ohio-state.edu

and

Department of Mathematical Sciences

University of Liverpool

Liverpool L69 3BX, UK

Marius Mitrea

Department of Mathematics

University of Missouri at Columbia

Columbia, MO 65211, USA

e-mail:marius@math.missouri.edu

Tatyana Shaposhnikova

Department of Mathematics

Ohio State University

Columbus, OH 43210, USA

e-mail:tasha@math.ohio-state.edu

and

Department of Mathematics

Linköping University

Linköping SE-581 83, Sweden

e-mail:tasha@mai.liu.se 